\title{
DISSERTATION
}

\section{A MODELING TOOLKIT FOR COMPARING AC VS. DC ELECTRICAL DISTRIBUTION EFFICIENCY IN BUILDINGS}

\author{
Submitted by \\ Avpreet Othee \\ Department of Systems Engineering
}

In partial fulfillment of the requirements

For the Degree of Doctor of Philosophy

Colorado State University

Fort Collins, Colorado

Summer 2021

Doctoral Committee:

Advisor: James Cale

Peter Young

Daniel Herber

Gaofeng Jia 
Copyright by Avpreet Othee 2021

All Rights Reserved 


\begin{abstract}
A MODELING TOOLKIT FOR COMPARING AC VS. DC ELECTRICAL DISTRIBUTION EFFICIENCY IN BUILDINGS
\end{abstract}

An increasing proportion of electrical devices in residential and commercial buildings operate from direct current (DC) power sources. In addition, distributed power generation systems such as solar photovoltaic $(\mathrm{PV})$ and energy storage natively produce $\mathrm{DC}$ power. However, traditional power distribution is based on an alternating current (AC) model. Performing the necessary conversions between $\mathrm{AC}$ and $\mathrm{DC}$ power to make $\mathrm{DC}$ devices compatible with $\mathrm{AC}$ distribution results in energy losses. For these reasons, DC distribution may offer energy efficiency advantages in comparison to AC distribution. However, reasonably fast computation and comparison of electrical efficiencies of AC-only, DC-only, and hybrid AC/DC distributions systems is challenging because DC devices are typically (nonlinear) power-electronic converters that produce harmonic content. While detailed time-domain modeling can be used to simulate these harmonics, it is not computationally efficient or practical for many building designers.

To address this need, this research describes a toolkit for computation of harmonic spectra and energy efficiency in mixed AC and DC electrical distribution systems, using a Harmonic Power Flow (HPF) methodology. The toolkit includes a library of two-port linear and nonlinear device models which can be used to construct and simulate an electrical distribution system. This dissertation includes a description of the mathematical theory and framework underlying the toolkit, development and fitting of linear and nonlinear device models, software implementation in Modelica, verification of the toolkit with laboratory measurements, and discussion of ongoing and future work to employ the toolkit to a variety of building designs. 


\section{ACKNOWLEDGEMENTS}

I thank my advisor Dr. James Cale for his technical advice, feedback, and encouragement. I am grateful for the guidance and feedback from the rest of my committee - Dr. Peter Young, Dr. Daniel Herber, and Dr. Gaofeng Jia. I would also like to thank Arthur Santos for his efforts in designing the distribution testbed at the Powerhouse Energy Campus and the countless days he spent in taking all the measurements. I want to thank Steven Frank for his leadership as the principal investigator for the project.

Open source software and the larger open source movement has allowed for immense collaboration among scientists and researchers. The collective outcome of the effors of thousands of contributors has resulted in some of the best software and tools. I am grateful for the OpenModelica community for their efforts in development of the OpenModelica.

Lastly, I thank my family for their support and encouragement. A special thanks to my wife Achint for being by my side throughout the whole journey.

Funding was provided by the DOE Assistant Secretary for Energy Efficiency and Renewable Energy Building Technologies Office Emerging Technologies Program. I am extremely grateful for the financial support. 


\section{TABLE OF CONTENTS}

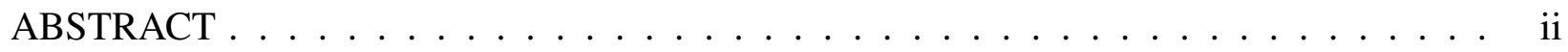

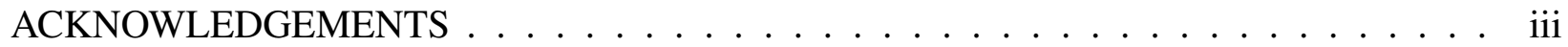

LIST OF TABLES $\ldots \ldots \ldots \ldots \ldots \ldots \ldots \ldots \ldots \ldots$ vi

LIST OF FIGURES . . . . . . . . . . . . . . . . . . . . . . . vii

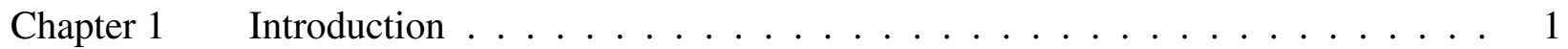

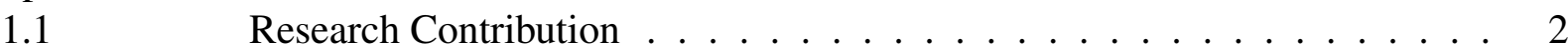

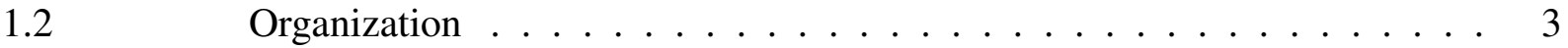

$1.3 \quad$ Nomenclature and Notation $\ldots \ldots \ldots \ldots$

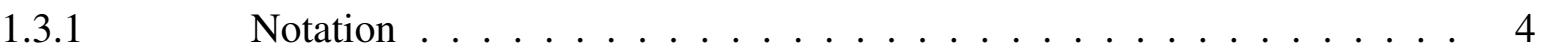

1.3.2 Nomenclature .................. 4

Chapter 2 Background and Related Work . . . . . . . . . . . . . . 6

$2.1 \quad$ Losses in an Electrical Distribution . . . . . . . . . . . . 6

2.2 Effect of Nonlinear Loads on Energy Efficiency . . . . . . . . . . . 8

2.3 Energy Efficiency Modeling Techniques . . . . . . . . . . . . . 10

$2.4 \quad$ Frequency-domain Representation of Non-sinusoidal Signals . . . . . . 11

$2.5 \quad$ Background and Literature Review . . . . . . . . . . . . . 13

2.5.1 Harmonic Power Flow (original formulation) . . . . . . . . . . 13

2.5.2 Harmonic Power Flow Analysis Tools . . . . . . . . . . . . . 16

2.5.3 Load Models . . . . . . . . . . . . . . . . . . 16

Chapter $3 \quad$ Modeling Framework . . . . . . . . . . . . . . . . . . . . . . 19

$3.1 \quad$ Background on Linear AC Analysis . . . . . . . . . . . . . . . 19

$3.2 \quad$ Electrical Network Theory . . . . . . . . . . . . . . . 21

3.2.1 One- and Two-Port Network Theory . . . . . . . . . . . 21

3.2.2 Defining Network Topologies . . . . . . . . . . . . . . . . . . . 24

$3.3 \quad$ Harmonic Power Flow Solution . . . . . . . . . . . . . . 27

Chapter 4 Device Models . . . . . . . . . . . . . . . . . . . . . . 31

$4.1 \quad$ Series and Shunt Impedances $\ldots \ldots \ldots 31$

$4.1 .1 \quad$ Series Impedance $\ldots \ldots \ldots \ldots$. . . . . . . . . . . . . 31

$4.2 \quad$ Constant Power Loads . . . . . . . . . . . . . . . . . 32

$4.3 \quad$ Transformers . . . . . . . . . . . . . . 33

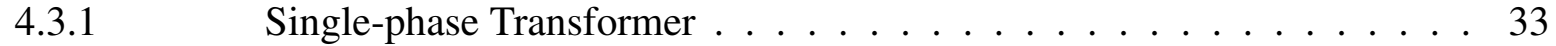

4.3.2 Three-phase Transformers . . . . . . . . . . . . . . . . . 34

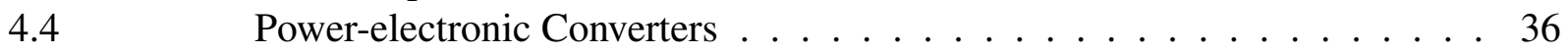

4.4.1 Converter Device-Specific Behavior . . . . . . . . . . . 36

4.4.2 Converter Loss Modeling . . . . . . . . . . . . . . . . . . . 39

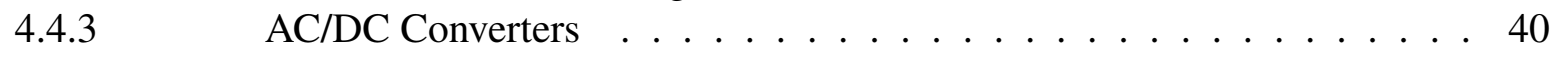

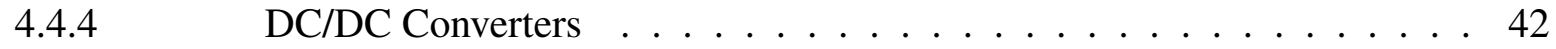


Chapter 5 Application of HPF on an Example Circuit . . . . . . . . . . . . . 43

$5.1 \quad$ Network Modeling . . . . . . . . . . . . . . . . 43

$5.2 \quad$ Solving the Network . . . . . . . . . . . . . . . 47

Chapter $6 \quad$ Software Implementation: Modeling Toolkit . . . . . . . . . . . . . . . . 49

$6.1 \quad$ Modelica: A Systems Modeling Language . . . . . . . . . . . . . . 49

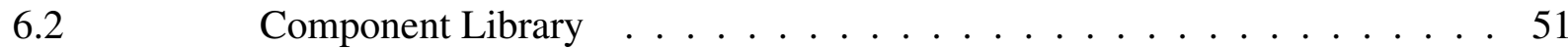

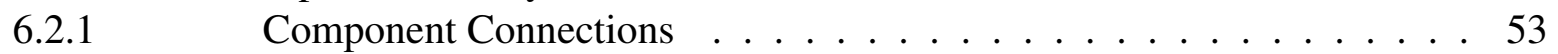

6.2.2 Component Models . . . . . . . . . . . . . . 54

6.3

6.4

6.5

Initialization . . . . . . . . . . . . . . . 57

System Solver . . . . . . . . . . . . . . . . . . . . . . . . . . . . . 58

Solution for Example System . . . . . . . . . . . . . . . 59

Chapter $7 \quad$ Nonlinear Device Behavior Modeling . . . . . . . . . . . . 62

7.0.1 AC/DC Converter Harmonics . . . . . . . . . . . . . . 62

7.1

7.1 .1

7.2

Device-specific Behavioral Function Models . . . . . . . . . . . . 65

Harmonic Magnitude Model . . . . . . . . . . . . . . . . 66

Harmonic Angle Model . . . . . . . . . . . . . . . . . . 66

Parameter Estimation . . . . . . . . . . . . . . . . 67

$7.3 \quad$ Model Validation . . . . . . . . . . . . . . . 69

Chapter 8 Toolkit Validation . . . . . . . . . . . . . . . 72

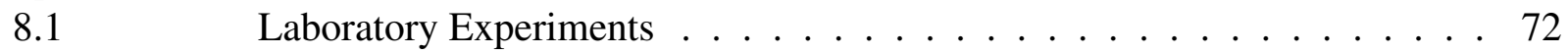

$8.2 \quad$ Comparison of Toolkit Results with Measurements . . . . . . . . . . 76

Chapter 9 Summary and Suggestions for Future Work . . . . . . . . . . . . 83

$9.1 \quad$ Suggestions for Future work . . . . . . . . . . . . . . 85

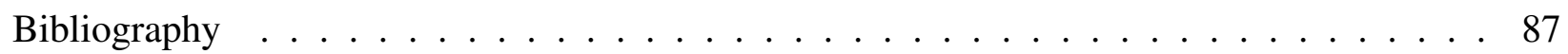

Appendix A Nonlinear load equations . . . . . . . . . . . . . . 95

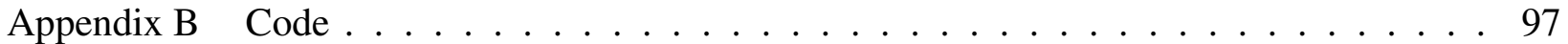

B.1 MATLAB code - Example Circuit . . . . . . . . . . . . . 97

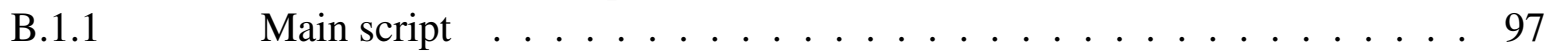

B.1.2 Function handle containing system equations . . . . . . . . . . . . 99

B.1.3 Nonlinear load model . . . . . . . . . . . . . . . . 101

B.2 Modelica Code - Example Circuit . . . . . . . . . . . . . 102

B.2.1 Simulation model . . . . . . . . . . . . . . 102

B.2.2 Nonlinear load . . . . . . . . . . . . . . 102

B.3 BEEAM Library Source Code . . . . . . . . . . . . . . 103

Appendix C Simulation Results . . . . . . . . . . . . . . . . . 104 


\section{LIST OF TABLES}

1.1 Indices and Sets. . . . . . . . . . . . . . . . . 5

1.2 Table of variables . . . . . . . . . . . . . . . . . . 5

1.3 List of functions and parameters. . . . . . . . . . . . . . 5

2.1 Comparison of system efficiencies . . . . . . . . . . . . . . . 9

6.1 Simulation output comparison on nonlinear example problem: Toolkit vs. Scripted

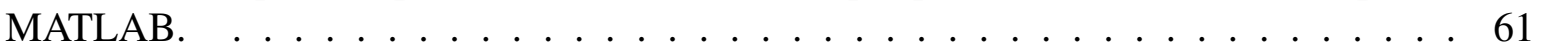

7.1 Devices characterized in the experiment. . . . . . . . . . . . . . 69

8.1 Device configurations for AC Scenarios 1-2. . . . . . . . . . . . . . . . 75

8.2 Device configurations for AC Scenarios 3-4 . . . . . . . . . . . . . . . 76

8.3 Load power settings for DC scenario 1-4 . . . . . . . . . . . . . 76

8.4 Load power settings for DC scenario 5-6 . . . . . . . . . . . . . . . 77

C.1 Measured and modeled values for system input power and transformer secondary power 105

C.2 Measured and modeled values for transformer and converter losses . . . . . . . . . . 106

C.3 Measured and modeled values for total system losses and efficiency . . . . . . . . . 107 


\section{LIST OF FIGURES}

2.1 Typical electrical distribution in a small office building $\ldots \ldots \ldots \ldots$

2.2 Current waveform from an AC/DC converter . . . . . . . . . . . . . . 8

2.3 System efficiency comparison $\ldots \ldots \ldots \ldots \ldots \ldots$

2.4 Current harmonics magnitude and phase angle . . . . . . . . . . . . . . 13

3.1 General two-port network . . . . . . . . . . . . . . . . . . 23

3.2 General one-port network . . . . . . . . . . . . . . . . . . . . . . . . . . . 24

3.3 Example electrical one- or two-port network . . . . . . . . . . . . . . . 25

3.4 Directed graph representation $\ldots \ldots \ldots \ldots \ldots \ldots \ldots \ldots$

4.1 Ideal single-phase transformer model . . . . . . . . . . . . . . . . . . . . . . 33

4.2 Equivalent circuit for single-phase transformer . . . . . . . . . . . . . . . . . . 34

4.3 Three-phase transformer component . . . . . . . . . . . . . . . . 35

4.4 Balanced electrical network with two-port linear transformer models . . . . . . . . 36

4.5 Surface plot of harmonic current magnitude . . . . . . . . . . . . . 38

4.6 Surface plot of harmonic current phase angle . . . . . . . . . . . . . . 38

4.7 Converter loss model . . . . . . . . . . . . . . . . . . . . . . . . 41

5.1 Example nonlinear network . . . . . . . . . . . . . . . . . . . . . . . 43

5.2 Annotated example nonlinear network . . . . . . . . . . . . . . . . . . . 44

5.3 Simulation results for example network (complex impedance) . . . . . . . . . . . 48

6.1 Expanded view of Modelica toolkit library . . . . . . . . . . . . . . . . . . . . 52

$6.2 \mathrm{AC} / \mathrm{DC}$ converter graphics layer $\ldots \ldots \ldots \ldots \ldots \ldots \ldots \ldots$

6.3 Impedance element . . . . . . . . . . . . . . . . . . . . . . 56

6.4 Three-phase transfomer model $\ldots \ldots \ldots \ldots \ldots \ldots$

6.5 Example system in Modelica . . . . . . . . . . . . . . . . . . . . . . . . 60

7.1 Notional rectifier circuit. . . . . . . . . . . . . . . . . . . . . . . 62

7.2 Rectifier input current vs. power level. . . . . . . . . . . . . . . . . . . . 64

7.3 Device specific behavioral model fits for magnitude and angle $\ldots \ldots \ldots \ldots$

7.4 Validation result for device behavior model characterization . . . . . . . . . . . . 71

8.1 Notional diagram of testbed, AC configuration . . . . . . . . . . . . . 73

8.2 Notional diagram of testbed, DC configuration . . . . . . . . . . . . . . 73

8.3 Testbed setup image . . . . . . . . . . . . . . . . . . . . . . . . 74

8.4 BEEAM model (AC distribution) . . . . . . . . . . . . . . . . 78

8.5 BEEAM model (DC distribution) . . . . . . . . . . . . . . . . . . . . . . 79

8.6 Predicted versus measured total system efficiencies, Scenarios 1-2 . . . . . . . . . 79

8.7 Predicted versus measured total system efficiencies, Scenarios 3-4 f . . . . . . . . . 80

8.8 Predicted versus measured total system efficiencies, Scenarios $5-6 \ldots \ldots$. . . . . . 80

8.9 Predicted versus measured total system power losses, Scenarios 1-2 . . . . . . . . . 81

8.10 Predicted versus measured total system power losses, Scenarios 3-4 . . . . . . . . . 81 
8.11 Predicted versus measured total system power losses, Scenarios 5-6 . . . . . . . 82

A.1 Phase shift in nonlinear load voltage and current phasor angle . . . . . . . . . . 95

C.1 Predicted vs measured total system input power, Scenario 1-2 . . . . . . . . . . . 108

C.2 Predicted vs measured total system input power, Scenario 3-4 . . . . . . . . . . . . . 108

C.3 Predicted vs measured total system input power, Scenario 5-6 . . . . . . . . . . . . . 109

C.4 Predicted vs measured transformer power at secondary, Scenario 1-2 . . . . . . . . . 109

C.5 Predicted vs measured transformer power at secondary, Scenario 3-4 . . . . . . . . . . 109

C.6 Predicted vs measured transformer power at secondary, Scenario 5-6 . . . . . . . . . 110

C.7 Predicted versus measured transformer loss, Scenario 1-2 . . . . . . . . . . . . . 110

C.8 Predicted versus measured transformer loss, Scenario 3-4 . . . . . . . . . . . . . . 110

C.9 Predicted versus measured transformer loss, Scenario 5-6 . . . . . . . . . . . . . 111

C.10 Predicted vs measured converter loss, Scenario 1-2 . . . . . . . . . . . . . . 111

C.11 Predicted vs measured converter loss, Scenario 3-4 . . . . . . . . . . . . . . . 111

C.12 Predicted vs measured converter loss, Scenario $5-6 \ldots \ldots$. . . . . . . . . . 112 


\section{Chapter 1}

\section{Introduction}

Until the advent and widespread use of semiconductor based power electronic devices, electrical loads consisted of resistive loads, such as, heater coils, incandescent lights; and inductive loads, such as, induction motors etc. However in the past few decades, there has been a considerable shift in the load types. Semiconductor based power electronic devices have seen an ever increasing usage. These semiconductor based power electronic devices primarily power a DC load. These newer residential electric loads, miscellaneous electric loads (MELs), and building equipment, that are based on power electronics, can operate directly from a power source DC. Power electronic devices that operate on an $\mathrm{AC}$ distribution requires an $\mathrm{AC}$ to $\mathrm{DC}$ conversion. This conversion results in power loss and thus has an overall impact of decrease in system efficiency. If a majority of loads in a distribution are DC, a logical step towards a greater system efficiency would be to eliminate the conversion step. This has resulted in considerable interest in comparing the efficiency of DC distribution systems versus traditional AC distribution. In addition, many renewable power generation systems such as solar photovoltaic (PV) and energy storage systems natively produce DC power, making conversions between AC and DC potentially unnecessary and a possible source of energy savings [1-10]. This has resulted in an increased interest of government agencies in supporting funding for research in the field of DC distribution.

This transition away from an AC only distribution has many challenges. AC has advantages that cannot be overcome by a DC only distribution. A mixed AC/DC distribution can be a transitional step towards a DC dominated distribution system. The benefits of a transition to a DC distribution can be in terms of increased efficiency or overall reduced lifecycle cost. However, it becomes a challenge to accurately quantify the benefits of these distributions because of the number of possible electrical configurations, how the system boundaries are defined, whether the application

includes an on-site storage and/or combination of potential loads and equipment types [11,12]. By leveraging the power and flexibility of advanced simulation techniques, and using accurate 
representative models for devices, one can simulate and model these configurations. This would allow building designers and electrical engineers to analyze, and compare the performance of electrical distribution systems.

This dissertation describes a modeling framework for AC, DC electrical and hybrid AC/DC distribution networks, that is packaged in a software tool called Building Electrical Efficiency Analysis Model (BEEAM) [13]. The modeling framework is developed in Modelica, an objectoriented, multi-domain, systems modeling language. Benefits of the modeling framework are: a simulation and modeling framework for harmonic analysis, and harmonic power flow studies in AC distributions containing nonlinear devices; a simulation platform for developing advanced harmonic models for nonlinear devices; ability to leverage general purpose simulation and computation tools from the Modelica language for the purpose of implementing advanced simulations. Simulation models created using modeling toolkit are validated against laboratory experiments and is shown to estimate system power and losses within reasonable degree of accuracy.

\subsection{Research Contribution}

This dissertation fills an important role in the realm of electrical power systems modeling tools. The modeling toolkit combines the advantages of object-oriented modeling with the freedom of a standardized language. The modeling toolkit presented here has the potential of acting as a test bench for future research into harmonic analysis and harmonic model development.

Major contributions of this dissertation are:

- Description of a framework and modeling approach for assessment of component and system electrical efficiencies in AC, DC and hybrid AC/DC distribution configurations, including nonlinear effects and potential load imbalances, which does not rely on time-domain simulation.

- Proposal of a new software tool (developed in Modelica, an object-oriented, multi-domain, systems modeling language) [13] that implements the above approach to perform detailed AC vs. DC distribution efficiency comparison. 
- Initial validation of the tool's accuracy with experimental measurements, for several circuit configurations and power levels on both AC and DC distribution systems, including balanced and unbalanced load conditions.

- Proposal of an analytical model for nonlinear device behavior and it's characterization procedure.

\subsection{Organization}

The remainder of this dissertation is organized as follows: The second chapter of this dissertation lays down the motivation for the research presented in this dissertation and background on relevant research on the topic of DC distribution efficiency analysis. The chapter goes into detail on background of harmonic power flow analysis technique. The third chapter describes the modeling framework used to model electrical networks and the general harmonic power flow solution for an AC distribution.

The fourth chapter is on the device models constructed using the modeling framework. It includes models for commonly encountered devices in an AC/DC electrical distribution. The chapter describes the underlying mathematical equations for the devices. It is followed by a chapter on an example electrical system that demonstrates the use of modeling framework. It describes the implementation of the network equations using the modeling framework and system solution implementation in a mathematical scripting language.

The sixth chapter describes the modeling framework implementation in an object-oriented, multi-domain, systems modeling language called Modelica. The next chapter describes a proposed model for device specific behavior models for nonlinear AC/DC converter device and lays down a characterization procedure using optimization techniques, followed by model verification studies.

The modeling toolkit is validated against elaborate laboratory experiments. Chapter eight describes the experimental setup and the experimental validation of the toolkit using laboratory measurements. Efficacy of the toolkit in predicting losses in electrical distributions is shown using plots for various experimentation scenarios. 
The end of the dissertation contains appendices for supplemental material in chapters 5 and 6 . Appendix A describes the equations for the nonlinear load used in the electrical distribution system example. Appendix B provides the source code for the example distribution solution implemented in MATLAB and equivalent Modelica code for simulation model built using the toolkit. Appendix $\mathrm{C}$ lists tables and figures from the various experimental measurements and simulation runs.

\subsection{Nomenclature and Notation}

\subsubsection{Notation}

Following notation is used throughout the document. Bold faced letters are used to denote matrices or vectors; non-bold letters are used for scalar quantities; subscripts are used to index device, port or harmonic numbers; $\mathbb{W}$ denotes the set of whole numbers; $\mathbb{C}$ denotes the set of complex numbers; $\mathcal{C}^{k}$, denotes the space of continuous functions with $k$ derivatives; $\{\cdot\}^{T}$ represents the matrix transport operator; $|\cdot|$ symbolizes the absolute value of a real number or cardinality of a set; for any set $\mathcal{S}, \mathcal{S}^{C}$ denotes its complement; $\widehat{\{\cdot\}}$ symbolizes estimated value; $\overline{\{\cdot\}}$ denotes average value or set negation, depending on context; $\widetilde{\{\cdot\}}$ denotes a phasor quantity, $\mathbb{R e}\{\cdot\}$ and $\operatorname{Im}\{\cdot\}$ symbolize real and imaginary parts of a complex number, respectively; $\|\cdot\|$ represents the magnitude of a complex number (given complex number, $x=a+j b,\|x\|=\sqrt{a^{2}+b^{2}}$ ); $\angle \cdot$ represents the argument (angle) of a complex number $\left(\angle x=\tan ^{-1}(b / a)\right) ;\{\}^{*}$ denotes complex conjugation; $j:=\sqrt{-1} ; f(z ; \alpha)$ symbolizes the evaluation of function $f$ with input variable(s) $x$ and fixed parameter(s) $\alpha$.

\subsubsection{Nomenclature}


Table 1.1: Indices and Sets.

\begin{tabular}{ll}
\hline Symbol & Description \\
\hline Indices and Sets & \\
$h \in \mathcal{H}$ & Set of harmonic numbers \\
$v \in \mathcal{V}$ & Set of one-port in electrical model \\
$w \in \mathcal{W}$ & Set of two-port networks in electrical model \\
$d \in \mathcal{D}$ & Set of all devices in the distribution model, $d \in \mathcal{V} \cup \mathcal{W}$ \\
$g \in \mathcal{G}$ & Set of subgraphs formed by interconnects between network ports \\
$p \in\{1,2\}$ & Set of device port numbers \\
$E_{g}$ & Number of edges (branches) in a subgraph $g$ \\
$N_{g}$ & Number of nodes in a subgraph $g$ \\
$E$ & Total number of edges (branches) across all subgraphs, $E=\sum_{g \in \mathcal{G}} E_{g}$ \\
$N$ & Total number of nodes across all subgraphs, $N=\sum_{g \in \mathcal{G}} N_{g}$ \\
\hline
\end{tabular}

Table 1.2: Table of variables.

\begin{tabular}{ll}
\hline Symbol & Description \\
\hline Variables & \\
$\widetilde{I}_{d, p, h}$ & Current into port $p$ of device $d$ at harmonic $h$ \\
$\widetilde{V}_{d, p, h}$ & Voltage at port $p$ of device $d$ at harmonic $h$ \\
$P_{\mathrm{in}, d}$ & Average real input power, into device $d$, at port $p$ \\
$P_{\text {out }, d}$ & Average real output power, out of device $d$, at port $p$ \\
$P_{\mathrm{in}, d, p, h}$ & Average real input power, into device $d$, at port $p$ and harmonic h \\
$P_{\text {loss }, d}$ & Average power loss in device $d$ \\
$H$ & Total harmonics in the system \\
\hline
\end{tabular}

Table 1.3: List of functions and parameters.

\begin{tabular}{ll}
\hline Symbol & Description \\
\hline Parameters & \\
$f_{d}$ & Loss function for device $d$ \\
$g_{d}$ & Device specific behavior for device $d$ \\
$\gamma_{d}$ & Vector of behavioral parameters for device $d$ \\
$\boldsymbol{\beta}_{d}$ & Vector of one-state loss parameters for device $d$ \\
$\gamma_{d}$ & Vector of two-state loss parameters for device $d$ \\
\hline
\end{tabular}




\section{Chapter 2}

\section{Background and Related Work}

This chapter describes electrical distribution components and terminology associated with energy efficiency calculations, followed by literature review on harmonic power flow. To quantify losses in an electrical distribution, one must first describe an electrical distribution and constituent devices.

\subsection{Losses in an Electrical Distribution}

Consider a typical electrical distribution in a building, as shown in Figure 2.1. The building electrical distribution is connected to the utility grid, shown on the left. The grid is assumed to be stiff with no voltage distortion and hence no voltage harmonics. Buildings connect to the utility grid through a step down transformer. Electrical distribution for small electrical appliances run at $208 \mathrm{Y} / 120 \mathrm{~V}$, whereas 480Y/277 V distribution is used for higher power systems such as HVAC loads, etc.

Electrical loads in a building distribution can be grouped into the following categories:

- Linear electric loads such as space heaters, water heaters. These are linear devices and are modeled as resistive loads.

- Inductive loads such as motors (for ventilation fans, pumps, etc.) These are electromechanical devices that convert electricity into useful mechanical power.

- Lighting loads (Light Emitting Diode) powered by a power electronic converters. This includes florescent lights (newer devices are powered by electronic ballast).

- Miscellaneous Electronic Loads (MELs) powered by power an AC/DC electronic converter. These are DC devices such as computers, phone chargers, printers, computer monitors, etc. 


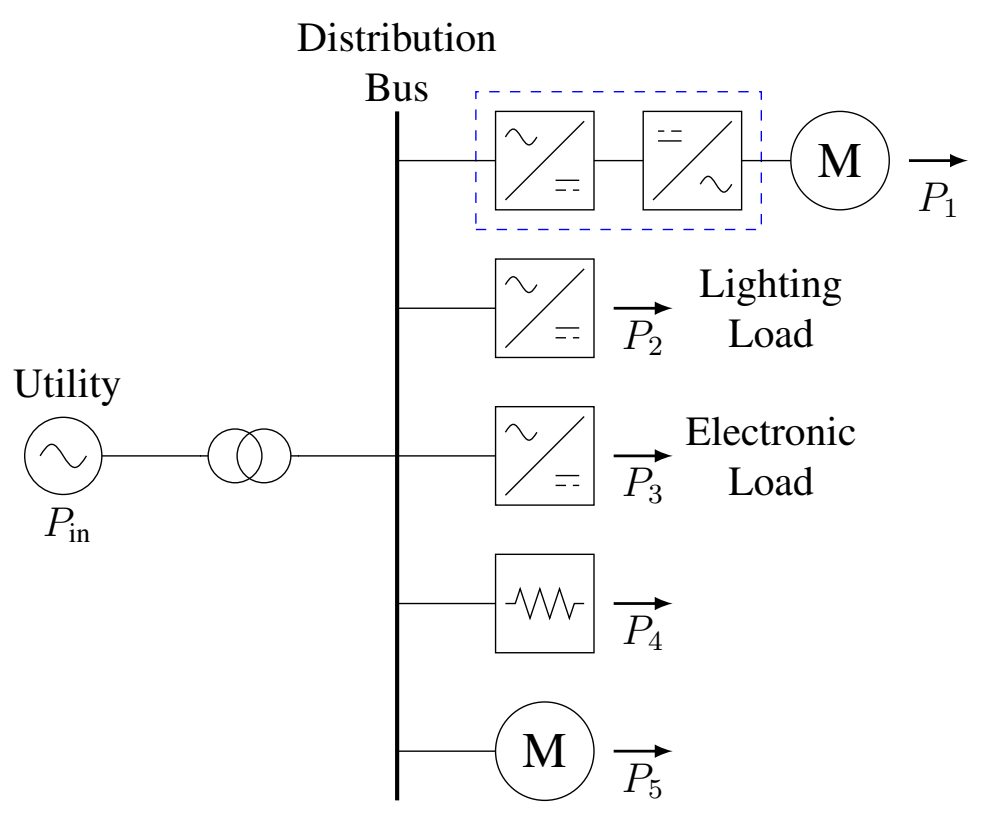

Figure 2.1: Typical electrical distribution in a small office building.

- Inductive loads powered by a variable frequency drive (VFD). VFD (shown as blue box) consist of an AC/DC converter followed by an inverter stage (DC/AC converter). Example of such devices would be, some newer home appliance motors, HVAC systems, etc.

Losses can be grouped into four main categories, namely, transformer losses, losses resulting from distribution wires, power converter losses and losses in electrical machines. Figure 2.1 shows the output power from all the end point user loads, shown as $P_{i}, i \in\{1, \ldots, 5\}$. Total power into the distribution at the point of utility connection is shown as $P_{\text {in }}$. System efficiency is defined as the ratio of total power consumed by loads to the total input power.

The proliferation of DC loads has resulted in considerable shift in the overall electrical loads with the MELs and lighting loads constituting majority of the loads. This has resulted in considerable interest in DC distributions [11] [3]. Variations in electrical distributions have been proposed that replace the multiple conversion stages and thus predict an improved system efficiency. 


\subsection{Effect of Nonlinear Loads on Energy Efficiency}

DC loads in an AC distribution are powered by an AC/DC converter. The simplest of these converters (rectifiers) convert the $\mathrm{AC}$ voltage to $\mathrm{DC}$ using a bridge rectifier constructed using diodes. The rectification process is nonlinear, and results in current waveform distortion [14] [15]. Therefore, AC/DC converters are nonlinear loads that cannot be modeled using conventional fundamental power flow analysis techniques. Figure 2.2 shows the current waveform of a typical AC/DC converter. The peaks in the figure correspond to the conduction period for the rectifier diodes. Additional passive elements in the converter, such as line filters, common mode chokes, etc., might result in further waveform distortion. This distortion in the current waveform results in an increase in the fundamental current harmonic component (increase in the system rms current) that in turn results in higher losses in an electrical distribution.

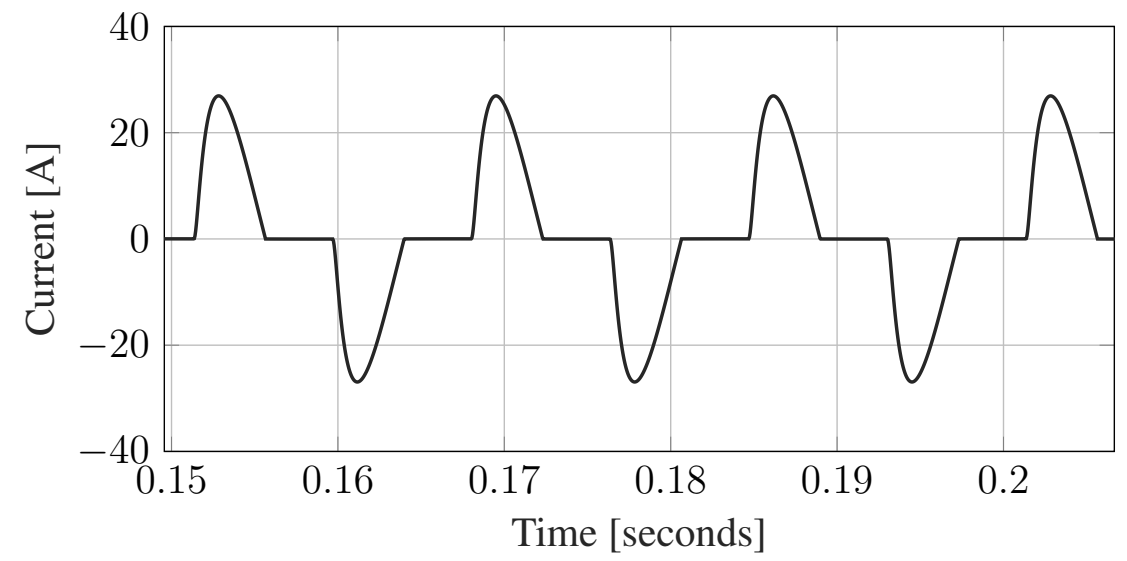

Figure 2.2: Current waveform from an AC/DC converter.

The nonlinearity associated with an AC/DC converter results in under estimation of the system losses. The effect of nonlinear devices on the overall system can be best understood by a simple example.

Figure 2.3 shows the setup of a simulation containing two loads connected via an impedance to a stiff voltage source such as a utility power grid. This impedance may represent any line impedance in a distribution, such as cable impedance, transformer winding etc. The sinusoidal 
source here is assumed to be ideal with no source impedance. The goal is to compare efficiencies of the two systems, therefore, the output power is measured at the point of use. For the nonlinear load, the output power is measured at the DC side of the rectifier (assuming zero converter losses). For the linear AC load, the output power is measured at the input.
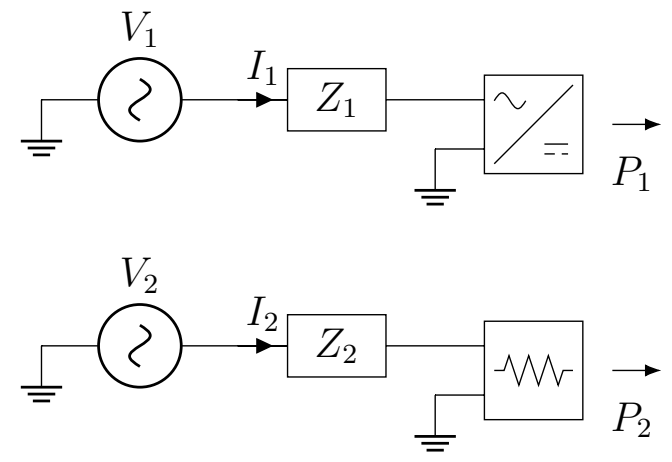

Figure 2.3: System efficiency comparison for a linear and nonlinear load.

Both of the end point devices are controlled loads where the output power can be as a model parameter. The simulation parameters are as follows, $Z_{1}=Z_{2}=1.5 \Omega, V_{1}=V_{2}=120 \mathrm{~V}$ (rms), $P_{1}=P_{2}=1000 \mathrm{~W}$. The time domain simulation was run till the system reaches steady state and the system efficiency was computed. The resulting system efficiencies for the two systems is summarized in the Table 2.1 .

Table 2.1: Comparison of system efficiencies for linear and nonlinear load.

\begin{tabular}{lcc}
\hline & Nonlinear load & Linear Load \\
\hline Output power (W) & 1000 & 1000 \\
System input power (W) & 1285.10 & 1135.07 \\
System Efficiency & $77.81 \%$ & $88.09 \%$ \\
\hline
\end{tabular}

Assuming the converter is lossless, for the same output power, the system with the nonlinear results in lower system efficiency (higher system losses) than the linear load. Modeling of electrical 
distributions using convention techniques, that contain large number of nonlinear loads would therefore underestimate system losses.

Results from the above simulation suggest that a system containing nonlinear loads cannot be accurately modeled in a conventional power flow analysis as one is ignoring the effect of current distortion resulting from the nonlinear load. Therefore, there is a need for a power flow formulation that can model the nonlinearities but at the same time provide simulation advantages of a frequency domain based power flow simulation. Harmonic power flow is an extension of the power flow formulation for AC electrical distributions containing harmonic producing loads.

\subsection{Energy Efficiency Modeling Techniques}

Energy efficiency modeling in electrical distribution systems can be achieved using different approaches. Relevant modeling approaches are, fundamental power flow, harmonic power flow (HPF), energy balance approach based on device efficiency curves and full time-domain simulation. Here is a brief overview of these modeling techniques

Energy balance modeling technique is the simplest in which the loads and component losses are tallied to estimate the required input energy. A simple implementation would use basic system topologies and fixed device efficiencies to estimate the potential energy savings of DC distributions verses AC [8,16-18]. Energy balance models are insufficient as they do not directly model voltage, current and power flows within the distribution network. Therefore, they do not generally provide other measures of system performance (voltage drop, power quality, and the like). It can also yield inaccurate estimates of efficiency to the extent that device losses are influenced by phenomena such as voltage, system imbalance, and current distortion.

Fundamental power flow is another technique for modeling electrical distributions. Knowing the amount of power drawn by the end loads, one can solve the system to get voltage drops across the network, power consumed at the source, etc. [19]. Though accurate for utility level electrical distribution modeling, is insufficient for systems containing a large number of nonlinear devices, such as building electrical distribution systems containing power electronic loads [20]. As demon- 
strated in the previous section, a fundamental power flow analysis (with the assumption of linear device models in the distribution system) would underestimate power consumption from nonlinear loads.

Time-domain simulations are the most accurate as they rely on time domain models of electrical loads. Time domain simulation can model any type of nonlinearity in the system. For an accurate estimation of system performance, a time domain simulation must be allowed to settle down since the initial conditions of the numerical solver can result in different system behavior. The simulation output is allowed to settle down until the system is in steady state operation and there are no system wide transients. The downside of time domain simulation is the computational time and resource penalty. This makes time domain simulation unsuitable for large simulation models. The remaining three modeling approaches were compared and contrasted in [21] for estimating efficiencies in AC-only, DC-only and hybrid AC/DC distribution systems. Therein, comparison between modeling results and experimental measurements supported the conclusion that the HPF method provided a suitable balance between accuracy, computation time, and model development compared to the other approaches. In addition, the HPF method provides the advantages of predicting harmonic content, simulation of highly unbalanced load conditions in three phase systems, and the ability to simulate potentially large networks.

The next section gives a brief overview of frequency domain representation of a system containing non-sinusoidal signals and the concept of harmonics.

\subsection{Frequency-domain Representation of Non-sinusoidal Sig-}

\section{nals}

Consider a non-sinusoidal signal $x(t)$ having a period $T_{0}$ (frequency $\omega_{0}$ ). Such a signal can be written in the form of a Fourier series expansion as [22] [23]

$$
x(t)=\sum_{n=0}^{\infty} c_{n} e^{j n \omega_{0} t}
$$


where

$$
c_{n}=\frac{1}{T_{0}} \int_{0}^{T_{0}} x(t) e^{\left(-j n \omega_{0} t\right)} d t
$$

The Fourier coefficients $c_{n} e^{j n \omega_{0} t}$ are phasors with frequency that is the integer multiple of the frequency of the periodic signal (fundamental frequency).

In practice, signal processing is carried out in computers that are digital devices. To capture a continuous signal, the signal is first measured using a transducer and captured using an analog to digital converter (ADC). The signal is captured using an ADC at a fixed sampling rate. Let the sampling rate be $T_{s}$. Let $x(n)$ be a discrete signal with period $N, n=0,1, \ldots, N-1$. The discrete Fourier transform (DFT) of the signal $x(n)$ is define as:

$$
X(k)=\sum_{n=0}^{N-1} x(n) e^{-j 2 \pi n k / N}
$$

where, $k=0,1, \ldots, N-1 . X(k)$ are the Fourier coefficients at index $k . X(k) \in \mathbb{C}$, is a complex number with an associated magnitude and phase angle.

Therefore, a system containing nonsinusoidal signals, one can transform the signal using DFT and represent the system in the equivalent frequency domain using these 'DC' quantities (also called phasor(s), chapter 3).

A harmonic is defined as a periodic signal with frequency that is an integer multiple of the frequency of the original signal. In an electrical distribution, the frequency of the harmonics are an integer multiple of the line frequency $(50 \mathrm{~Hz}$ or $60 \mathrm{~Hz})$.

Figure 2.4 shows the spectral decomposition (harmonics components) of the current waveform from Figure 2.2. The plot at the top shows the magnitude of the harmonic components and the bottom plot shows the angle of the components vs the harmonic number. In a symmetric waveform, even harmonics are zero and can therefore be neglected. Thereafter, the simulation models would only consider odd harmonics. 

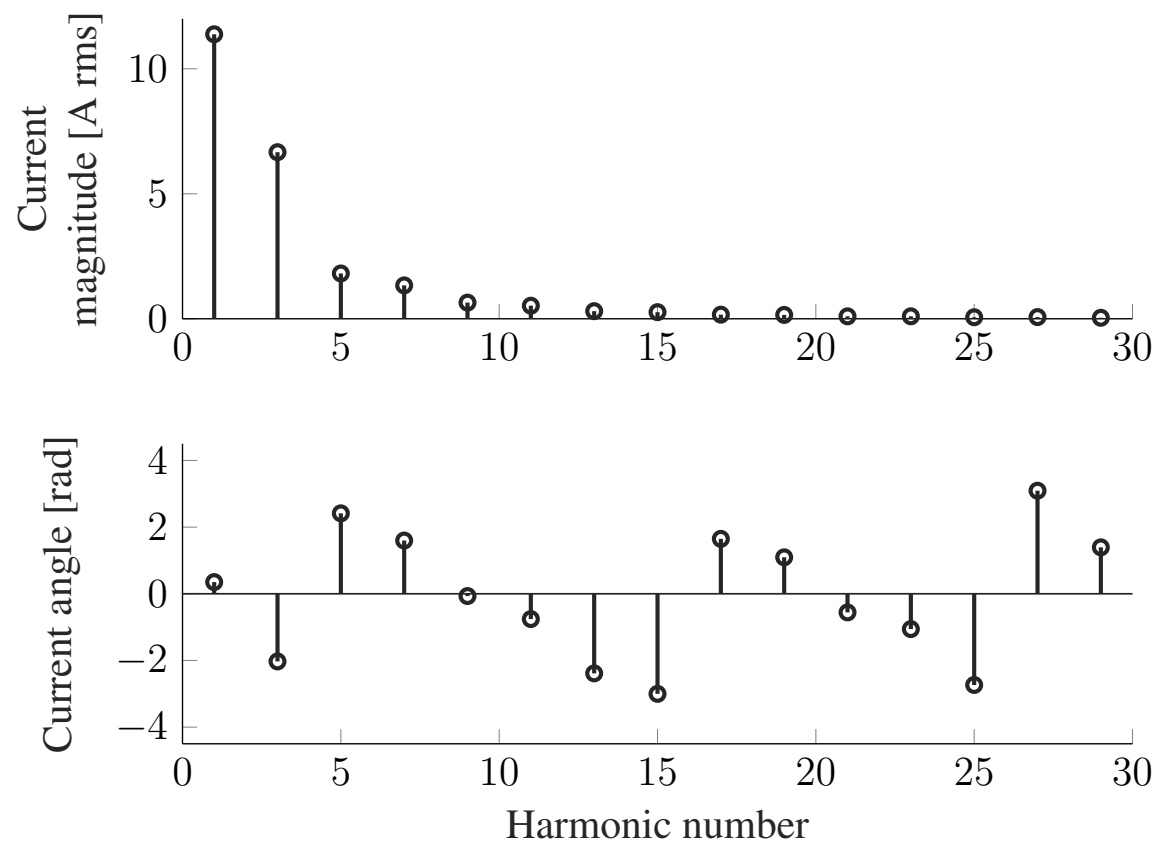

Figure 2.4: Stem plot showing current magnitude (top) and angle for odd harmonics.

\subsection{Background and Literature Review}

Nonlinear devices in an electrical distribution distort an otherwise sinusoidal voltage and current waveform. This distortion results in non-sinusoidal waveforms that propagate throughout the network. The presence of these distorted waveforms means that the system cannot be modeled and analyzed using conventional fundamental power flow techniques. Such systems can be accurately modeled in the time domain using a numerical differential equation solver. The downside of a time domain simulation is the large amount of computation time and resources that are needed. Scaling such a system to contain a large number of loads results in a simulation setup that takes up significant computation time. Therefore, there is a need of an analysis framework that can handle nonlinear devices but at the same time provide the computational speed of phasor based fundamental power flow.

\subsubsection{Harmonic Power Flow (original formulation)}

Conventional power flow is a well established and thoroughly understood technique for power system analysis. It is used to solve for system wide voltages, currents and power flows in an 
electrical distribution network. In a power flow formulation, loads are the end point devices that consume power and therefore are modeled as power sinks [19]. It is a fairly well established and researched technique with a plethora of tools and models are available for a wide variety of devices and loads. Unfortunately the technique is only useful for linear devices that are modeled at fundamental frequency and cannot be used for accurate analysis and determination of system efficiencies in distribution systems containing large number of nonlinear devices such as single phase rectifier loads [20].

Harmonic power flow is an extension of the fundamental power flow for electrical systems containing nonlinear harmonic producing devices. In fundamental power flow, the system is modeled in frequency domain at steady state using phasor representation at the fundamental frequency. Similarly, a network containing nonlinear loads can be modeled in the frequency domain by extending the system representation from a single frequency to multiple frequencies that are an integer multiple of the fundamental frequency called harmonics. Nonlinear devices are modeled in the frequency domain as harmonic producing sources. The technique was first introduced by Xia and Heydt $[24,25]$ for a balanced three phase system. The original formulation was an extension of the fundamental power flow technique, solved using the Newton-Raphson method. The technique can be summarized as, 1) Formation of a harmonic admittance matrix based on the network interconnects. A harmonic admittance matrix is similar to an admittance matrix in fundamental power flow with the difference that additional admittance terms are added corresponding to higher harmonics. 2) Form the mismatch vector and the system Jacobian. 3) Solving the simultaneous equations using the Newton update. The solver iterates until the mismatch vector is less than the tolerance in which case the solver terminates and we get the solution.

Some of the limitations of this formulation were, the algorithm was limited to balanced networks with no zero sequence signals and therefore could not handle multiphase, unbalanced systems. Another limitation was the lack of diversity in harmonic load models as the original formulation had only a single harmonic load model. Over the years, several improvements were made to the HPF formulation. The algorithm was extended to multiphase and unbalanced systems [26,27]. 


\section{HPF techniques and variations}

A system modeled using the harmonic power flow is considerably larger than a conventional fundamental power flow. An increase in the system size can result in increased computation time, increased memory requirements and issues with convergence. Convergence issues can be amplified if the system contains a large number of nonlinear loads. To circumvent these issues, several modifications have been proposed. These modified algorithms exploit the underlying structure of the system formulation to simplify solver implementation.

Harmonic power flow formulation can be either coupled or decoupled. In a system where interharmonic interactions, there is a coupling between voltages and/or currents are different harmonics. Such a system results in a system of equations that contain all the harmonics and therefore the resulting system can be quite large. In a system with negligible inter-harmonic interaction, one can solve the system, one harmonic at a time. This is called a decoupled harmonic power flow [28]. The resulting system of equations are much smaller resulting in convergence guarantee and faster solution times.

Modular harmonic power flow technique [29] relies on the fact that any inter-harmonic interaction only occurs for the nonlinear loads. Therefore, one can add modularity in the system formulation by detaching the nonlinear device models from the main program body. The nonlinear models are solved separately and interfaced with the system as harmonic sources.

These modified algorithms result in simpler systems and therefore are easier to implement and can ensure convergence. A major motivation of these algorithms has been lack of computation resources in early days of computing. With the advancement and ever-increasing computation power, these techniques do not present a significant advantage over a fully coupled harmonic power flow formulation that does not use these techniques. It then becomes an engineering judgment as to which algorithm suits the user needs. A thorough and comprehensive review on various harmonic power flow techniques can be found in [30].

A recent addition to the power system harmonic analysis is the technique using equivalent split-circuit models [31]. Network electrical devices are replaced with equivalent circuit elements. 
The system is solved using harmonic balance technique. This approach is radically different to the harmonic power flow techniques mentioned above as it does not involve an admittance matrix.

\subsubsection{Harmonic Power Flow Analysis Tools}

Currently there are only few tools available for harmonics power flow analysis. The original harmonics power flow implementation [25] resulted in a tool called HARMFLO [32] developed for the EPRI [33]. The program was written in FORTRAN and it implements a Newton-Raphson based solver. The codebase is old and the tool has not been in active development, with no accessible version of the codebase available.

ETAP is a commercial tool for power flow analysis [34]. The tool has a harmonic analysis module. Being a commercial product, the licensing would make it unsuitable for incorporating into other open source tools. ETAP does not support functional mock-up interface (FMI) [35] and therefore simulation models cannot be integrated with other simulation platforms and for performing co-simulations.

OpenDSS is an open source electrical distribution analysis software developed by EPRI [36] [37]. It is intended for utility scale fundamental power flow analysis. The tool provides an option to run harmonic analysis. User can define harmonic spectra and the system is solved. The tool lacks sophisticated harmonic device models and cannot perform a full harmonic power flow analysis.

\subsubsection{Load Models}

At the core of a harmonic power flow analysis is an accurate model of the nonlinear harmonics producing load. Though it is relatively easy to model nonlinear loads in time domain, their frequency domain counterpart are difficult to model [38]. Harmonic distortion can result from sources such as an DC/AC converter, but for this dissertation is limited to electrical distributions connected to a stiff grid that can be modeled as a pure sinusoid. In distributions that do not have source harmonics can have harmonic distortion as a result of nonlinear loads. This section covers some of the harmonic load models developed over the years. 
Harmonic power flow analysis technique was developed to analyze harmonics in electrical distribution networks resulting from three phase converters. The original harmonic power flow formulation [24] was built around a harmonic model of a six-pulse line commutated thyristor controlled converter [33]. The nonlinear load was modeled using injected current balance equations. The currents produced by the harmonic load is modeled as injection currents at bus $m$ and harmonic $h$ are given by the current balance equation, $I_{m}^{(h)}=g^{(h)}\left(v_{m}^{(1)}, v_{m}^{(5)}, \ldots \alpha_{m}, \beta_{m}\right)$, where $\alpha_{m}$ is the triggering angle at bus $m$, and $\beta_{m}$ is the commutating resistance or DC voltage. Another harmonic model for a three phase converter model can be found in [39].

Single phase full bridge rectifiers produce a signature harmonic spectrum at a fixed power and line impedance. In scenarios where the loads are not sensitive to voltage variations, one can model the nonlinear load as a bunch of constant current sources at each harmonic. The method of using constant current sources based on a reference current injection spectra is used in [3] [11] for harmonic power flow analysis.

Admittance matrix techniques utilize the fact that currents at different harmonics can be modeled by linear admittance relationships with line harmonic voltages by an admittance matrix. Nonlinear loads are modeled using a crossed frequency admittance matrix [40]. This model takes into account interaction between voltages and currents at different harmonics. This results in an admittance matrix with nondiagonal elements for a nonlinear load and a square diagonal matrix for a linear load. A similar technique uses a harmonically coupled linear admittance matrix to model thyristor controlled single and three phase bridge rectifiers [41]. This technique uses a harmonically coupled admittance matrix that models the AC side harmonics generated by the nonlinear rectification process. Electrical distributions for buildings exclusively use uncontrolled line commutated rectifiers that uses diodes and therefore the model is beyond the scope of this research.

An improvement over the fixed current injection method is the Norton equivalent model [42]. The harmonic model takes into account the effect of voltage variation on the current injection. Harmonic producing load is modeled as a Norton equivalent circuit consists of a harmonic current source in parallel to a harmonic Norton impedance. The load model is generated using mea- 
surements of the load voltage and current at different operating points. An improvement in the modeling approach involved the use of harmonically coupled impedance matrix [43].

Of the three harmonic load modeling techniques, 1) the constant current source model, 2) Norton equivalent model 3) Cross frequency admittance matrix model, Norton model has been shown to be the most accurate [44]. These harmonic load models have several shortcomings. Firstly, most of the research has been on focused of harmonics in utility level electrical distribution systems. Therefore, models of harmonic loads have been limited to force commutated converters. Secondly, these harmonic load models are usually modeled at a fixed operating point. Therefore, the resulting model fails to capture harmonic spectra for a load that operates varying power levels. Most AC/DC converters, such as, light fixtures are fixed power loads, but converters operating at varying power are becoming widespread. Such converters include computer power supplies, dimmable light fixtures, HVACs etc. This research will explore harmonic models of single phase $\mathrm{AC} / \mathrm{DC}$ converters that operate at different power levels.

Another technique of modeling a single phase rectifier is a hybrid approach, in which the rectifier current and voltage waveforms are solved in the time domain and then transformed to the frequency domain [45]. This technique relies on the single phase rectifier topology that consists of a full-bridge rectifier followed by a large filter capacitor. Given the voltage is sinusoidal, one can analytically solve for the time domain waveform. The resulting waveform can then be analyzed in frequency domain by computing Fourier series coefficients. The technique is used in [46] to analyze diversity effects and harmonics in electrical distributions containing large number of nonlinear harmonic producing loads. 


\section{Chapter 3}

\section{Modeling Framework}

This section describes the modeling framework used to estimate harmonic currents, voltages, and power flows in buildings within the BEEAM modeling toolkit. A background on electrical network theory using one- and two-port models is first given, followed by a description of the model representation of several devices.

\subsection{Background on Linear AC Analysis}

Steady-state analysis of AC circuits using frequency-based methods is a well-established technique in power engineering community. In electrical systems composed of linear and passive circuit elements, driven by sinusoidal sources, phasor analysis is often employed to reduce the solution of steady-state currents and/or voltages in a network to solving of linear algebraic equations. In this method, sinusoidal voltage and currents are represented as phasors in the frequency under the transformation:

$$
f(t)=\sqrt{2} F \cos \left(\omega t+\theta_{f}\right) \longleftrightarrow \widetilde{F}=F \angle \theta_{f},
$$

where $f$ can represent voltage or current, $\widetilde{F}$ is the phasor representation of $f, F=\|\widetilde{F}\|$ is the root-mean-square (rms) value of $f, \omega$ is the angular electrical frequency, $t$ is time, $\angle \theta_{f}$ symbolizes $e^{j \theta_{f}}$, and $\theta_{f}$ is a phase shift generally measured with respect to an input source. On the right side of (3.1), the symbol for electrical frequency is suppressed, but implied under the transformation shown. Additionally, note that the representation of the phasor magnitude as the rms value of $f$ (versus peak value) is arbitrary, but us the convention used throughout this paper.

In phasor analysis, the steady-state effect (reaction) of an inductive element $L$ in an electrical circuit is represented by an inductive reactance, $X_{L}=\omega L$, and capacitive element $C$ by capacitive reactance, $X_{C}=1 / \omega C$. Resistive elements do not have a frequency dependence and are therefore 
not transformed under phasor analysis. Impedance $Z=R+j X=\|Z\| \angle \theta_{z}=\|Z\| e^{j \theta_{z}}$ is a complex number with total resistance $R$ and total reactance $X$; these quantities may consist of combined resistances, inductive reactances, and capacitive reactances, computed according to network circuit laws.

Let $\widetilde{\boldsymbol{V}}=\left[\widetilde{V}_{1} \cdots \widetilde{V}_{N}\right]^{T}$ denote a vector of phasors containing phasor voltages $\widetilde{V}_{n}$ at nodes $n=1,2, \ldots, N$ in an electrical network; $\widetilde{\boldsymbol{I}}=\left[\widetilde{I}_{1} \cdots \widetilde{I}_{M}\right]^{T}$ denote a vector of phasors containing phasor currents $\widetilde{I}_{m}$, through branches $m=1,2, \ldots, M$ in the network. Let $\boldsymbol{Z}$ denote an $N \times$ $M$ impedance matrix which specifies the network impedance relations between node voltage and branch current phasors, i.e., $\widetilde{\boldsymbol{V}}=Z \widetilde{\boldsymbol{I}}$. For a linear network, the solution of steady-state current phasors can be computed (assuming $\boldsymbol{Z}$ is invertible) as:

$$
\widetilde{\mathbf{I}}=\mathbf{Z}^{-1} \widetilde{\mathbf{V}}=\mathbf{Y} \widetilde{\mathbf{V}}
$$

where $\boldsymbol{Y}$ is called the admittance matrix. Although phasor analysis in power engineering is generally applied at one frequency (e.g., line frequency of the voltage source), under the assumption of system linearity, (3.2) can be applied at any frequency to determine an overall steady-state response. Letting $h \in \mathbb{W}$ denote the harmonic number with respect to base frequency $\omega$, phasors corresponding to voltages and currents with electrical angular frequency $h \omega$ can be transformed by generalizing (3.1) as:

$$
f(t)=\sqrt{2} F_{h} \cos \left(h \omega t+\theta_{f, h}\right) \longleftrightarrow \widetilde{F}_{h}=F_{h} \angle \theta_{f, h}
$$

where $F_{h}=\left\|\widetilde{F}_{h}\right\|$ is the root-mean-square (rms) value of $f$ at harmonic $h, h \omega$ is the implied angular electrical frequency of phasor $\widetilde{F}_{h}$, and $\theta_{f, h}$ is the phase shift at this harmonic number. Note that while $h=0$ (i.e., DC) is valid in (3.3), there is no phase shift and $\sqrt{2}$ term is omitted.

Let $\mathcal{H}=\left\{h_{0}, h_{1}, h_{2}, \ldots, h_{H}\right\}, h_{i} \in \mathbb{W}$ be a set of harmonic numbers which are to be evaluated, where herein it is assumed that $H \geq 2$. The solution of the linear network equations at each harmonic $h$ can be computed individually by generalizing (3.2) by the use of (3.3) as: 


$$
\widetilde{\mathbf{I}}_{h}=\mathbf{Y}_{h} \widetilde{\mathbf{V}}_{h}, \forall h \in \mathcal{H}
$$

where $\boldsymbol{Y}_{h}$ is the admittance matrix at harmonic $h$. While the solution given by the phasor approach in (3.4) is simple to implement, the method can unfortunately not be used in applications where there are nonlinear relationships between system voltages and currents, such as electrical systems containing typical power electronic devices. Following the discussion below on one- and tow-port network theory, the solution of these systems using harmonic power flow is discussed (chapter 4)

\subsection{Electrical Network Theory}

\subsubsection{One- and Two-Port Network Theory}

A two-port network, depicted in Figure 3.1, is an electrical network with tow "ports", each consisting of two terminals (four terminals total). The voltage/current relationships between the terminals are described using $2 \times 2$ matrices; the two voltages and two currents are state variables, two of which are considered independent (or input) and two of which are considered dependent (or output). Note that in this context, "input" and "output" are defined for computational purposes only and not necessarily indicate the direction of real power flow. Herein, port 1 (port 2) is arbitrarily assigned the input (output) port label. The "ABCD" representation is a common representation of a two-port network; this representation for the device in Figure 3.1 is given as:

$$
\left[\begin{array}{l}
\widetilde{V}_{1} \\
\widetilde{I}_{1}
\end{array}\right]=\left[\begin{array}{ll}
A & B \\
C & D
\end{array}\right]\left[\begin{array}{c}
\widetilde{V}_{2} \\
-\widetilde{I}_{2}
\end{array}\right]
$$

In linear devices, coefficients $A, B, C$, and $D$ in (3.5) are generally complex constants. Note that in this formulation, a positive (negative) sign indicates that current is flowing into (out of) the device; this convention explains the negative sign on $\widetilde{I}_{2}$ in (3.5). See [47] for other equally-valid network representations. 
To account for additional harmonics, the tow-port matrix representation can be extended to represent harmonic voltage and current relationships. This is done by expressing the voltages and currents as vectors of phasors at each harmonic frequency (including $h=0$ for DC). To do this, let $\widetilde{I}_{d, p, h}$ and $\widetilde{V}_{d, p, h}$ represent the current and voltage phasors, respectively, for device $d$ at port $p \in 1,2$ and harmonic $h . A, B, C$, and $D$ and the new matrices are size $H \times H$. For example, to relate input $\widetilde{V}_{d, 2, h^{\prime}}$ with output $\widetilde{V}_{d, 1, h^{\prime}}$, define matrix element $A_{d, h, h^{\prime}}$, in which $h^{\prime}$ is the harmonic order associated with the input phasor and $h$ is the harmonic order associated with the output phasor, and similarly for matrix elements $B_{d, h, h^{\prime}}, C_{d, h, h^{\prime}}, D_{d, h, h^{\prime}}$. The two-port input/output relationship is for all harmonics is then given as:

$$
\left[\begin{array}{c}
\widetilde{V}_{d, 1, h_{0}} \\
\widetilde{V}_{d, 1, h_{1}} \\
\vdots \\
\widetilde{V}_{d, 1, h_{H}} \\
\widetilde{I}_{d, 1, h_{0}} \\
\widetilde{I}_{d, 1, h_{1}} \\
\vdots \\
\widetilde{I}_{d, 1, h_{H}}
\end{array}\right]=\left[\begin{array}{cccc|cccc}
A_{d, h_{0}, h_{0}} & A_{d, h_{0}, h_{1}} & \cdots & A_{d, h_{0}, h_{H}} & B_{d, h_{0}, h_{0}} & B_{d, h_{0}, h_{1}} & \cdots & B_{d, h_{0}, h_{H}} \\
A_{d, h_{1}, h_{0}} & A_{d, h_{1}, h_{1}} & \cdots & A_{d, h_{1}, h_{H}} & B_{d, h_{1}, h_{0}} & B_{d, h_{1}, h_{1}} & \cdots & B_{d, h_{1}, h_{H}} \\
\vdots & \vdots & \ddots & \vdots & \vdots & \vdots & \ddots & \vdots \\
A_{d, h_{H}, h_{0}} & A_{d, h_{H}, h_{1}} & \cdots & A_{d, h_{H}, h_{H}} & B_{d, h_{H}, h_{0}} & B_{d, h_{H}, h_{1}} & \cdots & B_{d, h_{H}, h_{H}} \\
\hline C_{d, h_{0}, h_{0}} & C_{d, h_{0}, h_{1}} & \cdots & C_{d, h_{0}, h_{H}} & D_{d, h_{0}, h_{0}} & D_{d, h_{0}, h_{1}} & \cdots & D_{d, h_{0}, h_{H}} \\
C_{d, h_{1}, h_{0}} & C_{d, h_{1}, h_{1}} & \cdots & C_{d, h_{1}, h_{H}} & D_{d, h_{1}, h_{0}} & D_{d, h_{1}, h_{1}} & \cdots & D_{d, h_{1}, h_{H}} \\
\vdots & \vdots & \ddots & \vdots & \vdots & \vdots & \ddots & \vdots \\
C_{d, h_{H}, h_{0}} & C_{d, h_{H}, h_{1}} & \cdots & C_{d, h_{H}, h_{H}} & D_{d, h_{H}, h_{0}} & D_{d, h_{H}, h_{1}} & \cdots & D_{d, h_{H}, h_{H}}
\end{array}\right]\left[\begin{array}{c}
\widetilde{V}_{d, 2, h_{0}} \\
\widetilde{V}_{d, 2, h_{1}} \\
\vdots \\
\widetilde{V}_{d, 2, h_{H}} \\
-\widetilde{I}_{d, 2, h_{0}} \\
-\widetilde{I}_{d, 2, h_{1}} \\
\vdots \\
-\widetilde{I}_{d, 2, h_{H}}
\end{array}\right]
$$

Note that for purely linear devices (such as cables), there is no cross-coupling of harmonics and all off-diagonal elements of the submatrices are zero: 


$$
\left[\begin{array}{c}
\widetilde{V}_{d, 1, h_{0}} \\
\widetilde{V}_{d, 1, h_{1}} \\
\vdots \\
\widetilde{V}_{d, 1, h_{H}} \\
\widetilde{I}_{d, 1, h_{0}} \\
\widetilde{I}_{d, 1, h_{1}} \\
\vdots \\
\widetilde{I}_{d, 1, h_{H}}
\end{array}\right]=\left[\begin{array}{cccc|cccc}
A_{d, h_{0}, h_{0}} & 0 & \cdots & 0 & B_{d, h_{0}, h_{0}} & 0 & \cdots & 0 \\
0 & A_{d, h_{1}, h_{1}} & \cdots & 0 & 0 & B_{d, h_{1}, h_{1}} & \cdots & 0 \\
\vdots & \vdots & \ddots & \vdots & \vdots & \vdots & \ddots & \vdots \\
0 & 0 & \cdots & A_{d, h_{H}, h_{H}} & 0 & 0 & \cdots & B_{d, h_{H}, h_{H}} \\
\hline C_{d, h_{0}, h_{0}} & 0 & \cdots & 0 & D_{d, h_{0}, h_{0}} & 0 & \cdots & 0 \\
0 & C_{d, h_{1}, h_{1}} & \cdots & 0 & 0 & D_{d, h_{1}, h_{1}} & \cdots & 0 \\
\vdots & \vdots & \ddots & \vdots & \vdots & \vdots & \ddots & \vdots \\
0 & 0 & \cdots & C_{d, h_{H}, h_{H}} & 0 & 0 & \cdots & D_{d, h_{H}, h_{H}}
\end{array}\right]\left[\begin{array}{c}
\widetilde{V}_{d, 2, h_{0}} \\
\widetilde{V}_{d, 2, h_{1}} \\
\vdots \\
\widetilde{V}_{d, 2, h_{H}} \\
\widetilde{I}_{d, 2, h_{0}} \\
\widetilde{I}_{d, 2, h_{1}} \\
\vdots \\
\widetilde{I}_{d, 2, h_{H}}
\end{array}\right]
$$

in which case, the two-port network representation is fully decoupled by harmonic and can be written compactly as:

$$
\left[\begin{array}{c}
\widetilde{V}_{d, 1, h} \\
\widetilde{I}_{d, 1, h}
\end{array}\right]=\left[\begin{array}{ll}
A_{d, h, h^{\prime}} & B_{d, h, h^{\prime}} \\
C_{d, h, h^{\prime}} & D_{d, h, h^{\prime}}
\end{array}\right]\left[\begin{array}{c}
\widetilde{V}_{d, 2, h} \\
-\widetilde{I}_{d, 2, h}
\end{array}\right]
$$

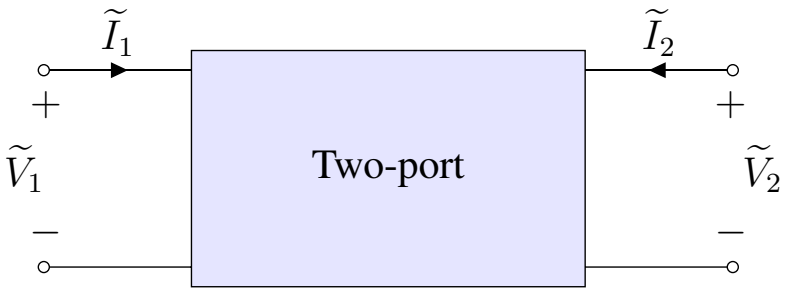

Figure 3.1: General two-port network.

Nonlinear devices, however may contain coupling between harmonics. Furthermore, the governing equations for practical nonlinear devices cannot always be described using the standard two-port network form. (The matrix elements may be functions of the state variables, or the matrix representation may not be an adequate way to capture nonlinear relationships.) To generalize the 
two-port network concept for nonlinear devices, a "general nonlinear two-port network model" for any arbitrary device $d \in \mathcal{D}$ is proposed herein which satisfies the following properties:

- The model specifies $2 H$ voltage phasor variables $\left(\widetilde{V}_{d, 1, h}\right.$ and $\left.\widetilde{V}_{d, 2, h}\right)$ and $2 \mathrm{H}$ current phasor variables $\left(\widetilde{I}_{d, 1, h}\right.$ and $\left.\widetilde{I}_{d, 2, h}\right), \forall h \in \mathcal{H}$;

- The model defines a set of exactly $2 H$ independent nonlinear equations in the variables above that describe the device behavior;

- The nonlinear equations that define the device behavior can be written to describe exactly two independent variables and two dependent variables at each harmonic $h$.

Note that with these properties, a traditional linear two-port network is a specific case of the general nonlinear two-port network model described above. The network formulation used in this research also utilizes one-port networks for sources, and loads, Figure 3.2, which have a single voltage, and single current and a (linear or nonlinear) relationship between them. A one-port network consists of $H$ phasor voltage variables, $H$ phasor current variables, and $2 H$ independent (linear or nonlinear) equations that can be written such that there is one independent and one dependent variable at each harmonic $h$.

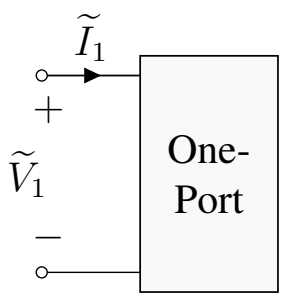

Figure 3.2: General one-port network.

\subsubsection{Defining Network Topologies}

A distribution network can be viewed as a set of subgraphs formed by interconnecting network ports with ideal interconnects; this is the framework of how devices and distribution systems are 
modeled in this research. In this approach, each subgraph links at least two network ports, each network port forms an "edge" in the subgraph. The subgraphs are related (coupled) to each other indirectly via the relationships defined by the one and two-port networks.

Figure 3.3 demonstrates an example of an electrical network described in this way. The network depicted in Figure 3.3 could represent an ideal voltage source (device $d=1$ ) connected to a transformer $(d=2)$ that in turn feeds two rectifiers $(d=3, d=4)$ serving resistive loads $(d=5$, $d=6)$.

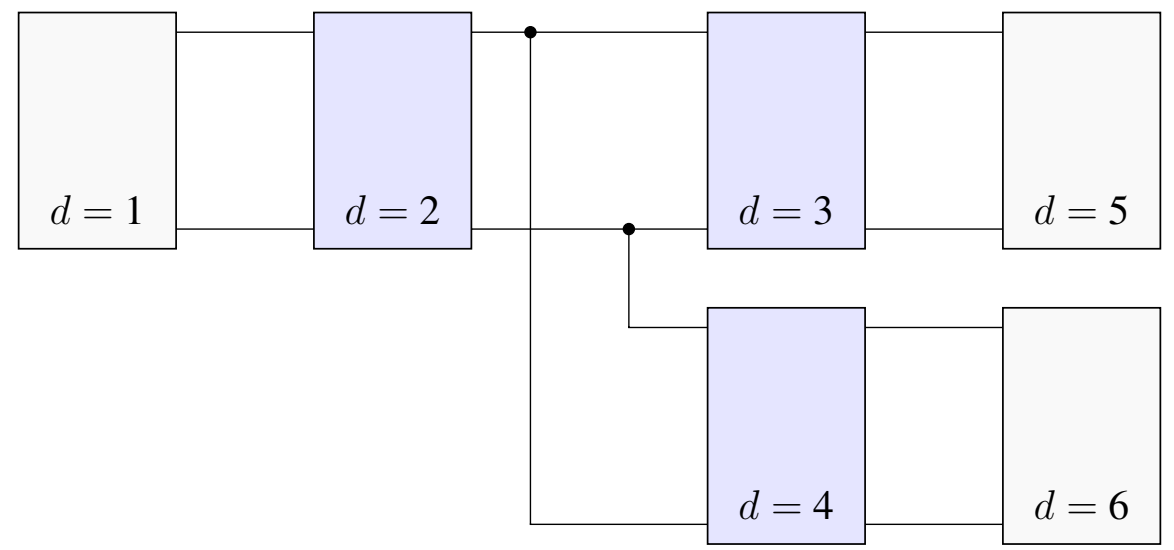

Figure 3.3: Example electrical network containing interconnected devices, wherein each device is a one- or two-port network.

Figure 3.4 is a directed graph representation of the network of Figure 3.3, demonstrating how the overall network can be decomposed into four directed subgraphs linked indirectly via the twoport network equations for devices $d=2$ (subgraphs $g=1$ and $g=2$ ), $d=3$ (subgraphs $g=2$ and $g=3$ ), and $d=4$ (subgraphs $g=2$ and $g=4$ ). In Figure $3.4, n_{g, p}$ represents port $p$ of subgraph $g$ and $e_{g, k}$, represents edge $k$ of subgraph $g$.

In an electrical network, the total number of variables is twice the number of edges (branches): one voltage and one current per edge [47]. Let $E$ represent the total number of edges; the number of variables is then $2 E$. (Note for simplicity, harmonics are not yet being considered; see below for treatment of harmonics.) Not that the number of edges $E$, is also equivalent to the number of ports defined by the devices, since each edge is also a port. That is, $E=V+2 W$, where $V=|\mathcal{V}|$ 


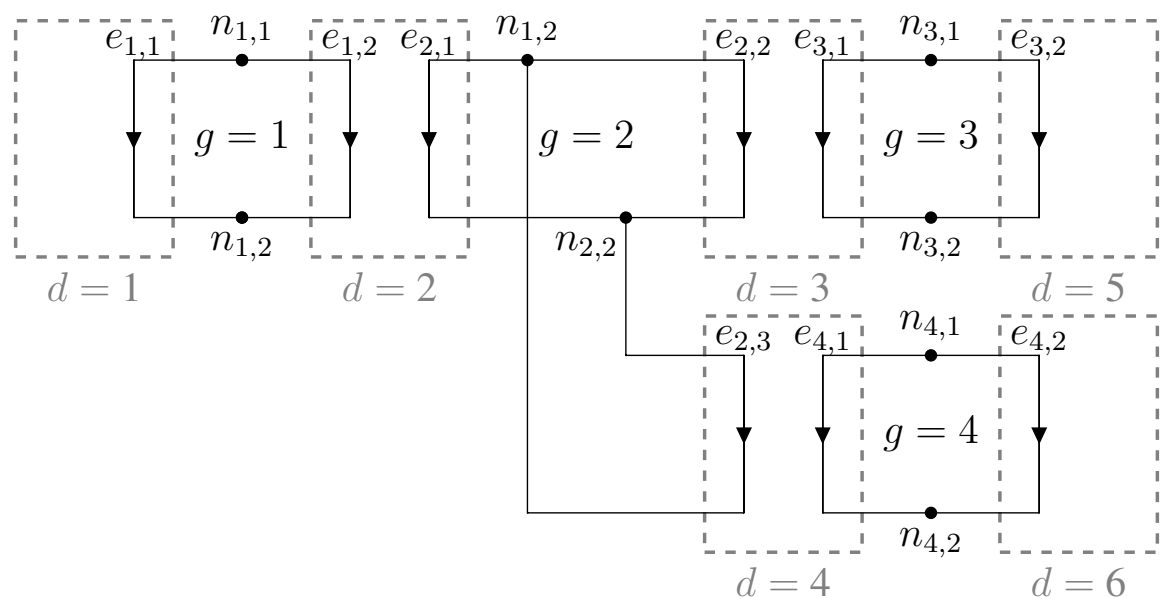

Figure 3.4: Directed graph representation of the electrical network of Figure 3.3.

and $W=|\mathcal{W}|$. Assuming that the system has a unique solution, $2 E$ independent equations must be defined to represent the system.

For any given subgraph $g$, there exists $E_{g}-N_{g}+1$ independent Kirchhoff's Voltage Law (KVL) equations and $N_{g}-1$ independent Kirchhoff's Current Law (KCL) equations, for a total of $E_{g}$ linear KVL and KCL equations. For all subgraphs (i.e., the network as a whole), there are therefore $E$ linear KVL and KCL equations. To fully determine the system, another $E$ equations are needed; these are obtained from the "connection equations" defined from the two-port networks. Since $E=V+2 W$, the $2 E$ equations are thus obtained.

However, note that is not typically necessary to obtain all $2 E$ equations to fully solve a system. For any given subgraph, the circuit can be fully represented by $N_{g}-1$ node voltages or $E_{g}-N_{g}+1$ loop currents. This means that the entire network could be represented by $N-G$ node voltage state variables (for instance); the remaining voltages could be derived via KVL and the currents then solved via the connection equations and KCL. In practice, what this means is that the system of equations can generally be reduced to a smaller system of equations via substitution.

When considering harmonics, there are $2 E H$ total variables, $E H$ total equations from KVL and KCL, and $V H+2 W H=E H$ variables from the one- and two-port network connection equations. Using the node voltage phasors as state variables, a carefully selected set of $(N-G) H$ equations involving those voltages could be used to define the system. 
To illustrate the concepts in the foregoing, suppose the circuit in Figure 3.3 is to be solved for the set of $H=3$ harmonics, $h \in 1,3,5$. As shown in Figure 3.4, the system has $E=9$ edges across the four subgraphs, for a total of $2 E H=54$ variables. There are $V=3$ one-port and $W=2$ two-port devices in the system. The required number of equations to solve the system is obtained from as follows:

- $5 H=15 \mathrm{KVL}$ equations; $H$ from subgraph $g=1,2 H$ from subgraph $g=2, H$ from subgraph $g=3$, and $H$ from subgraph $g=4$;

- $4 H=12 \mathrm{KCL}$ equations; $H$ from each of the for subgraphs;

- $V H=9$ connection equations from one-port devices;

- $2 W H=18$ connection equations from two-port devices.

The total number of equations obtainable from the network is therefore 54. However, note that by defining $(N-G) H=(8-4) 3=12$ node voltage phasors, a solution to the network states could theoretically be obtained. Therefore, in this example, the number of simultaneous equations needed to solve the system is no more than 54 and could be as few as 12 (using variable substitution). Later in the dissertation, chapter 6 describes the modeling framework implemented in a modeling language called Modelica. Such trivial equations are automatically eliminated by the language compiler to generate a consistent set of equations.

\subsection{Harmonic Power Flow Solution}

Solution of a set of generally nonlinear equations representing an arbitrary network is accomplished in the modeling toolkit by employing the harmonic power flow (HPF) approach. The HPF method extends the traditional power flow methodology in three key aspects:

1. Representation of additional harmonic frequencies beyond the fundamental (line) frequency in the system state; 
2. Representation of the nonlinear behavior of all devices in the system, including individual harmonic contribution and potential cross-coupling between harmonics;

3. Enforcement of power balance across all harmonics [3] [24].

In networks containing power electronic converters or other nonlinear circuit elements, current into nonlinear device $d$ at port $p=1$, and harmonic $h$ is specified in the HPF method by a generally nonlinear complex-valued function:

$$
\widetilde{I}_{d, 1, h}=g_{d}\left(h, P_{\mathrm{in}, d, 1}, \widetilde{\boldsymbol{I}}_{d, 1}, \widetilde{\boldsymbol{V}}_{d, 1} ; \boldsymbol{\gamma}_{d}\right), \quad d \in \mathcal{D}_{n, \ell}
$$

where $\mathcal{D}_{n, \ell} \in \mathcal{D}$ is the set of all nonlinear devices in the system; $P_{\mathrm{in}, d, 1}$ is the average real power into the device $d$, at harmonics $h \in \mathcal{H}$ computed as:

$$
P_{\mathrm{in}, d, 1}=\sum_{h \in \mathcal{H}} \mathbb{R e}\left\{\widetilde{V}_{d, 1, h} \widetilde{I}_{d, 1, h}^{*}\right\}
$$

where $\widetilde{V}_{d, 1, h}$ is the voltage and $\widetilde{I}_{d, 1, h}$ is the input current of device $d$, port 1 and harmonic $h$, respectively; $\widetilde{\boldsymbol{I}}_{d, 1}$ in (3.9) is a vector of phasor currents into port $p=1$ at device $d$, at harmonics $\mathcal{H} \backslash\{h\}$, in addition to possibly other harmonic current phasors in the network:

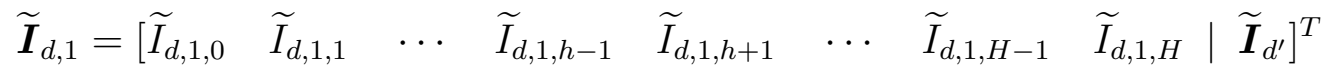

where $\widetilde{I}_{d, 1, h}, h^{\prime} \in \mathcal{H} \backslash\{h\}$ is the device $d$ input current at harmonic $h^{\prime}$ and $\widetilde{\boldsymbol{I}}_{d^{\prime}}$ is a vector of currents in the network that do not include any currents in the top portion of the vector in (3.11), or $\widetilde{I}_{d, 1, h} ; \widetilde{\boldsymbol{V}}_{d, 1}$ in (3.9) is a set of voltage phasors including at least the voltage at device $d$, port 1 , and harmonic $h$, but possibly others in the network:

$$
\widetilde{\boldsymbol{V}}_{d, 1}=\left[\begin{array}{lll}
\widetilde{V}_{d, 1, h} & \mid \tilde{\boldsymbol{V}}_{d^{\prime}}
\end{array}\right]^{T}
$$


where $\widetilde{V}_{d, 1, h}$ is the device $d$, port 1 voltage at harmonic $h$ and $\widetilde{\boldsymbol{V}}_{d^{\prime}}$ is a vector of voltages in the network other than $\widetilde{V}_{d, 1, h}$; finally the vector $\gamma_{d}$ in (3.9) contains the device-specific behavioral parameters. The interpretation of (3.9) is that the $h$ 'th harmonic of the current phasor into the device $d$, at port $p=1$, is generally a function of: (a) the harmonic number $h$ itself, (b) the device's real input power, (c) currents into the device at other harmonics $(\neq h)$, and possibly other currents in the network at any harmonic (d) voltage harmonics at port $p=1$ of the device, and possibly other nodes in the network, and (e) device-specific parameters, e.g., control settings, physical parameters, device loading.

Solution of the network equations using HPF is accomplished using an iterative numerical procedure (e.g., Newton Raphson) to obtain convergence of all states at all specified harmonics within a specified threshold, while explicitly enforcing power balance throughout the network. In particular, the method includes the following steps:

1. Initialize all network voltages and currents. For example, set the magnitudes and phase angles of all voltage and current phasors to zero, except known phasor voltage at ports of voltage input source(s).

2. Compute network currents. Using the last converged solution of network voltage phasors, compute current phasors through all branches of the network using either linear circuit analysis or (3.9) for all linear and nonlinear devices, respectively, in the network.

3. Enforce power balance. For each nonlinear device, compute the average real power input into the device, $P_{\text {in }, d}$. Set or compute (if not known) the average real output power $\left(P_{\text {out }, d}\right)$ of the device. Using the loss function for the device (see section 4.4), adjust the magnitude of the current phasors at the input port of the device so that the power balance is achieved.

4. Update network voltages. Using the adjusted currents in step 3, update all node voltage phasors in the network.

5. If not converged, return to step 2 . 
Next chapter describes how the network devices are modeled, including how nonlinear device behavior and losses are represented in the modeling framework. 


\section{Chapter 4}

\section{Device Models}

This section illustrates example linear and nonlinear device models for specific component types, focusing on those used in the validation studies in section 5. In each case, the models below define $2 \mathrm{H}$ connection equations for each device; exactly two of the four voltage and current phasors at each harmonic can be considered independent, while the other two are dependent.

\subsection{Series and Shunt Impedances}

\subsubsection{Series Impedance}

Consider a series impedance component, with impedance $Z(h)=R+j h X$, where $R$ is the resistance and $X$ is the reactance at the fundamental $(h=1)$ frequency harmonic. Using the system definitions from previous chapter, the following relationships apply:

$$
\begin{aligned}
\widetilde{I}_{d, 1, h} & =-\widetilde{I}_{d, 2, h} \\
\widetilde{V}_{d, 1, h} & =\widetilde{V}_{d, 2, h}-Z(h) \widetilde{I}_{d, 2, h}
\end{aligned}
$$

The $A B C D$ matrix of the series component for any harmonic is therefore:

$$
\left[\begin{array}{c}
\widetilde{V}_{d, 1, h} \\
\widetilde{I}_{d, 1, h}
\end{array}\right]=\left[\begin{array}{cc}
1 & Z(h) \\
0 & 1
\end{array}\right]\left[\begin{array}{c}
\widetilde{V}_{d, 2, h} \\
-\widetilde{I}_{d, 2, h}
\end{array}\right]
$$




\section{Shunt Impedance}

In a shunt impedance, the component is in parallel, therefore the terminal voltages are equal. On the contrary, the current is divided. The network equations are:

$$
\begin{aligned}
\widetilde{I}_{d, 1, h} & =\frac{\widetilde{V}_{d, 2, h}}{Z(h)}-\widetilde{I}_{d, 2, h} \\
\widetilde{V}_{d, 1, h} & =\widetilde{V}_{d, 2, h}
\end{aligned}
$$

and the $A B C D$ matrix of the shunt impedance for any harmonic is:

$$
\left[\begin{array}{c}
\widetilde{V}_{d, 1, h} \\
\widetilde{I}_{d, 1, h}
\end{array}\right]=\left[\begin{array}{cc}
1 & 0 \\
\frac{1}{Z(h)} & 1
\end{array}\right]\left[\begin{array}{c}
\widetilde{V}_{d, 2, h} \\
-\widetilde{I}_{d, 2, h}
\end{array}\right]
$$

Note that the equation for each harmonic in (4.2) and (4.4) are decoupled, and the total number of equations is $2 H$.

\subsection{Constant Power Loads}

Constant power loads (CPLs) are modeled as one-port components that absorb a fixed amount of real and reactive power to enforce the relation:

$$
\widetilde{V}_{d, 1, h}=\frac{\widetilde{S}_{\mathrm{in}, d, 1, h}}{\widetilde{I}_{d, 1, h}^{*}}
$$

where $\widetilde{S}_{\mathrm{in}, d, 1, h}=P_{\mathrm{in}, d, 1, h}+j Q_{\mathrm{in}, d, 1, h}$ is the total input harmonic apparent power, $P_{\mathrm{in}, d, 1}$ is the total real power into the device given in (3.10), and

$$
Q_{\mathrm{in}, d, 1}=\sum_{h \in \mathcal{H}} \operatorname{Im}\left\{\widetilde{V}_{d, 1, h} \widetilde{I}_{d, 1, h}^{*}\right\}
$$


is the total reactive power into device $d$, port $p=1$. In the toolkit, users specify the components of the real and reactive harmonic power contributions in the summations in (3.10)-(4.6).

\subsection{Transformers}

\subsubsection{Single-phase Transformer}

Single-phase transformers can be modeled as two-port devices. The most general representation of the single-phase transformer, allowing for nonlinear effects such as magnetic saturation, would require a general nonlinear two-port network model, as discussed in chapter 3 . In cases where nonlinear effects can be neglected, in addition to winding and magnetic core impedances, the ideal transformer model with voltage ration $N_{1} / N_{2}$ (primary winding turns : secondary winding turns) shown in Figure 4.1 is used.

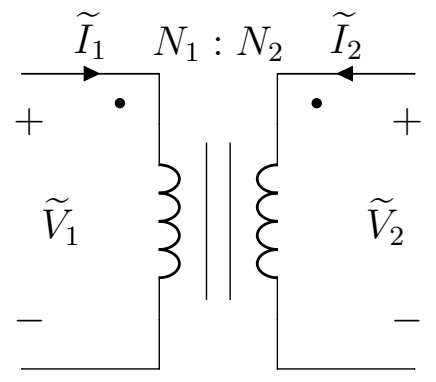

Figure 4.1: Ideal single-phase transformer model.

The $A B C D$ matrix representation for this ideal transformer component at any harmonic is:

$$
\left[\begin{array}{c}
\widetilde{V}_{d, 1, h} \\
\widetilde{I}_{d, 1, h}
\end{array}\right]=\left[\begin{array}{cc}
a & 0 \\
0 & \frac{1}{a}
\end{array}\right]\left[\begin{array}{c}
\widetilde{V}_{d, 2, h} \\
-\widetilde{I}_{d, 2, h}
\end{array}\right]
$$

where $a:=N_{1} / N_{2}$. A high-fidelity model of the single-phase transformer which includes winding and magnetization impedances, but neglects nonlinear effects, is shown in Figure 4.2. Note that the transformer model in Figure 4.2 is a composite model, utilizing series and shunt impedance elements and the ideal transformer. 


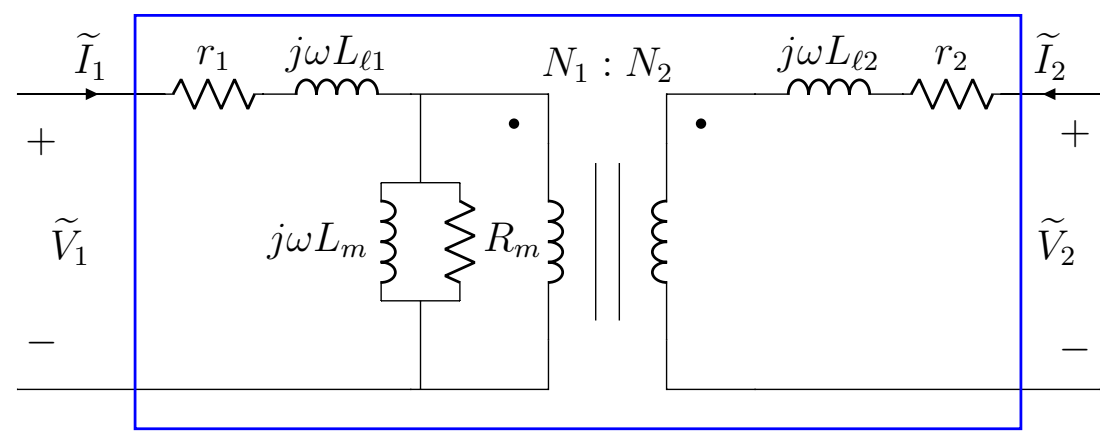

Figure 4.2: Equivalent steady-state circuit for single-phase transformer. The blue box defines a two-port component.

In Figure 4.2, $r_{1}, r_{2}$ are the resistances of winding 1 and 2 respectively; $L_{\ell 1}, L_{\ell 2}$ are the leakage inductances of winding 1 and 2 respectively; $L_{m}, R_{m}$ are the magnetization inductance and resistance of the core respectively; $N_{1}, N_{2}$ are the number of turns of winding 1 and 2 respectively; $\omega$ is the fundamental radial electrical frequency of the input. The internal circuit with boundary defined by the blue box in Figure 4.2, represents a linear two-port network. Using the definitions of series, shunt impedance and the ideal transformer device model equations, one can simplify the system to the following $A B C D$ matrix valid for any harmonic:

$$
\left[\begin{array}{c}
\widetilde{V}_{d, 1, h} \\
\widetilde{I}_{d, 1, h}
\end{array}\right]=\left[\begin{array}{cc}
a\left(\frac{Z_{1}(h)+Z_{3}(h)}{Z_{3}(h)}\right) & \left(\frac{1}{a}\right)\left(\frac{\left(a^{2} Z_{2}(h)+Z_{3}(h)\right)\left(Z_{1}(h)+Z_{3}(h)\right)-Z_{3}^{2}(h)}{Z_{3}(h)}\right) \\
a\left(\frac{1}{Z_{3}(h)}\right) & \left(\frac{1}{a}\right)\left(\frac{a^{2} Z_{2}(h)+Z_{3}(h)}{Z_{3}(h)}\right)
\end{array}\right]\left[\begin{array}{c}
\widetilde{V}_{d, 2, h} \\
-\widetilde{I}_{d, 2, h}
\end{array}\right]
$$

where, $Z_{1}(h):=r_{1}+j \omega h L_{\ell 1}, Z_{2}(h):=r_{2}+j \omega h L_{\ell 2}, Z_{m}(h):=R_{m} \| j \omega h L_{m}$.

\subsubsection{Three-phase Transformers}

Three-phase transformers can be viewed as multiple-port devices, depicted in Figure 4.3. The most general representation of the device would include nonlinear effects such as magnetic saturation, differences in electrical parameters as a result of practical considerations of windings in actual transformers, and voltage imbalances; these non-idealities would generally lead to cross-coupling between harmonic between all phases, and would require the use of general nonlinear two-port 
network model. However, under certain assumptions (see below) it is possible to represent the device as a set of linear and uncoupled two-port devices.

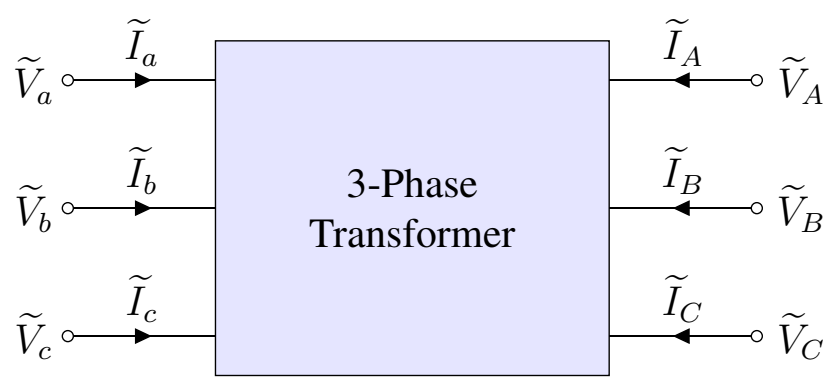

Figure 4.3: Three-phase transformer component.

Suppose that the voltages and currents on the right side (analogous to a "port") of the component in Figure 4.3 are known, and the voltages and currents on the left side are unknown; six equations are therefore needed to solve for the unknowns. Assuming the device is operating in the linear magnetic region (i.e., not magnetically saturated), there is a symmetry of electrical transformer parameters among phases, and the three-phase voltage and current are balanced, each electrical phase of the transformer can be represented in the steady-state as three separate circuits, with two-port transformer models as shown in Figure 4.2. In this way, two of the unknown variables can be solved in each of the three circuits, yielding the required six unknowns. An example twoand one-port representation of a balanced network illustrating this concept is shown in Figure 4.4.

In Figure 4.4, the network represents a three-phase utility voltage supplying a $\Delta$-Y connected building transformer with balanced loads across its three output windings. In this case, devices $d=\{1,4,5\}$ are represented by the two-port transformer models shown in Figure 4.2; devices $d=\{2,5,8\}$ and $d=\{3,6,9\}$ are two- and one-port devices, respectively. The numbering convention of voltages and currents is assigned arbitrarily; in this case, $\widetilde{V}_{1}, \widetilde{I}_{1}$ and $\widetilde{V}_{2}, \widetilde{I}_{2}$ are the voltage and current inputs (outputs) of the electrical $a$-phase transformer winding. Figure 4.4 also illustrates how network connects can result in a reduced number of system unknowns, as discussed in section 3.2.2; for example, in this case, the output current $\widetilde{I}_{2}$ of device $d=1$ is the (negative) input current into device $d=2$. 


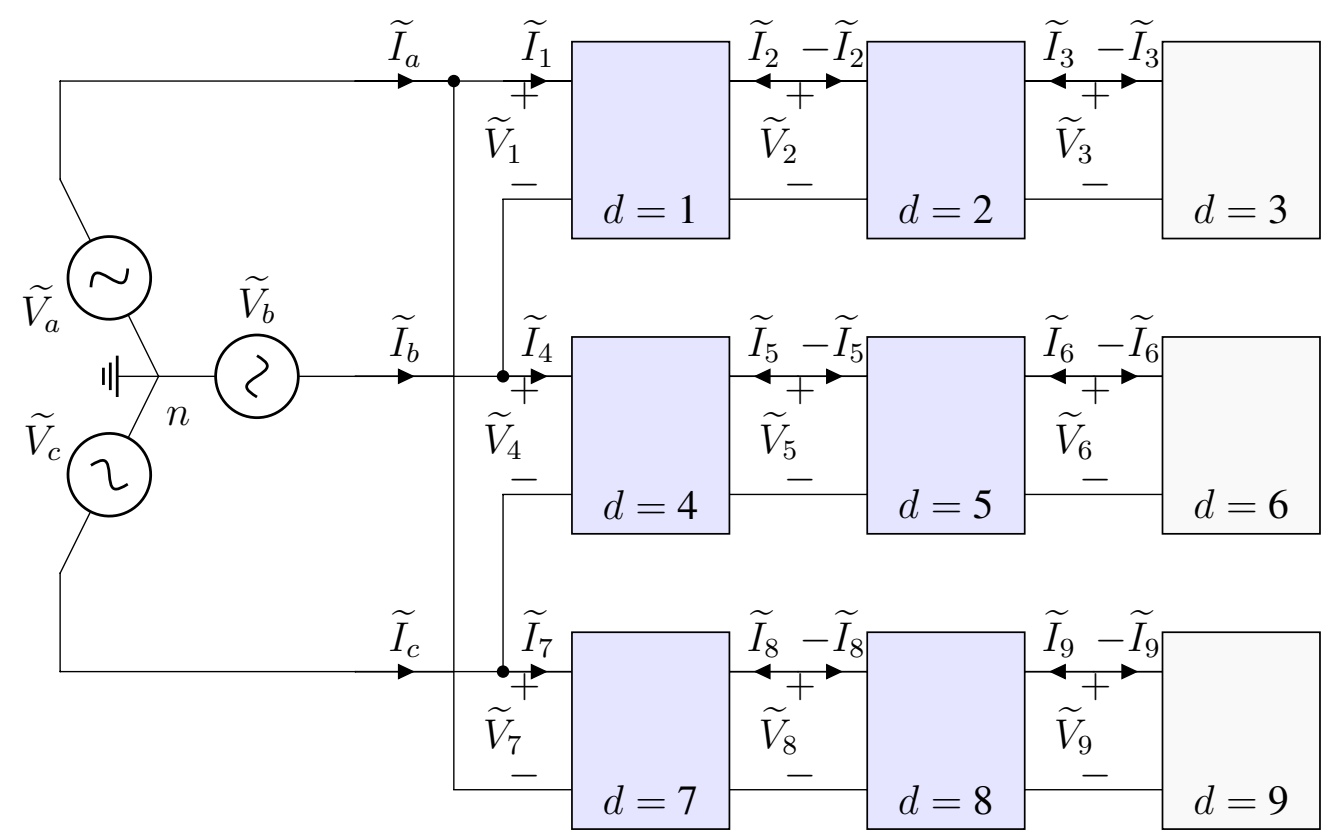

Figure 4.4: Balanced electrical network with two-port linear transformer models.

If nonlinear effects are neglected, but it cannot be assumed that the transformer parameters are electrically symmetric and/or there are voltage imbalances, then there will generally be coupling of harmonics between phases. In this case, the transformer can be represented by modifications of the general two-port network matrix in (4.8) must both be augmented to include variables for the additional (third) winding on each side of the transformer.

\subsection{Power-electronic Converters}

Power-electronic converters are generally nonlinear devices, requiring the use of a generalized two port nonlinear component model. The following sections describe the nonlinear device behavior, loss representation and power balance for converter components.

\subsubsection{Converter Device-Specific Behavior}

The parameterized, device-specific behavior function $g_{d}$ in (3.9) is a generally nonlinear function which describes the behavior of the input current through device $d$, as a function of harmonic 
number, the device input voltage and power, and possibly other bus current and voltage harmonics in the system. The vector $\gamma_{d}$ contains parameter information specific to the device.

Several approaches can be used to determine the form and best-fit parameters for $g_{d}$ so that it can be used in HPF algorithm, subject to the restriction that $g_{d} \in \mathcal{C}^{2}$. The first step is to determine the general form of the function from measured or simulated time-domain device behavior, under a range of device input or output powers. Typically, observation readily indicates which variables most impact magnitude and phase angle. Proceeding further, one may use several general approaches to functionally describe the behavior, e.g.:

1. Empirical Method: Using measured or simulated current waveforms, express $g_{d}$ as an $n$-dimensional surface, where $n$ is the number of variables determined heuristically to influence current behavior. Use linear interpolation between measured (simulated) points; note that linear representation of the function ensures $g_{m} \in \mathcal{C}^{2}$.

2. Analytical Method: Again, using measured (simulated) current waveforms, express $g_{d}$ as a closed-form function in $\mathcal{C}^{2}$, in terms of an unknown parameter set. Using a nonlinear numerical optimization method, determine the best-fit parameters which minimize a distance metric of the error between predicted and measured (simulated) current magnitudes and phase angles ( [48]).

In the validation studies described ahead in chapter 8 , the empirical method was used to determine $g_{d}$. Therein, experimental observation indicated that the current magnitude $\left\|\widetilde{I}_{d, 1, h}\right\|$ for the device under study were most influenced by the harmonic number $h$ and device input power $P_{\text {in }, d, 1}$. Figures 4.5 and 4.6 show surface plots of the current magnitude and phase angle, respectively, of a power-electronic converter (Power Supply 3, see section chapter 8).

As shown in Figure 4.5 the magnitude of the measured current harmonics can be described as a generally smooth and "well-behaved" function of harmonic number itself and input power. Figure 4.6, indicates that the current phase angle is generally a function of harmonic number only. Chapter 7 is dedicated to the harmonic behavior models for nonlinear converters. It describes proposed models for DBFs and a parameter estimation procedure for the models. 


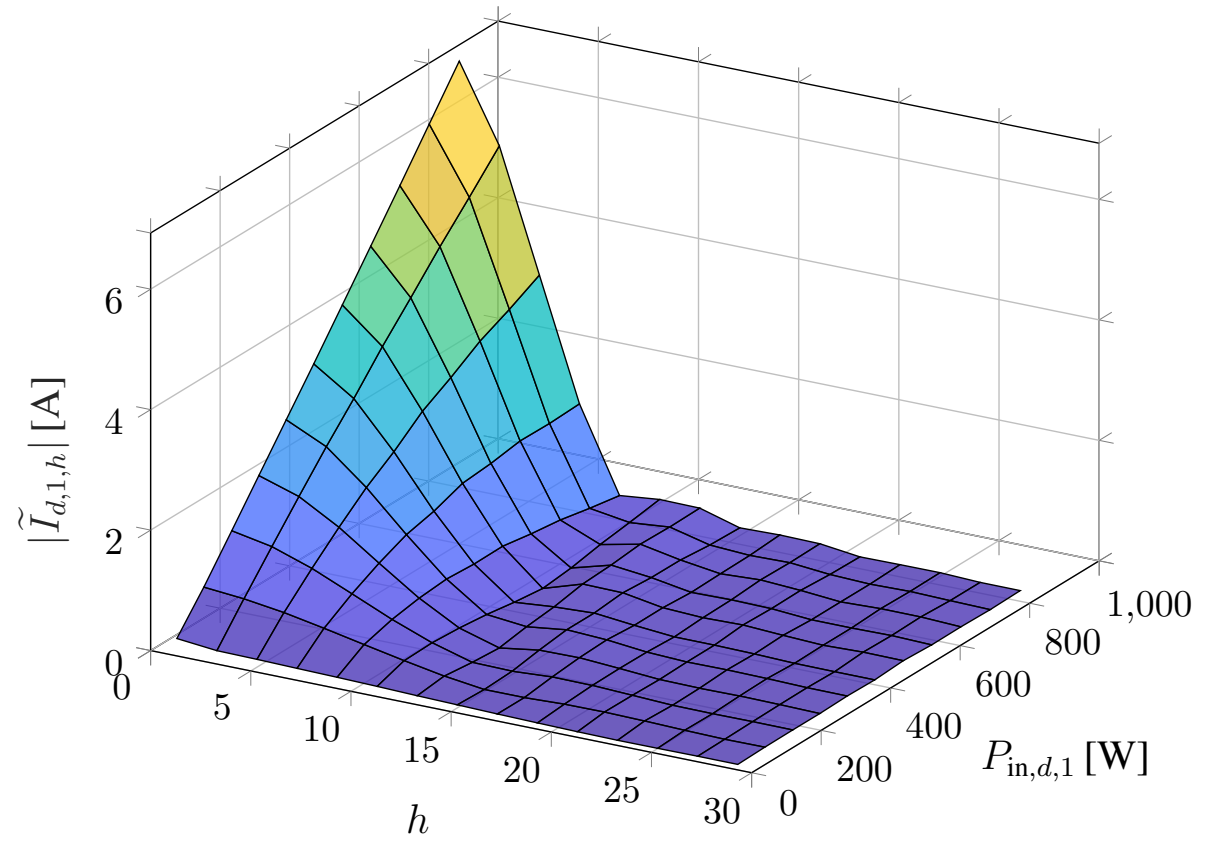

Figure 4.5: Surface plot of harmonic current magnitude variation with harmonic number and real power at $h=1$.

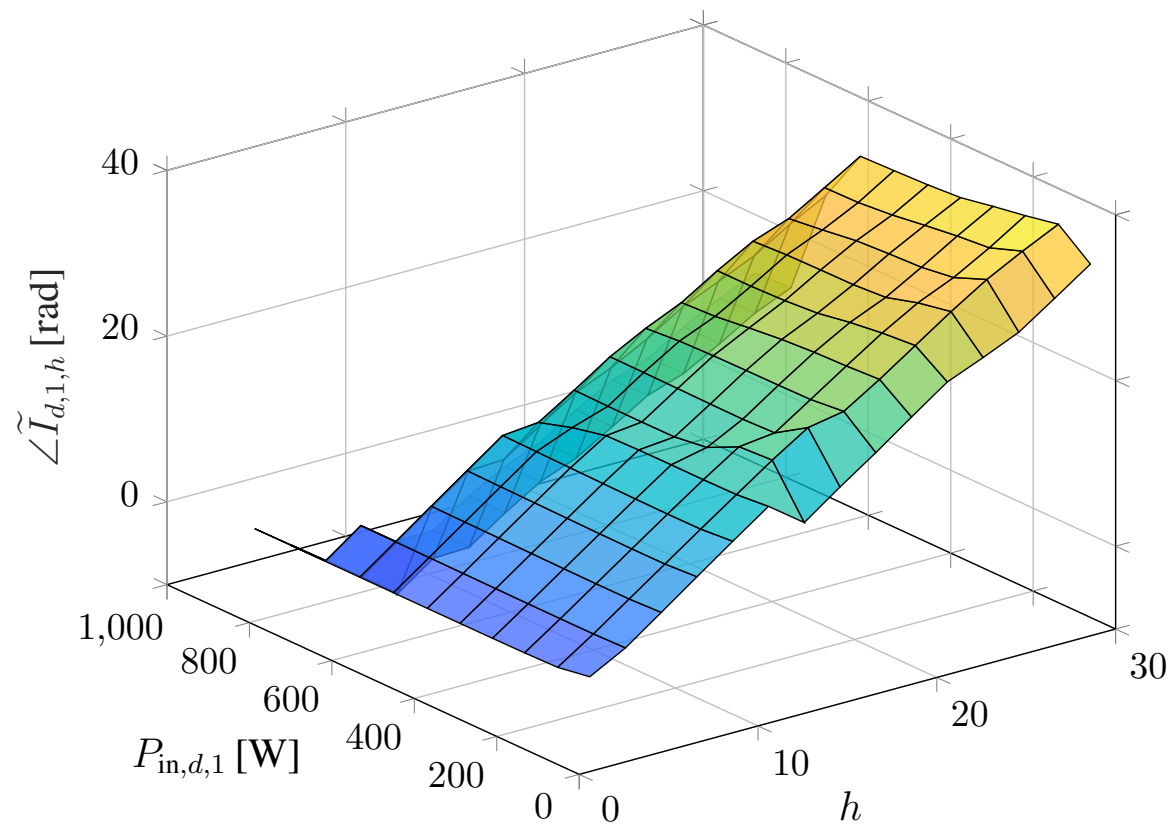

Figure 4.6: Surface plot of harmonic current phase angle variation with harmonic number and real power at $h=1$. 


\subsubsection{Converter Loss Modeling}

To enforce power balance for nonlinear devices in the network, it is necessary to model and characterize device losses. The average real power into device $d$, measured at port $p=1$, is given in (3.10). The average real power out of device $d$, measured at port $p=2$, denoted $P_{\text {out }, d, 2}$ is given as:

$$
P_{\text {out }, d, 2}=-\sum_{h \in \mathcal{H}} \mathbb{R e}\left\{\widetilde{V}_{d, 2, h} \widetilde{I}_{d, 2, h}^{*}\right\}
$$

The relationship between average real input power, output power and power losses used herein expands upon the work in [49] [50] and is expresses in the form:

$$
\begin{aligned}
P_{\text {in }, d, 1} & =P_{\text {out }, d, 2}+\overbrace{\left(\beta_{1}+\beta_{2} P_{\text {out }, d, 2}+\beta_{3} P_{\text {out }, d, 2}^{2}\right)}^{P_{\text {loss }, d}} \\
& =P_{\text {out }, d, 2}+f_{\ell}\left(P_{\text {out }, d, 2} ; \boldsymbol{\beta}_{d}\right)
\end{aligned}
$$

where $P_{\text {loss }, d}$ is the average real power loss in the device, $f_{\ell}(\cdot)$ is the loss function, and vector $\boldsymbol{\beta}_{d}$ contains parameters for the device $\beta_{1 d, i} \in \mathbb{R}, i \in\{1,2,3\}$.

Use of (4.10) with fitted parameters has been shown to yield reasonable accuracy when compared to measurements for a variety of devices [49] [50]. However, the model suffers from the drawback of not adequately representing the power relationship at low power levels for some devices; for example, this discrepancy can arise if the device enters a "stand-by" or power savingmode when the converter is operating at no load. This is observed in high power rated power supplies that might have a more sophisticated control circuit. To account for this behavior, this research proposes a "two-state"power loss function expressed as:

$$
f_{\ell, 2 s}=\lambda P_{\mathrm{sb}, d}+(1-\lambda) f_{\ell}\left(P_{\mathrm{out}, d, 2} ; \overline{\boldsymbol{\beta}}\right)
$$

where $P_{\mathrm{sb}, d}$ is the stand-by power of device $d$, scaling parameter $\lambda$ is defined as: 


$$
\lambda:=\left\{\begin{array}{cl}
\frac{\min \left(P_{\mathrm{out}, d, 2}\right)-P_{\mathrm{out}, d, 2}}{\min \left(P_{\mathrm{out}, d, 2}\right)}, & P_{\mathrm{out}, d, 2} \leq \min \left(P_{\mathrm{out}, d, 2}\right) \\
0, & P_{\mathrm{out}, d, 2}>\min \left(P_{\mathrm{out}, d, 2}\right)
\end{array}\right.
$$

where $\min \left(P_{\text {out }, d, 2}\right)$ is the minimum measured output power of the device when it is not oper-

ating in stand-by mode; vector $\overline{\boldsymbol{\beta}}_{d}$ are the fitted loss parameters for the function $f_{\ell}$ in (4.10) after removing measured powers less than $\min \left(P_{\text {out }, d, 2}\right)$. Assigning $\beta_{d, 0}=\min \left(P_{\text {out }, d, 2}\right)$ and collecting parameters in vector $\boldsymbol{\beta}_{d}^{\prime}=\left[\beta_{d, 0} \mid \overline{\boldsymbol{\beta}}_{d}\right]^{T}$, the power relationship for the nonlinear devices is expressed as:

$$
\begin{aligned}
P_{\mathrm{in}, d, 1} & =P_{\mathrm{out}, d, 2}+f_{\ell, 2 s}\left(P_{\mathrm{out}, d, 2} ; \boldsymbol{\beta}_{d}^{\prime}\right) \\
& =f\left(P_{\mathrm{out}, d, 2} ; \boldsymbol{\beta}_{d}^{\prime}\right)
\end{aligned}
$$

Herein, $P_{\mathrm{in}, d, 1}=f\left(P_{\mathrm{out}, d, 2} ; \boldsymbol{\beta}_{d}^{\prime}\right)$ denotes the modified two-state power relationship, with adjusted loss parameters $\boldsymbol{\beta}_{d}^{\prime}$, for all devices $d \in \mathcal{D}_{n \ell}$. Identification of the best-fit loss parameters $\beta_{d, i}$ can be determined using a numerical fitting procedure to minimize the error between the measured and predicted power loss according to (4.12). An example curve for an AC/DC converter using linear-least squares is shown in Figure 4.7.

As shown in Figure 4.7, power loss measurements (black dots) at output powers less than $\min \left(P_{\text {out }, d, 2}\right)=100[\mathrm{~W}]$ do not fall along the same polynomial curve as measurements at higher power levels. Therefore, the curve obtained from $f_{\ell}$ in (4.10) using best-fit parameters with measurement points (red dashed line) results in noticeable discrepancy at low power levels. On the other hand, using the fitted curve $f_{\ell, 2 s}$ in (4.10), the error in the modeled loss is improved, particularly at lower power levels.

\subsubsection{AC/DC Converters}

To model AC/DC converters ("rectifiers"), power balance and internal loss modeling were calculated in this research using (3.11) and (3.10)-(4.6). Since these are nonlinear devices, harmonic currents on the input side of each component (designated as port $p=1$ below) are computed from 


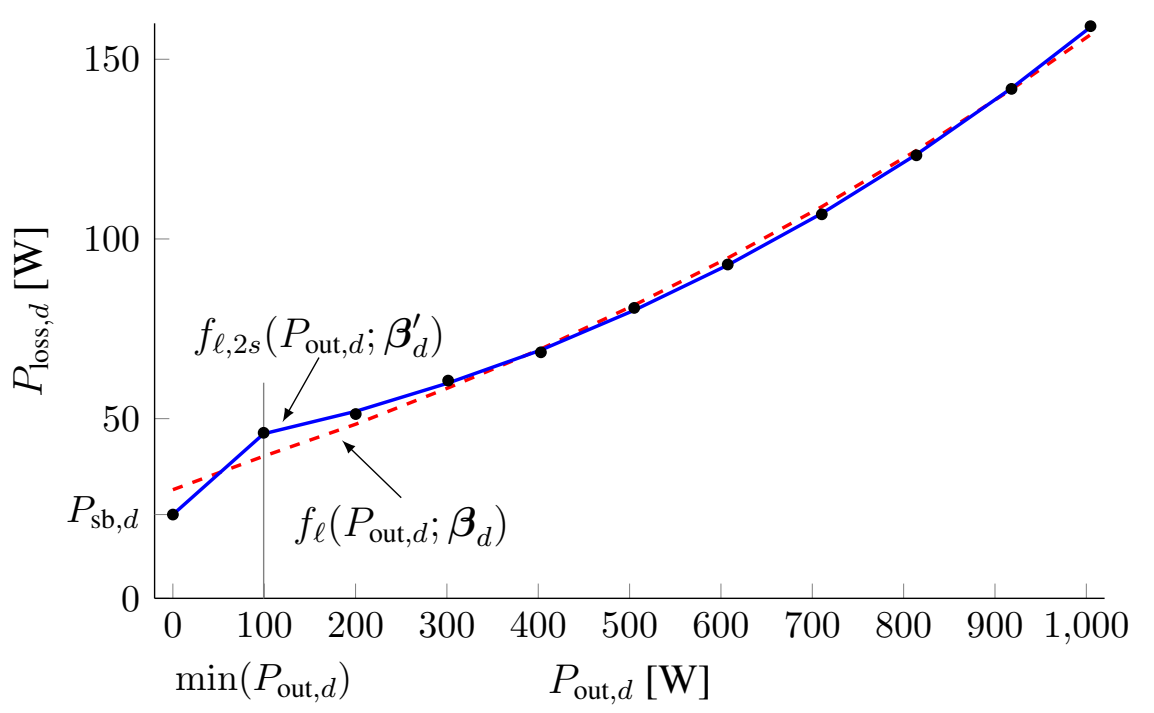

Figure 4.7: Comparison of two-state loss model (blue, solid) to uncorrected loss model (dashed, red); power loss measurements are shown as black dots.

(3.9). In the validation studies shown later in chapter 8, a simplified form of (3.9) was used, i.e., $g_{d}$ was assumed to be parameterized function of harmonic number, output power, and fundamental input current only, which can be written as:

$$
\widetilde{I}_{d, 1, h}=g_{d}\left(h, P_{\mathrm{in}, d, 1}, \widetilde{\boldsymbol{I}}_{d, 1} ; \boldsymbol{\gamma}_{d}\right), \forall h>1
$$

In addition, there are three constrain equations for the rectifier: DC input current is zero $\left(\widetilde{I}_{d, 1,0}=\right)$, DC output voltage is regulated $\left(\widetilde{V}_{d, 2,0}=V_{D C}\right)$, and AC output voltage is zero $\left(\widetilde{V}_{d, 2, h}=\right.$ $0, \forall h>0)$. Thus, the rectifier component specifies on equation for power balance, $H-2$ equations for input current, one equation for the input DC current constraint, and $H$ equations for the output voltage constraints, yielding $2 H$ equations in total. Moreover, the equations have been specified such that for each harmonic $h$ there are exactly two independent variables and two dependent variables. 


\subsubsection{DC/DC Converters}

As with $\mathrm{AC} / \mathrm{DC}$ converters, port $p=1$ is designated as the input port and port $p=2$ as the output port. DC/DC converter losses are modeled using (4.10). In addition, DC/DC converters have the following constraints: AC input current in zero $\left(\widetilde{I}_{d, 1, h}=0, \forall h>0\right)$, DC output voltage is regulated $\left(\widetilde{V}_{d, 2,0}=V_{D C}\right)$, and AC output voltage is zero $\left(\widetilde{V}_{d, 2, h}=0, \forall h>0\right)$. This yields one equation for the $\mathrm{AC}$ input current constraints, and $H$ equations for the output voltage constraints, for a total of $2 H$ equations.

\subsubsection{DC/AC Converters}

DC/AC converters ("inverters") are modeled as voltage-source devices with a regulated (fixed) output voltage spectrum: $\widetilde{V}_{d, 2,0}=0$ and $\widetilde{V}_{d, 2, h}=\widetilde{V}_{A C, h}, \forall h>0$. (For a perfectly regulated inverter without switching harmonics, $\widetilde{V}_{d, 2,1}=\widetilde{V}_{A C, 1}$ and $\widetilde{V}_{d, 2, h}=0, h \neq 1$.) Inverter losses also follow (4.12). Finally, inverter AC input current is zero $\left(\widetilde{I}_{d, 1, h}=0, \forall h>0\right)$. This yields one equation for power balance, $H-1$ equations for the $\mathrm{AC}$ input current constraints, and $H$ equations for the output voltage constraints, again for a total of $2 H$ equations. 


\section{Chapter 5}

\section{Application of HPF on an Example Circuit}

The modeling concepts described in Chapter 3 are applied on an example AC electrical distribution system. Consider the system shown in Figure 5.1 that consists of an AC voltage source and a nonlinear load connected via a series impedance $Z_{1}$. An impedance $Z_{2}$ is acting as a terminal load impedance. The example is chosen as it is simple to implement and at the same time complex enough to capture and illustrate salient aspects of a Harmonic Power Flow formulation. Some of the salient features are: harmonic modeling of an AC electrical distribution system, nonlinear device modeling, Harmonic Power Flow in a fully coupled system, and port network modeling theory. The nonlinear load is modeled as an AC/DC converter that draws power $P_{\text {out }}$ (shown in the figure).

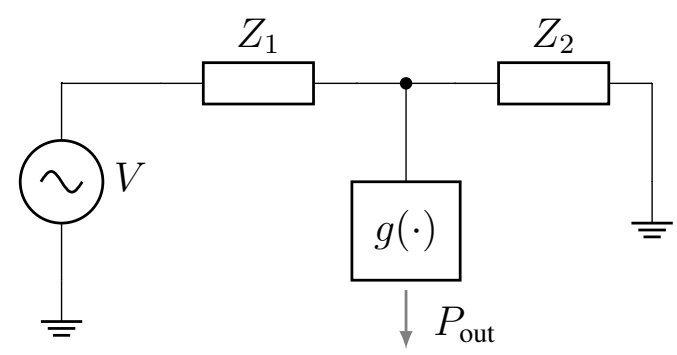

Figure 5.1: Example nonlinear network.

\subsection{Network Modeling}

Figure 5.2 shows the annotated form of the figure in 5.1. The devices are enumerated from left to right and top to bottom. The network consists of two subgraphs, $g=1$ and 2 . $Z_{1}$ is modeled as two-port series impedance, and $Z_{2}$ and nonlinear load $g(\cdot)$ is modeled as a one-port device. Given that the system has a total of $E=5$ edges, therefore, for a harmonic power flow formulation with $H$ harmonics, the system will need a total of $10 H$ equations to fully describe the system. 


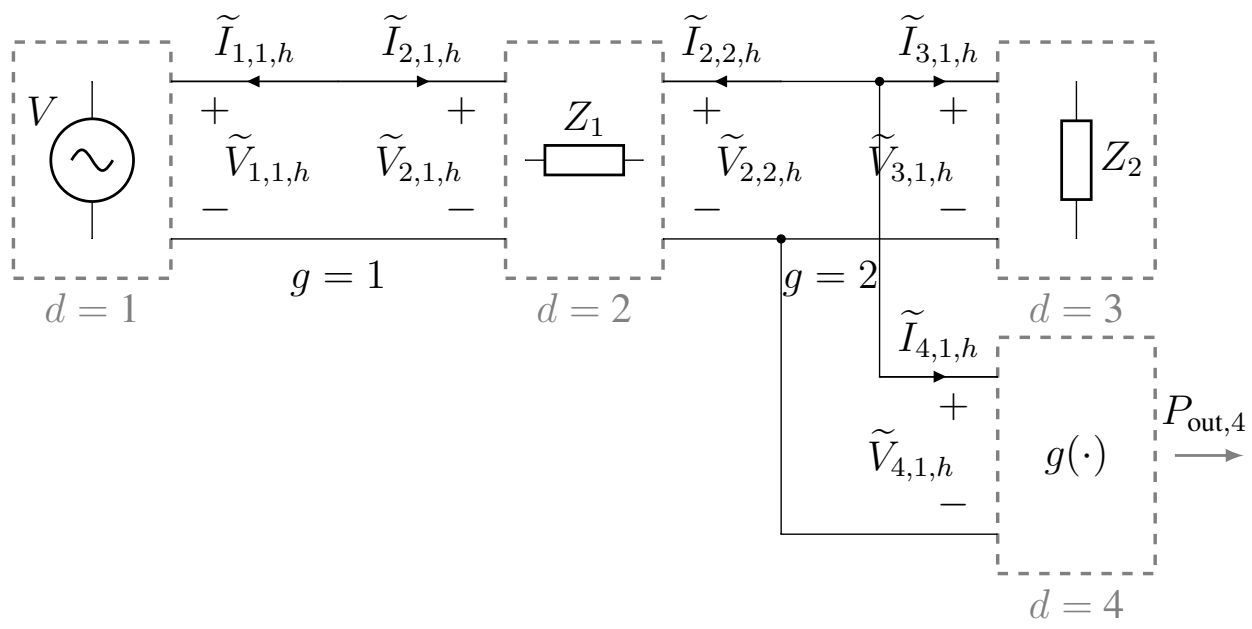

Figure 5.2: Example nonlinear network annotated using the network theory from Chapter 3 .

Writing KCL and KVL equations for all subgraphs. For subgraph $g=1$,

$$
\begin{aligned}
& \widetilde{V}_{1,1, h}=V_{2,1, h} \\
& \widetilde{I}_{1,1, h}=-\widetilde{I}_{2,1, h}
\end{aligned}
$$

For subgraph $g=2$,

$$
\begin{array}{lc}
\widetilde{V}_{2,2, h}= & V_{3,1, h} \\
\widetilde{V}_{4,1, h}= & V_{2,2, h} \\
\widetilde{I}_{2,2, h}= & -\widetilde{I}_{3,1, h}-\widetilde{I}_{4,1, h}
\end{array}
$$

Writing "connection" equations for the one- and two-port devices. Voltage source $V$ is an input source. The input to the voltage source is a complex valued vector $\widetilde{\boldsymbol{v}}=\left[\widetilde{v}_{1}, \ldots, \widetilde{v}_{H}\right], h \in \mathcal{H}$.

$$
\widetilde{V}_{1,1, h}=\widetilde{v}_{h}
$$


Device $d=2$ is the line impedance $Z_{1}$, therefore, using series impedance relation for a two port series impedance:

$$
\begin{aligned}
& \widetilde{V}_{2,2, h}=V_{2,1, h}-\widetilde{I}_{2,1, h} Z_{1}(h) \\
& \widetilde{I}_{2,1, h}=\quad-\widetilde{I}_{2,2, h}
\end{aligned}
$$

Impedance $Z_{2}$ (device $d=3$ ) is one-port impedance, therefore,

$$
\widetilde{V}_{3,1, h}=\widetilde{I}_{3,1, h} Z_{2}(h)
$$

Nonlinear harmonic load is modeled as a controlled current source. The device model is written as a complex valued function (see 4.4).

$$
\widetilde{I}_{4,1, h}=g_{4}\left(h, P_{\mathrm{in}, 4,1}, \widetilde{V}_{4,1, h}, \gamma_{4}\right)
$$

When combined with the KCL and KVL equations from above, it gives $10 H$ equations for $10 \mathrm{H}$ unknowns. Note that these equations are for the linear network. For a fully coupled harmonic power flow solution, we need to define a power balance equation for the nonlinear load. The power balance ensures that the total real power entering into the nonlinear load (real AC power drawn by the load) is equal to the sum of real power computed for all harmonics in the system, which is given as,

$$
P_{\mathrm{in}, 4,1,1}=f_{1}\left(P_{\mathrm{out}, 4} ; \boldsymbol{\alpha}_{1}\right)-\sum_{\forall h \in \mathcal{H} \backslash h_{1}} \widetilde{V}_{4,1, h} \widetilde{I}_{4,1, h}^{*}
$$

This adds an additional equation and an unknown variable $P_{\text {in }, 4,1,1}$, real power into device $d=4$, in port $p=1$, at fundamental frequency $h=1$ ( $1_{\text {st }}$ harmonic). The total equations are 
therefore $10 H+1$. These equations can be reduced by simple substitution and the final system equation are:

$$
\begin{array}{r}
Z_{1}(h) \widetilde{I}_{1,1, h}-\widetilde{V}_{4,1, h}+\widetilde{V}_{1,1, h}=q_{c 1}=0 \\
Z_{2}(h) \widetilde{I}_{3,1, h}-\widetilde{V}_{4,1, h}=q_{c 2}=0 \\
\widetilde{I}_{4,1, h}-g_{4}\left(h, P_{\mathrm{in}, 4,1}, \widetilde{V}_{4,1, h} ; \gamma\right)=q_{c 3}=0 \\
\widetilde{I}_{1,1, h}-\widetilde{I}_{3,1, h}-\widetilde{I}_{4,1, h}=q_{k 1}=0 \\
P_{\mathrm{in}, 4,1,1}+\sum_{\forall h \in \mathcal{H}_{\bar{p}}} \widetilde{V}_{4,1, h} \widetilde{I}_{4,1, h}^{*}-f_{1}\left(P_{\text {out }, d, 2} ; \boldsymbol{\alpha}_{1}\right)=q_{p 1}=0
\end{array}
$$

For simplicity, the nonlinear load is assumed to be lossless. Therefore, the output DC power drawn by a load is equal to input AC power, or, $P_{\mathrm{in}, 4,1}=P_{\mathrm{out}, 4,2}$. Once again, for simplicity, the nonlinear load device behavior is modeled as follows,

$$
\begin{array}{r}
g_{\|\cdot\|, d}\left(h, P_{\text {in }} ; \gamma_{\|\cdot\|}\right)=\frac{P_{\text {in }}}{\gamma_{1}} e^{-\gamma_{2} h}, \forall h \in \mathcal{H} \backslash h_{1} \\
g_{\angle \cdot, d}\left(h ; \gamma_{\llcorner.}\right)=\gamma_{3}+\gamma_{4} h, \forall h \in \mathcal{H}
\end{array}
$$

The load magnitude is be modeled as a decaying exponential function of the harmonic number. The phase angles are modeled as a straight line (as a function of harmonic number $h$ ) by "unwrapping" the phase angles. Defining device model parameter vector (for device $d$ ), $\gamma_{d}=\left[\gamma_{\|\cdot\|} \gamma_{L \cdot}\right]$,

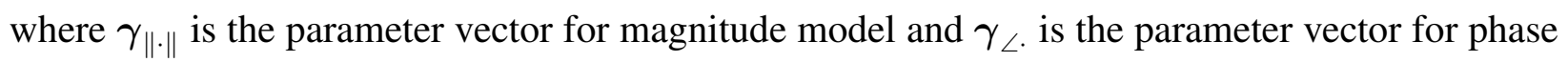
angle model, respectively. Function $g_{4}$, and the underlying equations is explained in detail in Appendix A. 


\subsection{Solving the Network}

The equations in (5.8) are complex valued. To solve the system using an iterative solver, the equations are rewritten in polar form and split into real and imaginary parts. The resulting state variables for the system of equations can be written in the form of a state vector as:

$$
\mathbf{x}=\left[\begin{array}{lllllllll}
\left\|\widetilde{I}_{1,1, h}\right\| & \angle \widetilde{I}_{1,1, h} & \left\|\widetilde{I}_{3,1, h}\right\| & \angle \widetilde{I}_{3,1, h} & \left\|\widetilde{I}_{4,1, h}\right\| & \angle \widetilde{I}_{4,1, h} & \left\|\widetilde{V}_{4,1, h}\right\| & \angle \widetilde{V}_{4,1, h} & P_{\mathrm{in}, 4,1}
\end{array}\right]
$$

Therefore, the total number of equations in the system are, $8 H+1$. These set of nonlinear equations can be solved using an iterative root finding algorithm. Newton Raphson (NR) is a popular algorithm that is widely used as it is easier to implement and the convergence is fast, given the problem is setup correctly with the right starting conditions.

The NR algorithm requires the computation of the system Jacobian. The Jacobian can be found using analytical techniques (symbolic math operations) or numerically. The example system was implemented and simulated in MATLAB. The MATLAB scripts and accompanying functions are listed in Appendix B.1. The system was simulated using the analytical and numerical Jacobian techniques, with both approaches resulting in convergence. The MATLAB script included in the appendix relies on numerical Jacobian.

The system was simulated for harmonics, $\mathcal{H}=\{1,3, \cdots, 19\}, h \in \mathcal{H}$. For the system, $H=10$ and the system contains only one nonlinear load, therefore, the resulting system Jacobian matrix is $81 \times 81$. System circuit parameters are as follows: $\widetilde{v}_{1}=120 \mathrm{~V}, \widetilde{v}_{h}=0 \mathrm{~V}$ for $h \neq 1, P_{\text {out }, 4,2}=1$ $\mathrm{kW}, \gamma_{1}=71.1, \gamma_{2}=0.2627 \times 10^{-2}, \gamma_{3}=-0.4261, \gamma_{4}=-0.18722, Z_{1}(h)=0.8+j 0.008 h \Omega$ and $Z_{2}(h)=25+j 0.301 h \Omega, \forall h \in \mathcal{H}$. Convergence parameter $\epsilon$ for the NR update equation was set to $1 \times 10^{-3}$.

For a successful convergence in Newton Raphson technique, the start values of the unknown state variables must be close to the final solution. Current values in terminal load devices can be obtained from the simple power calculation. In a practical electrical distribution, the wiring losses 
are low and therefore the voltage drop can be neglected. Voltage values of such devices can therefore set by specifying a nominal voltage value of the input voltage source. In case of a multiphase system, the nominal value of the voltage must also specify the phase angle that corresponds to the phase. Series impedance devices does not need an initialization value and can be set to zero.

Start values for the unknown state variables are set as follows: $\left\|\widetilde{I}_{1,1, h}\right\|=0, \angle \widetilde{I}_{1,1, h}=0$, $\left\|\widetilde{I}_{3,1, h}\right\|=0, \angle \widetilde{I}_{3,1, h}=0,\left\|\widetilde{I}_{4,1,1}\right\|=P_{\text {out }, 4} / 120,\left\|\widetilde{I}_{4,1, h}\right\|=0$ for $h \neq 1, \angle \widetilde{V}_{4,1, h}=0,\left\|\widetilde{V}_{4,1,1}\right\|=$ 120, $\left\|\widetilde{V}_{4,1, h}\right\|=0$ for $h \neq 1, \angle \widetilde{V}_{4,1, h}=0$, and $P_{\text {in }, 4,1,1}=P_{\text {out }, 4,2}$

The system converged to the final solution in 23 iterations. Final converged values for the simulated magnitude and (unwrapped) phase angles of the current through port 1, device 4 (nonlinear load) of the example circuit are shown in Figure 5.3.
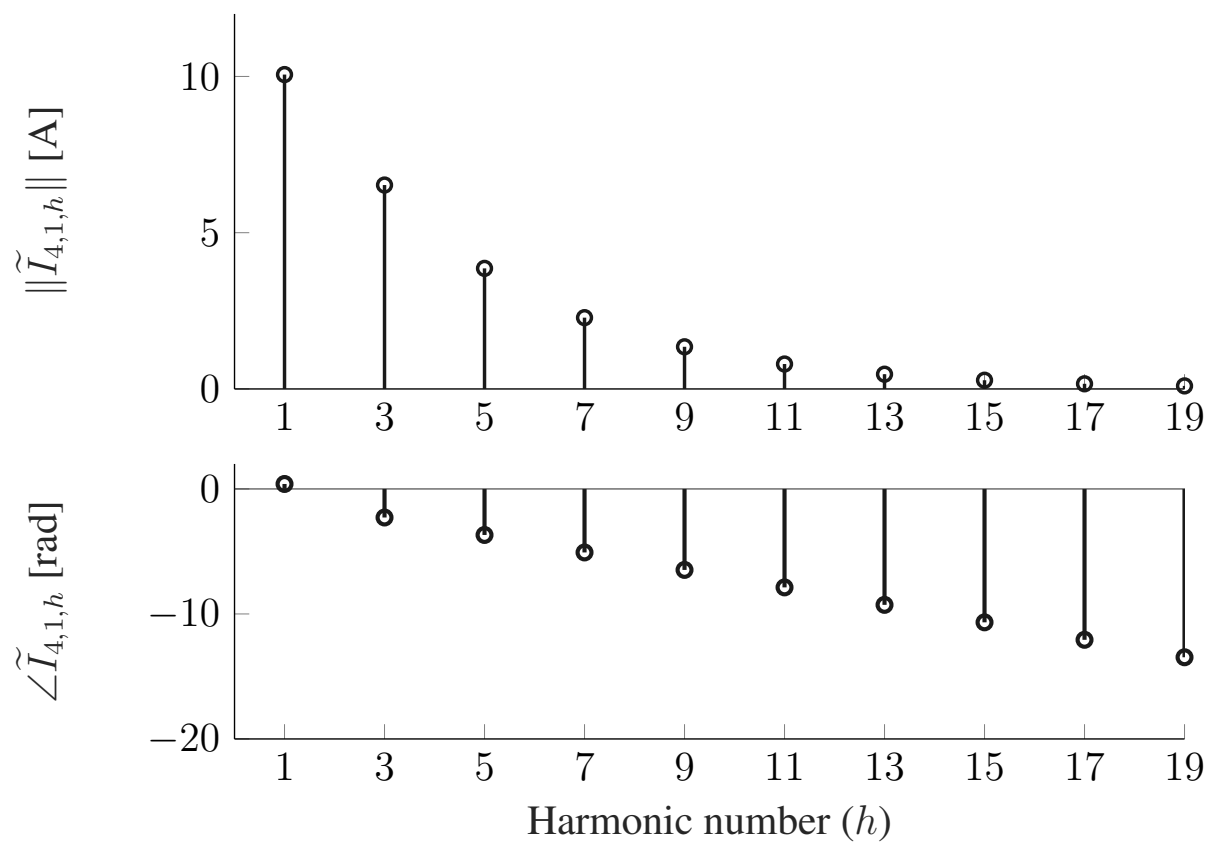

Figure 5.3: Simulated current magnitude at harmonics through branch 1 (top) and current phase angle at harmonics (bottom).

As can be seen in Figure 5.3, there is an exponential decay in the magnitude of the input port (device $d=4$ ) current and increase in the phase angle as the harmonic number increases, as expected from the device specific behavior model defined in (5.9). 


\section{Chapter 6}

\section{Software Implementation: Modeling Toolkit}

The harmonic power flow modeling framework described in the Chapter 3 gave an insight into the underlying mathematical framework of a harmonic power flow analysis. The preceding chapter describes an example system that was implemented in MATLAB and showed application of harmonic power flow analysis, solved using the Newton-Raphson technique. The numerical example requires user to manually setup the system equations and implement the solver. Such an implementation is not generic and therefore, not practically scalable to larger systems. Therefore, the requirement is to have a generic harmonic power flow analysis tool that can be easily scaled to larger systems and should be able to perform harmonic power flow analysis on multiple phase unbalanced systems. This chapter describes a harmonic power flow analysis framework implemented in a systems modeling language called Modelica.

\subsection{Modelica: A Systems Modeling Language}

Modelica is an object-oriented, equation based language for modeling and simulation of systems. Modelica was chosen for this research because it is an open source language and provides the ability to create functional mock-up units (FMUs) [51] [52], both of which enhance the speed and ease of integration with existing software toolkits (developed under previous Department of Energy funded efforts). Component models for physical systems are defined using the Modelica language. A complex interconnected system can then be built by describing relationships between models and therefore allows the user to build a complex system with various interacting components. The focus of the language is on modeling the system, and describing the behavior and interaction of underlying component models. The task of actual system simulation and solving of the differential and algebraic equations is left to the Modelica compiler. The language itself is freely available and standardized by the Modelica Association [53] and therefore, Modelica 'libraries'(collection of models and components written in Modelica) can be run on any Modelica tool [54]. 
Basic building block in Modelica is the model class. Model behavior is defined in the equation section that describes the relation between mathematical entities. Unlike imperative programming languages, equality operator $=$ is not an assignment operator. The equality operator has similar meaning as in a mathematical expression. This greatly simplifies the modeling of a system as the user is dissociated from the underlying solver implementation and can focus on system behavior description. This approach has immense implications, especially in power flow analysis. Assuming we have defined the voltage (v) and current (i) relation for a two pin model, the task of solving for voltage and current in a resistor $(\mathrm{v}=i \star r)$ is straightforward and can be done using a linear matrix inversion technique. For a power flow simulation, the equation section can be defined as $\mathrm{p}=\mathrm{V} \star \mathrm{i}$. The Modelica compiler can detect if the defined relation constitutes a linear or a nonlinear relationship and based on that, it can call the appropriate solver (e.g. linear matrix solver or a nonlinear iterative numerical solver).

The library has several advantages over any previous implementations of harmonic power flow. Conventional power flow analysis techniques, and its derivatives, such as the harmonic power flow use the admittance matrix approach to construct a system representation [30]. System interconnections are devised using a node indexing scheme and an admittance matrix is constructed. These power flow techniques are based on a nonlinear iterative solver such as the Newton-Raphson solver (NR) algorithm. The power flow formulations exploit the structure of the admittance matrix and the NR technique to come up with a set of equations that can then be used to solve for the system. There are several downsides to this approach, such as, difficulty in scaling to larger systems, integrating new device models require significant user effort, lack of standardized notation and use of different programming languages result in vastly different implementations that lead to difficulties in knowledge exchange.

Modelica lends itself to be highly useful for modeling and simulation of power flow problems. A power flow formulation consists of various interconnected devices, such as voltage sources, line impedances, and loads. In Modelica, the device behavior is defined using mathematical relations. System interconnects are defined using specialized connector class. This approach puts the focus 
of the user on actual system representation and modeling system behavior. The actual task of generating system representation for the purpose of computations is left to the Modelica compiler. The advantages offered by an equation based language in modeling a power flow formulation is reflected in the fact that an example power flow formulation was implemented in the original equation based modeling language [55]. The proposed language was a precursor to the modern Modelica language.

It is worthwhile to mention other Modelica libraries for Modeling electrical distribution systems. Modelica Standard Library includes a phasor based steady state simulation (at fundamental frequency) library called Modelica Electrical QuasiStationary library [56]. The library can simulate stead state systems using phasors but does not have device models for a power flow analysis. The Modelica PowerSystems Library [57] is a full-fledged library for fundamental power flow analysis. The library contains a wide variety of device models for modeling a power system. Modelica Buildings Library [58] has a rich suite component models for electrical distribution system simulation.

\subsection{Component Library}

The toolkit library is defined in a Modelica package class named HPF. All components are referenced using this package name. Components in the toolkit are organized by groups and their logical interconnections. This grouping specifies an equipment hierarchy which is used to internally reference components and their interconnections and to aid users in searching and displaying available components. The hierarchy includes:

- Sources: single and three-phase AC voltage sources, DC voltage sources.

- Sensors: measurement sensors for voltage, current, power, and data probes.

- Transformers: single and three-phase AC transformers.

- PowerConverters: power electronic converter models such as AC to DC rectifiers, power supplies, and other MELs. 
- Loads: various loads, such as electrical motors, heaters and generic AC and DC loads.

Figure 6.1 gives an overview of the library.

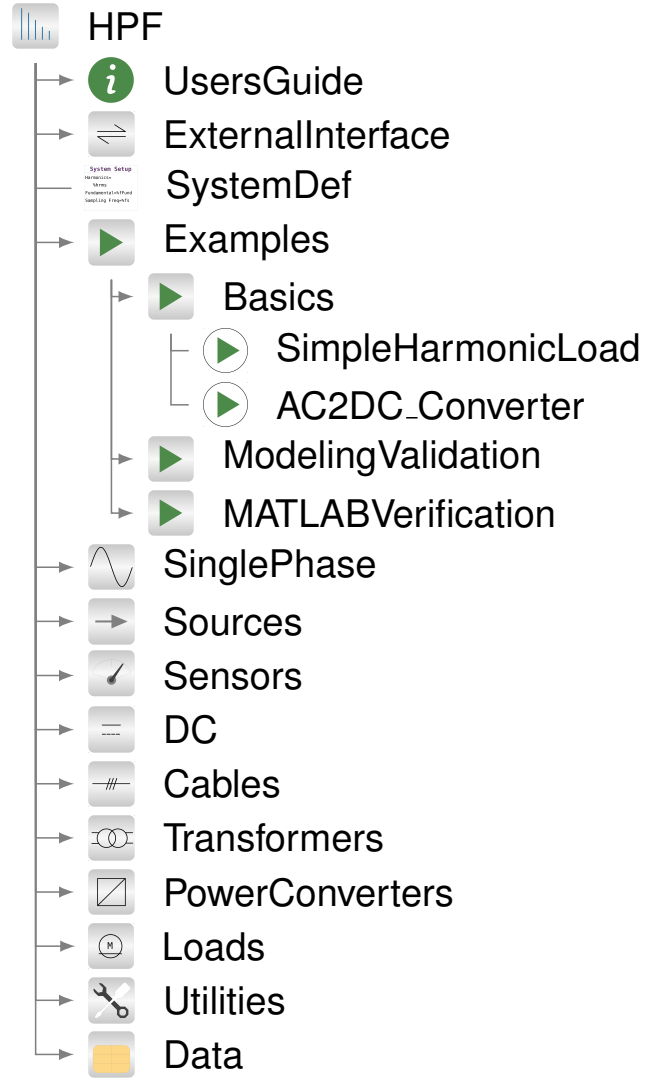

Figure 6.1: Partial expanded view of Modelica toolkit library as seen in a Modelica environment, showing various library component models and constituent packages.

Basic circuit elements for constructing a single phase distribution can be found in SinglePhase package. Examples package includes examples on a basic harmonic power flow implementation. Data package contains data files and Modelica records for device models.

Components associated with nonlinear devices which contribute harmonic content in the system, such as power electronic converters, are modeled according to their nonlinear voltage vs. current harmonic relationship, as described in chapter 3. Purely AC and DC loads are modeled as lumped devices. Users manually set the parameters and/or power usage of the device as fixed input parameters. Also included in the toolkit library are simplified DC/DC converter models which 
are based on device efficiency curves. These converter models compute an output power estimate based on input power as described in [59].

\subsubsection{Component Connections}

As a model-based simulation language, variables in Modelica represent data flow within the modeled system. In a conventional fundamental power flow simulation, complex variables are used for current and voltage (frequency is constant). In the toolkit developed in this work, frequency harmonics in the system are represented using vectorized variables, where elements of these vectors contain the ordered voltage current harmonics.

The Modelica language specification defines a special class for defining terminals called 'connector'. The toolkit utilizes this class to define a connector class referred to as a 'harmonic pin' as follows:

1 connector hPin

2 parameter Integer $h=1$;

3 Complex $v[h]$;

4 flow Complex i[h];

5 end hPin;

In the code above, the connector is defined with variable hPin. The connector class variables are a vector of Complex type; voltage is represented by complex variable array $\mathrm{v}$ of size $\mathrm{h}$; similarly, flow variable $i$ represents current. The size of the harmonic connector is set by the parameter $h$, that is passed to the underlying voltage and current variables. When utilized in a component, the connector class object defined above simplifies the manipulation of complex voltage and current harmonics at all nodes and branches of the system.

The parameter $\mathrm{h}$ is a systemwide variable that sets the maximum number of harmonics $H$ in the simulation. A typical simulation can have any number of device models built using the harmonic pin connector, therefore the connector parameter $\mathrm{h}$ is defined on a system wide level using a global class SystemDef, shown in the Figure 6.1. The parameter is inferred from another global variable defined in the SystemDef class, called hrms. The hrms variable sets the harmonics to be 
simulated in a system. For example, in a system with symmetric steady state waveforms, one can omit the even harmonics. In such a system, the harmonics vector (e.g. the first 5 odd harmonics) will be defined as, hrms $=\{1,3,5,7,9\}$.

The flexibility provided by the global variables has huge implications on a simulation model in harmonic analysis. The size of the harmonics vector determines the level of accuracy of a harmonics simulation. A simulation with a large number of harmonics can result in a system that is substantially large, resulting in convergence issues. Therefore, by setting the harmonics vector, user can strike a balance between accuracy and system stability in regard to computational resources.

\subsubsection{Component Models}

The partial class twoP inBase defines a base class that establishes relations between the two pins of a two terminal device. To model an electrical element, the partial class is 'extended' and the variables defined in the partial class are then used to model the device characteristics as:

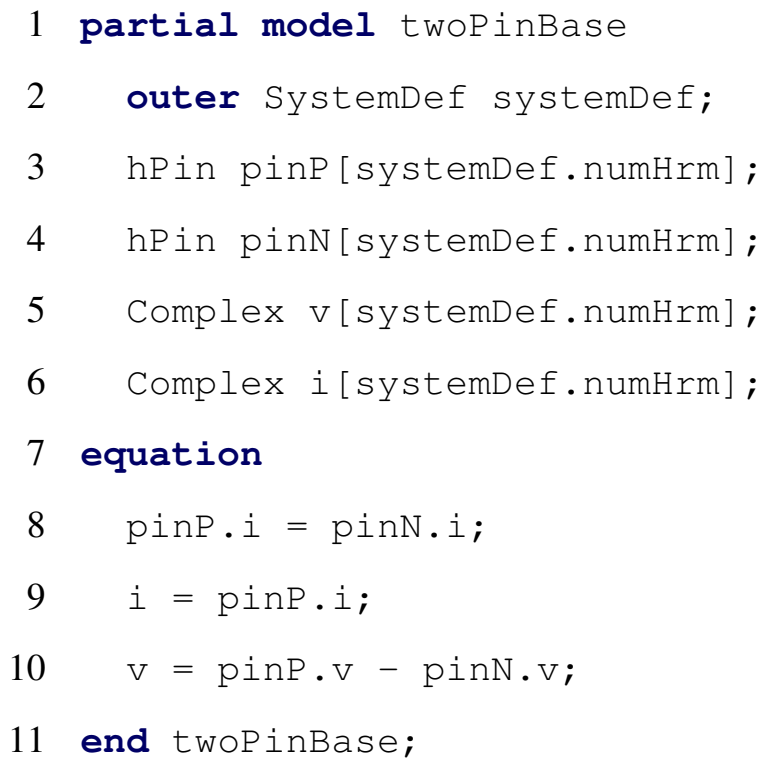

In the code above, the first line creates the partial class twoP inBase. The second line creates an object of the SystemDef class that defines system wide parameters. The terminal pins are created in the outer section. The equation section defines the relation between variables of the two pins, which also enforces conservation of flow (currents) throughout the model. 
Circuit elements are then created as follows. First, a complex 'base' impedance $Z$ is specified at the fundamental frequency (i.e., associated with harmonic $h=1$ ). Impedance at harmonic $h$ is then computed as $Z(h)=\operatorname{Re}\{z\}+j h \operatorname{Im}\{z\}$. An example for a resistive-inductive element is shown below:

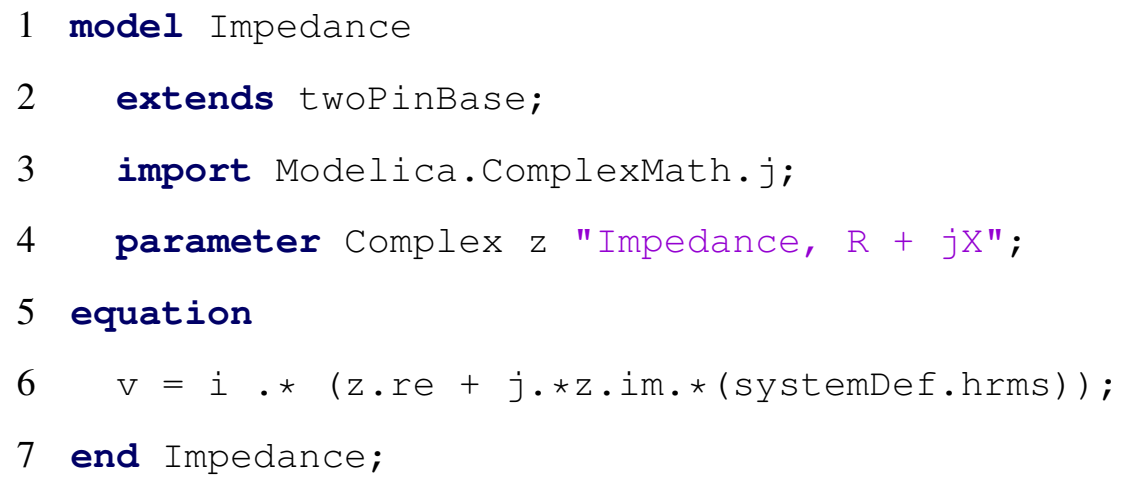

In the code above, the model inherits the vector of hamonics systemDef.hrms defined in the systemDef block; $j$ is the imaginary unit (imported from Modelica Std Library, defined as, final constant Complex $j=\operatorname{Complex}(0,1))$, . re and . im are the real and imaginary operations, respectively, defined in the Modelica Standard Library (Modelica.ComplexMath.j).

Associated with all components is a graphical two-terminal icon, illustrated for a simple impedance element in Fig. 6.2.

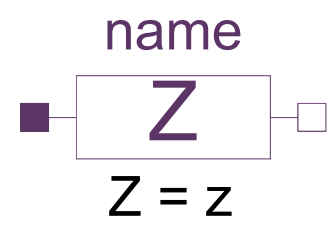

Figure 6.2: Graphics layer showing internals of $\mathrm{AC} / \mathrm{DC}$ converter.

In Fig. 6.2, name refers to the annotation (name of the object of class Impedance) for the model when included in a simulation. Values for the base impedance are set by the variable $z$ by clicking on the icon (in a graphical tool), or by passing value to the parameter (for an object of 
class Impedance) in the Modelica code. Similarly, all the basic circuit elements can be constructed, namely, Resistor and Capacitor.

Converters are modeled as two port devices. An example of a two-port device model created in the toolkit, Figure 6.3 shows the graphics layer of an AC/DC converter, where left side of the component is the "input" port, consisting of $\mathrm{AC}$ harmonic voltage and currents. The input port is modeled in the form of an extended loadBase class, which itself is extended from the twoP inBa se one-port base class described above. The right side of the component in Figure 6.3 is the "output" port, representing the DC side of the converter. The DC side is a controlled DC voltage source, which can be connected to other DC devices in the network.

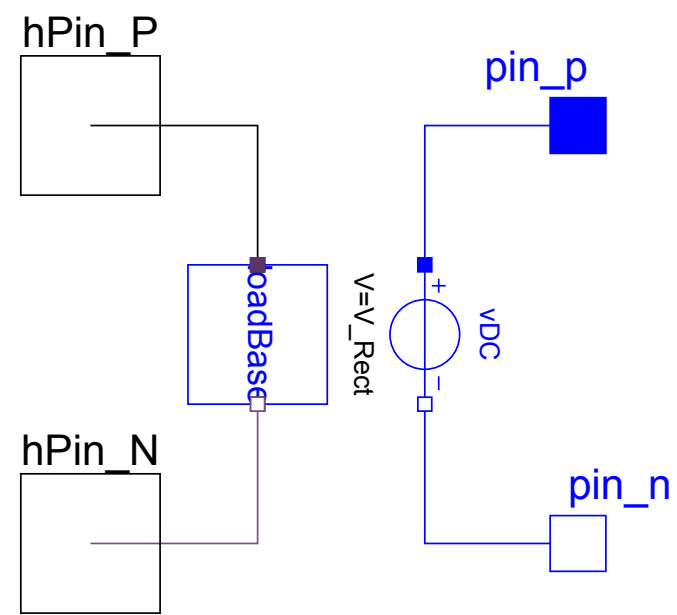

Figure 6.3: Toolkit icon for an impedance element.

The Modelica code below shows a partial listing of the underlying component objects in the AC/DC converter model. Relations defining the harmonic power flow, corresponding to the device model described in section 4.4 are defined in the equation section. (See toolkit source code for the complete Modelica code.)

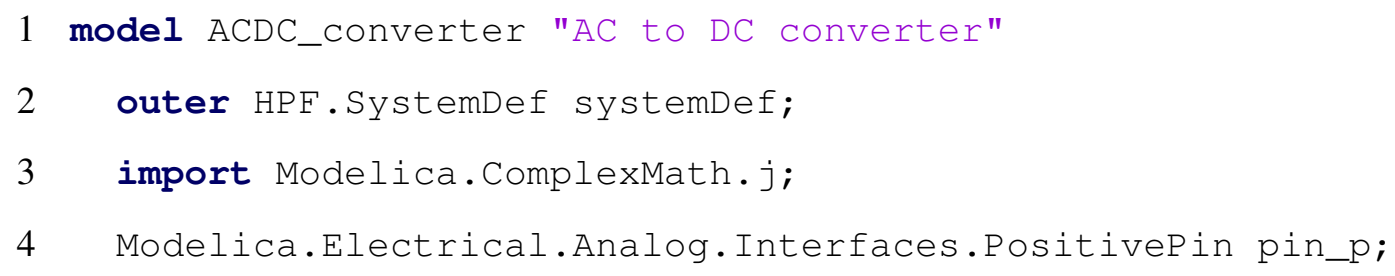




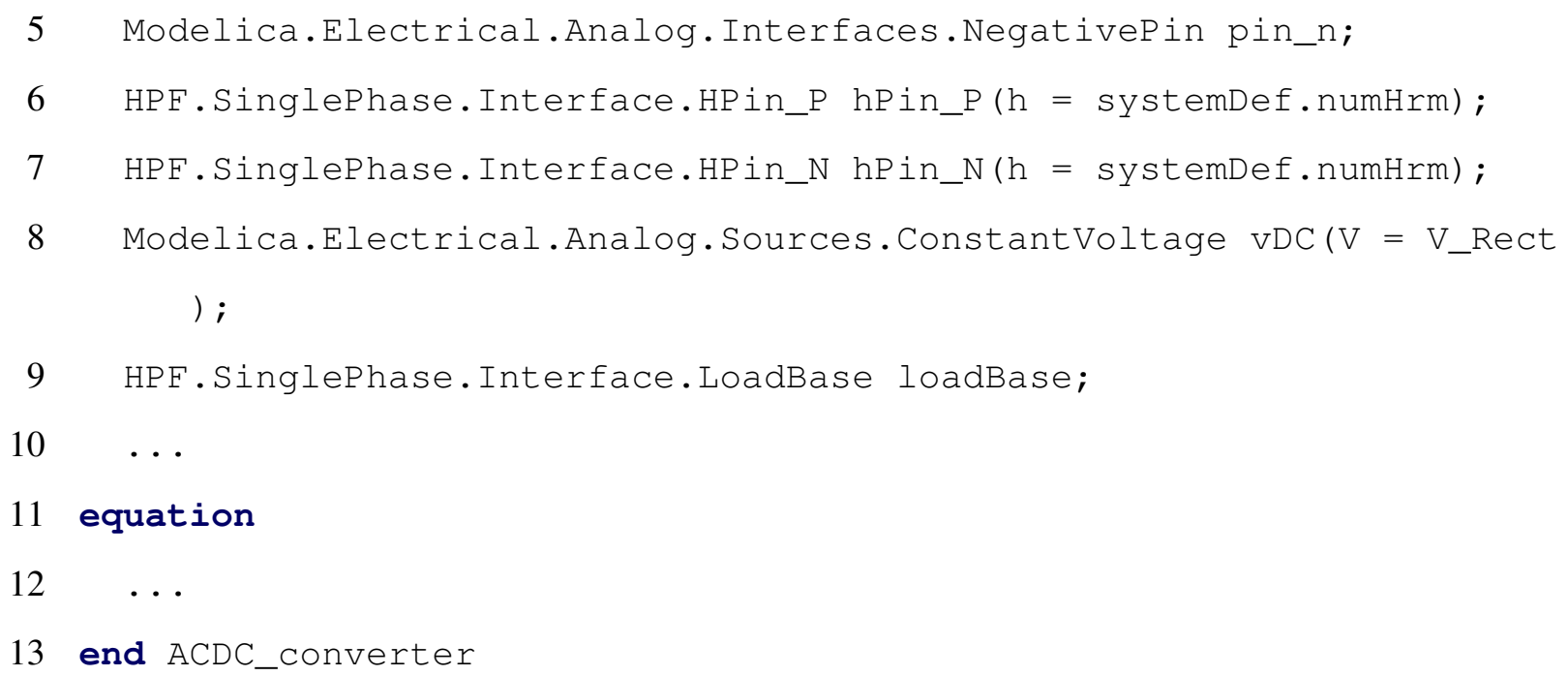

Benefits of an object-oriented modeling language is most evident in the modeling of the threephase transformer. The model illustrates model reuse concept that is central to the Modelica language. The three-phase transformer model begins with a single-phase ideal transformer that is used to build a harmonic T-model that models primary, secondary and core losses using basic harmonic circuit elements. Figure 6.4 shows the model of a three-phase delta-wye transformer (A) and the underlying models. (A) shows the graphics layer with the interfacing harmonic connector objects for the primary and the secondary side. The three-phase transformer model is built by creating three objects T1, T2 and T3 of the model class HPF.Transformers.SinglePhase. TModel shown in (B). Similarly, the model uses an object from ideal transformer model (shown in (C)).

\subsection{Initialization}

Harmonic power flow analysis is a nonlinear system and therefore requires an iterative numerical solver. For numerical solvers such as Newton-Raphson, convergence is guaranteed only if the solution is close to an initial starting point. Therefore, a fair amount of effort goes into ensuring proper initializations for the solver. Modelica has a provision to specify initial conditions for component models at the beginning of a simulation. Each variable in Modelica can be assigned a start attribute that is used to set the initial value. The start value, by default, is a suggested value for the solver. A solver, in the initialization phase, may choose to set a different start value. User 


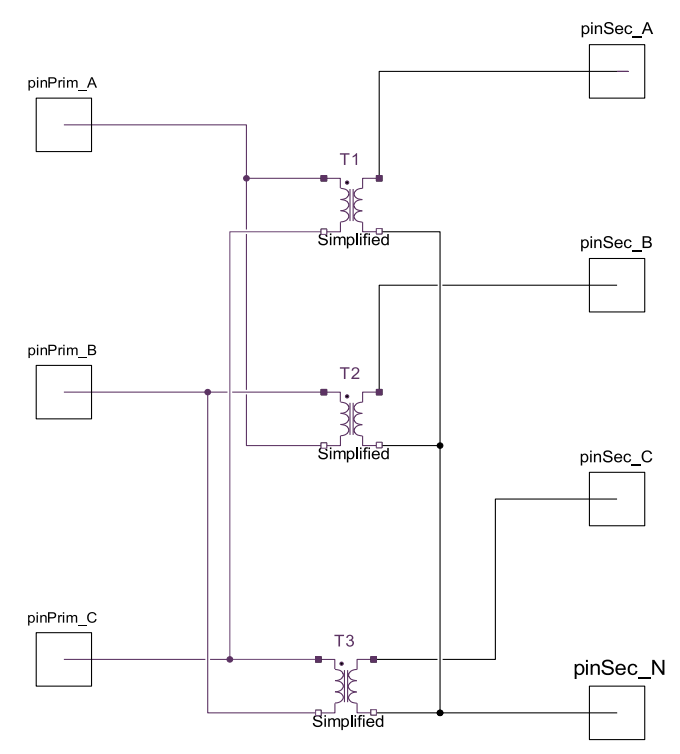

(A)

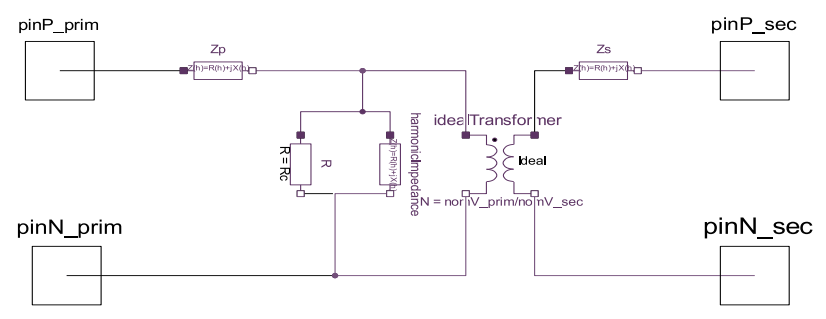

(B)

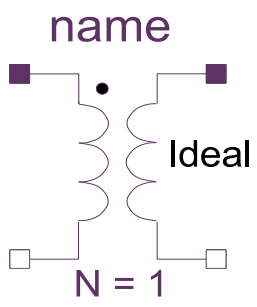

(C)

Figure 6.4: Three-phase transfomer model (A) from the library along with constituent models for T-model (B) and ideal transformer (C).

can override the solver suggest start value by setting the fixed attribute to true. As an example, real power at $h=1$ for a nonlinear load is defined as follows:

$$
\text { Real P(start }=1000 \text {, fixed = true) "Real power"; }
$$

Start values in models are set by setting 'Nominal' variable values. User can either choose to leave the nominal value unchanged or override the default options by manually specifying the start values. The values are accessible via the device model icon dialog box. All single port devices, such as a resistor, have an initialization tab to manually specify start values to the underlying voltage and current variables. This is useful in scenarios that encounter convergence issues not remedied by component nominal values.

\subsection{System Solver}

As a physical system modeling language, Modelica insulates user from the underlying solver implementation, allowing user to focus on modeling system behavior. Solving of system equations as described by the language is offloaded to the underlying Modelica compiler. 
Every variable in Modelica is time-stamped by default. For a linear system comprising of ordinary differential equations, Modelica solves the system using numerical integration. However, harmonic power flow is a nonlinear algebraic problem and therefore requires an iterative nonlinear solver. Given a defined nonlinear algebraic system, Modelica compiler chooses from among its installed iterative root-finding algorithms to solve the system. Newton-Raphson solver, a popular nonlinear solver, is installed by default in Dymola and OpenModelica.

In the work described here, the library was used to compute steady-state solutions for voltages and currents needed for estimation of steady state device and system efficiencies. However, because Modelica variables are time-stamped, the toolkit can be called sequentially to simulate quasi-static, time-varying loading conditions (i.e., a series of steady state solutions which neglect transient effects), such as hourly load variations in a building over a 24-hour time period.

\subsection{Solution for Example System}

To validate the Modelica implementation of the toolkit, in particular to verify harmonic injections arising from nonlinear circuit elements, solution of the example circuit described in chapter 5 was performed and is discussed in this section.

The graphical representation of the electrical network in Figure 5.1 using the toolkit is shown in Figure 6.5. System simulation parameters are defined in a top-level block called 'System Setup' which assigns variables within the SystemDef class. For the example simulation, the system parameters were set to: $\mathrm{hrms}=\{1,3, \ldots 25\}$ (harmonics to be simulated); fFund $=60 \mathrm{~Hz}$ (fundamental frequency); fSamp $=5 \times 10^{3} \mathrm{~Hz}$ (sampling frequency when visualizing time-domain waveforms). The nonlinear load device is modeled using the component block 'load'. Values for the base line impedance (Z01) and base load impedance (Z12) are are as displayed in the figure. Convergence parameters for the iterative solver was set to $1 \times 10^{-3}$. The equivalent Modelica code for the system shown in Figure 6.5 is listed in Appendix B.2.1. Initialization procedure for an iterative numerical solver requires proper start values for the system-wide unknown state variables. 
In the example system, start values for the nonlinear load and the impedance $\mathrm{z} 2$ are set by passing a vector to variable start_v_re.

\section{Load model in Modelica}

Modelica code for the load model load is listed in Appendix B.2.2. Lines 25-28 correspond to the device harmonic spectra model described in (5.8). Line numbers 19-21 correspond to the power balance over all harmonics, $(5.8 \mathrm{e})$.

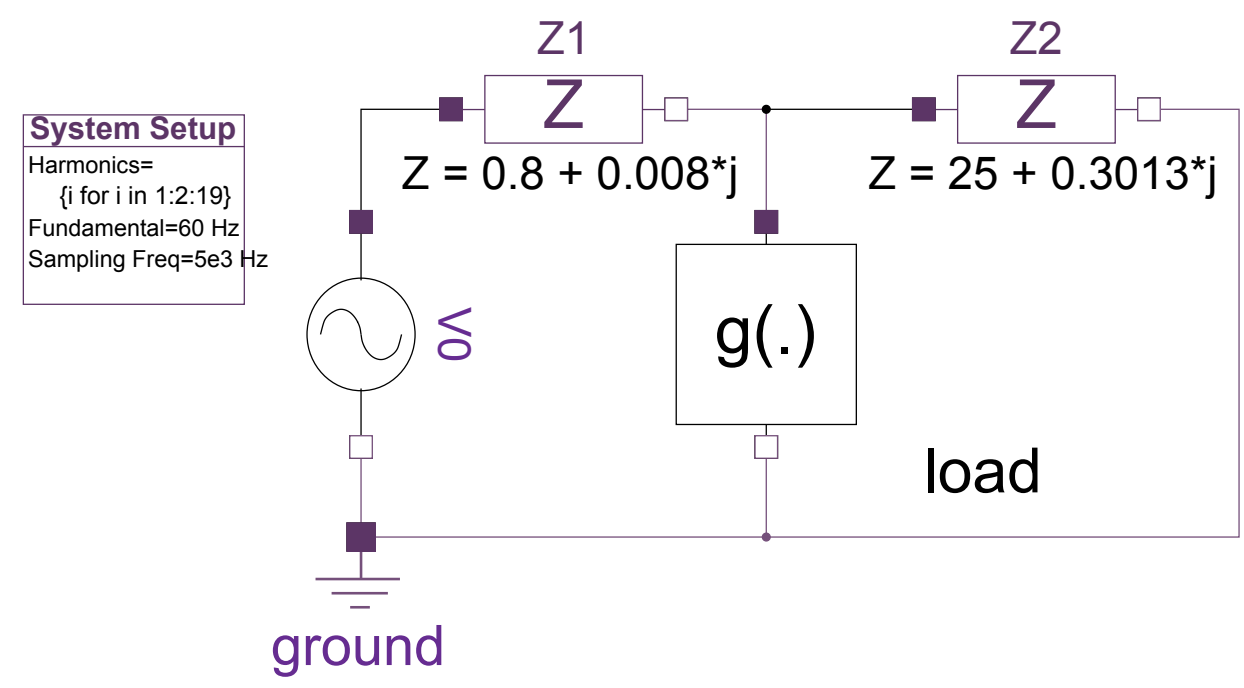

Figure 6.5: Graphical representation of example system within toolkit.

\section{Simulation results}

During the model 'flattening' procedure, the Modelica compiler generates a flattened model with equations for each harmonic. In this example, the flattened model generated 618 equations in both Dymola and OpenModelica. In Table 6.1 the toolkit output is compared to MATLAB. As shown in Table 6.1, the toolkit correctly computed the current harmonics and phase angles for the nonlinear system, to four decimal places, compared to the scripted MATLAB solution. Total simulation time for the toolkit to solve the system was $1.6 \mathrm{~ms}$; solution using scripted MATLAB required $104.0 \mathrm{~ms}$. The solver converged to the final solution in 20 iterations 
Table 6.1: Simulation output comparison on nonlinear example problem: Toolkit vs. Scripted MATLAB.

\begin{tabular}{c|c|c|c|c}
\hline & \multicolumn{2}{|c|}{ Toolkit } & \multicolumn{2}{c}{ MATLAB } \\
\hline$h$ & $\left\|\widetilde{I}_{4,1, h}\right\|$ & $\angle \widetilde{I}_{4,1, h}$ & $\left\|\widetilde{I}_{4,1, h}\right\|$ & $\angle \widetilde{I}_{4,1, h}$ \\
\hline 1 & 10.6526 & -0.3973 & 10.6526 & -0.3973 \\
3 & 6.7418 & 2.8922 & 6.7418 & 2.8922 \\
5 & 3.9866 & 0.7564 & 3.9866 & 0.7564 \\
7 & 2.3573 & -1.3795 & 2.3573 & -1.3795 \\
9 & 1.3939 & 2.7679 & 1.3939 & 2.7679 \\
11 & 0.8243 & 0.6320 & 0.8243 & 0.6320 \\
13 & 0.4874 & -1.5038 & 0.4874 & -1.5038 \\
15 & 0.2882 & 2.6435 & 0.2882 & 2.6435 \\
17 & 0.1704 & 0.5077 & 0.1704 & 0.5077 \\
19 & 0.1008 & -1.6281 & 0.1008 & -1.6281 \\
\hline
\end{tabular}




\section{Chapter 7}

\section{Nonlinear Device Behavior Modeling}

In chapter 4.4.1 (chapter 4), device-specific behavioral functions (DBFs) were approximated using linear interpolation based on device measurements; the interpolated values were computed iteratively at each step in the HPF solver routine. However, it is desirable to obtain closed-form parameterized DBFs for common power-electronic devices, to obtain faster evaluation of the functions and their derivatives, the latter of which are used in common HPF solvers that iteratively compute numerical matrix Jacobians of the system equations. This chapter describes a procedure for determining closed-form parameterized device-specific behavioral functions described by nonlinear AC/DC rectifiers. These models are intended for use in HPF-based numerical modeling packages such as BEEAM.

\subsubsection{AC/DC Converter Harmonics}

In the simplest form, an $\mathrm{AC} / \mathrm{DC}$ converter consists of a single phase line-commutated diode bridge rectifier and a filter capacitor that smooths the voltage ripple. Some rectifiers include additional voltage regulation electronics, often in the form of a DC/DC conversion stage. A notional circuit is depicted in Figure 7.1 .

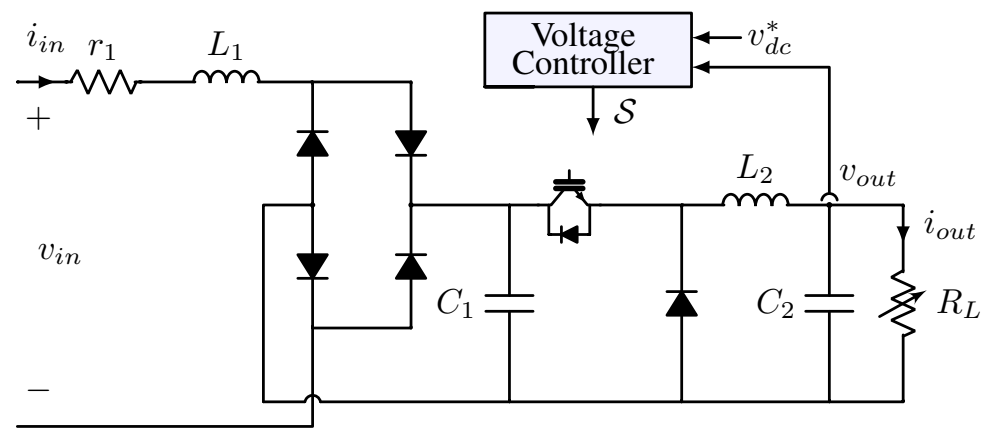

Figure 7.1: Notional rectifier with output DC voltage regulation. 
In Figure 7.1, $v_{\text {in }}$ and $i_{\text {in }}$ are the input AC voltage and current respectively; $v_{\text {out }}$ and $i_{\text {out }}$ are the output DC voltage and current, respectively; $r_{1}$ and $L_{1}$ are input resistance and inductance, respectively; $C_{1}$ and $C_{2}$ are filter capacitors; $L_{2}$ is a filter inductor for the DC/DC converter; $R_{L}$ is the output load resistance. Feedback control is depicted by the "Voltage Controller" block, that does voltage regulation by measuring output voltage and setting the DC/DC converter duty cycle.

The filter capacitor $C_{1}$ in Figure 7.1 provides a degree of initial voltage ripple smoothing on the output of the diode bridge. As a consequence of $C_{1}$, the diodes in the bridge conduct only when the voltage across $C_{1}$ is below forward voltage of the diodes. This results in the bridge conducting for a limited duration over half of an electrical cycle, which results in significant current waveform distortion as shown previously in Figure 2.2.

In HPF formulations, nonlinear devices, such as rectifiers, are modeled as current source injections at odd harmonic frequencies. One technique for introducing current injections into a simulated electrical networks is using fixed injection spectra. This modeling technique relies on an AC current measurement of the nonlinear device under a fixed (e.g., rated) load. The measured injection spectra from this measurement are then used to model the nonlinear device as current sources with amplitude and phase angles determined by the spectra obtained by the single measurement. However, a drawback to this approach is that the injection spectra are a function of the device input power [3]. Figure 7.2 illustrates the relationship between rectifier input current (top) and the magnitude of the current harmonics (bottom) on the output power for an experimental rectifier.

The figure shows that increasing power levels result in not only higher peak currents, but also a widening of waveform and shift in the location of waveform peaks. This indicates that harmonic spectra obtained from measurements at a single operating point cannot be scaled linearly and the harmonic content of the waveforms exhibit a nonlinear relationship with device power.

Several other factors determine the harmonic spectra of current drawn by rectifiers, which can vary depending on the rectifier design and modes of operation. For instance, the values of the fixed parameters in Figure 7.1, the type and nature of additional voltage conditioning and/or power factor correction circuits (if they exist) and their control strategy, etc., may impact the shape of the 


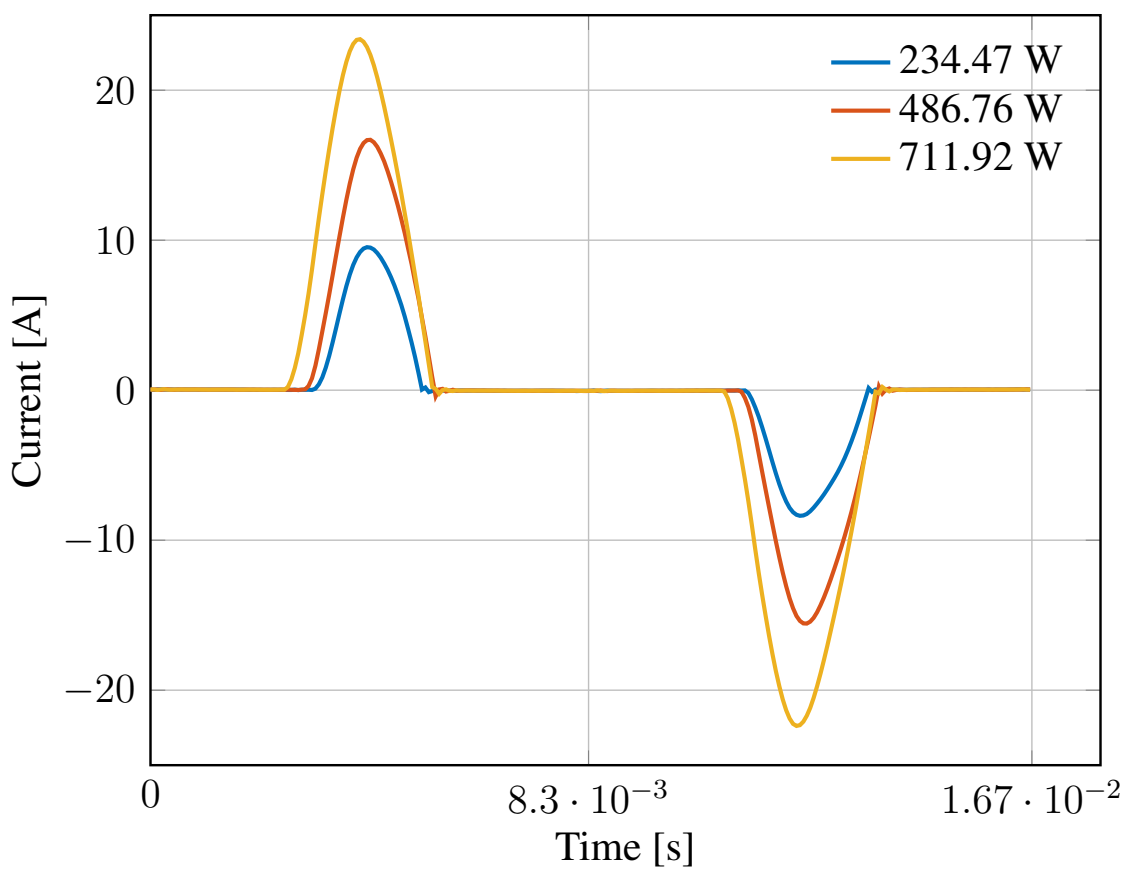

(a) Time domain current waveform vs. power level.

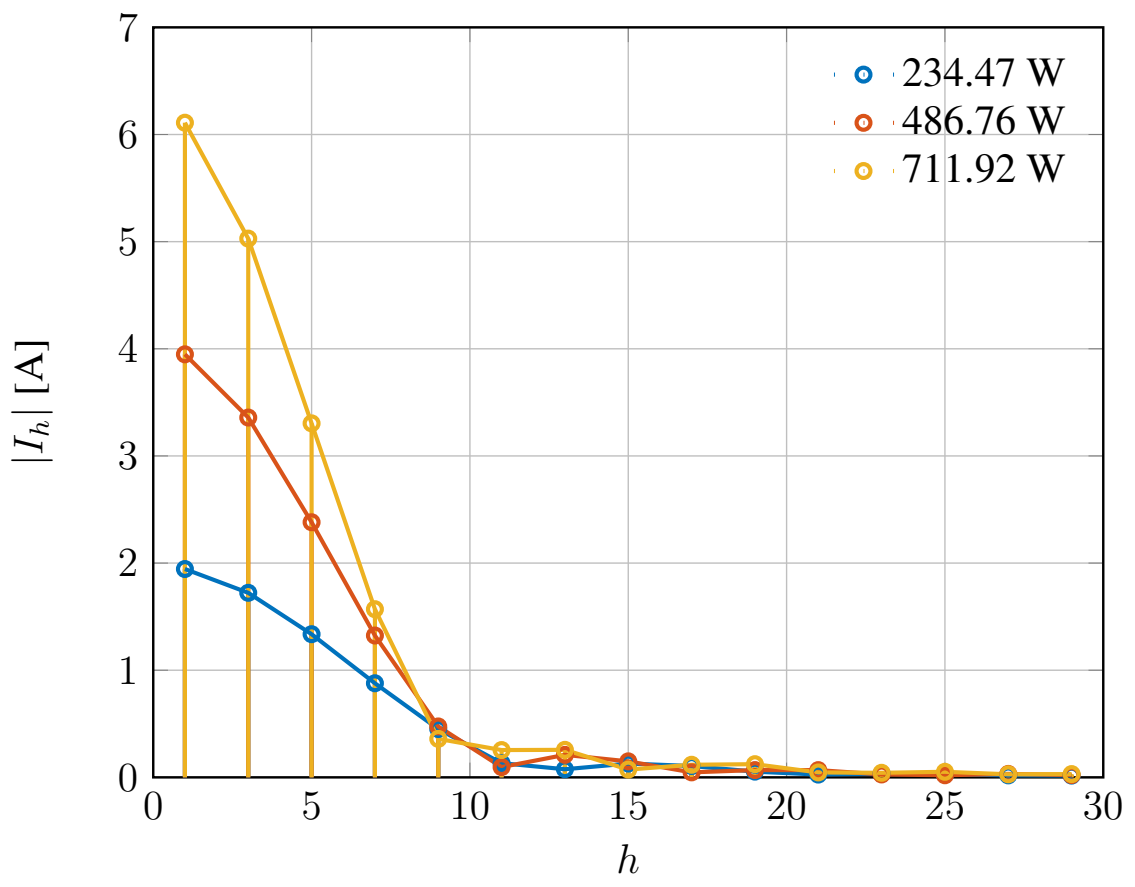

(b) Magnitude of current harmonics vs. power level.

Figure 7.2: Rectifier input current waveform (a) and equivalent harmonic magnitudes (b) vs. power level.

current waveform. In practice therefore, commercial rectifiers can exhibit vastly different harmonic spectra. 
The empirical harmonic model for the AC/DC converter presented in chapter 4 is a refinement over the fixed injection spectra method as it approximates the nonlinear behavior of rectifiers using measured harmonic spectra at varying power levels. The empirical models the converter harmonics by assuming locally linear behavior about an operating point (determined by the harmonic number and converter operating power), and estimating the current harmonic and phase angles by interpolation between their nearest measured values.

Figures 4.5 and 4.6 show that the current harmonics exhibit a nonlinear relationship with the harmonic number and the input power; however this behavior displays a reasonably "wellbehaved" and smooth contour when plotted with respect to these two dimensions.

Despite the improvements in accuracy using interpolation versus fixed harmonic spectra approach, this method also presents several drawbacks that can be improved by developing closedform parameterized DBFs to represent nonlinear device behavior. First, performing interpolation is less computationally efficient than closed-form function evaluations. Second, network equation solvers often make use of matrix Jacobians by evaluating numerical derivatives; it is advantageous to develop DBFs with closed-form, smooth first derivatives. Third, using the interpolation approach is limited to the specific devices that can be measured; it is desirable to obtain parameterized DBF models that are representative of a range of devices. The next section describes the proposed model.

\subsection{Device-specific Behavioral Function Models}

This section describes the proposed device specific behavioral function models. Behavioral model parameters are contained in the parameter vector defined as:

$$
\gamma=\left[\gamma_{1}, \gamma_{2}, \ldots\right]
$$




\subsubsection{Harmonic Magnitude Model}

The converter AC side current harmonic magnitudes are modeled using a logistic function. The logistic function belongs to the sigmoid function family. Some of the desired characteristics of the function are, monotonically decrease to zero, as, $\lim _{x \rightarrow \infty} f(x)=0$. This characteristic is desired because most of the harmonic content is present in the lower five or six odd harmonics and the magnitudes eventually decay to zero. Other desirable characteristics are, a sigmoid type decay, the ability to set the max value (this corresponds to the fundamental harmonic), and the ability to set the rate of decay. A generic sigmoid function has the following form,

$$
f(x)=\frac{a}{b-e^{-c x}}
$$

The sigmoid function in one variable can be extended, from a two-dimensional function in one variable to a three dimensional function in two variables, by parameterizing the function along $y$ axis. An important feature that a candidate model must capture is the sigmoid type decay in the harmonic magnitude as a function of the converter power. The decay is controlled by parameter $c$ in (7.2). This feature is modeled by replacing $c$ with an exponential function that is the function of converter power. Proposed model for the converter device specific behavior is given as:

$$
\left\|g_{d}\left(h, P_{d, 1} ; \gamma\right)\right\|=\frac{\gamma_{1} P_{d, 1}+\gamma_{2}}{\frac{\gamma_{1} P_{d, 1}+\gamma_{2}}{\gamma_{3} P_{d, 1}+\gamma_{4}}-e^{-\left(\gamma_{5} e^{-\gamma_{6} P_{d, 1}}\right) h}}
$$

\subsubsection{Harmonic Angle Model}

Current harmonic angles are modeled using a polynomial function in two variables. The function represents a curved surface in three dimensions. For the purpose of harmonic angle modeling, the angles from measured data are "unwrapped" (not restricted to $[0,2 \pi]$ or $[-\pi, \pi]$ ). The model is stated as: 


$$
\angle g_{d}\left(h, P_{d, 1} ; \boldsymbol{\gamma}\right)=\gamma_{7}+\gamma_{8} h+\gamma_{9} P_{d, 1}+\gamma_{10} h P_{d, 1}+\gamma_{11} P_{d, 1}^{2}
$$

\section{Weights}

Converter harmonics phase angles modeled using the surface polynomial function have some limitations. A rectifier operating at low loads results in small current draw on the AC side. The result is that the current waveform peaks drop resulting in a near sinusoidal waveform with no distortion. Harmonic components at higher harmonic numbers become difficult to resolve using an FFT algorithm. As consequence, the computed angles can exhibit wide variations. This is also true for angles at higher harmonic number across all power levels. A fitting routine using a polynomial function cannot capture these angles and is therefore bound to result in erroneous fits.

The fitting routine utilizes weights [60] that selectively ignore phase angles from measured data at higher power levels and higher harmonic numbers. The weights also ensures that angle for the fundamental frequency $(h=1)$ is captured at all power levels. (Note: The weights are not used for the harmonic magnitude model.)

\subsection{Parameter Estimation}

This section describes the objective function for the parameter estimation and the optimization technique. Notation $\widehat{\{\cdot\}}$ refers to estimated value using the proposed models. The variable notation described in chapter 3 is modified to accommodate current harmonics for various converter operating power levels. Therefore, the new notation is defined as, $\widetilde{I}_{d, 1, h, P}, \forall P \in \mathcal{P}_{L}$, where $\mathcal{P}_{L}$ is the set of input real power at $h=1$. Restating for consistency, $\mathcal{H}=\left\{h_{0}, h_{1}, h_{2}, \ldots, h_{H}\right\}, h_{i} \in \mathbb{W}$ is a set of harmonic numbers. Supplementary functions for parameter estimation are defining as:

$$
\begin{aligned}
& q_{1, h, P}(\gamma)=\left\|\widetilde{I}_{d, 1, h, P}\right\|-\left\|\widehat{\widetilde{I}}_{d, 1, h, P}\right\| \\
& q_{2, h, P}(\gamma)=\angle \widetilde{I}_{d, 1, h, P}-\angle \widetilde{\widetilde{I}}_{d, 1, h, P}
\end{aligned}
$$


The measured data is in the form of harmonic spectrum at odd harmonics for a total of $H$ harmonics taken at total of $P_{L}$ power levels. The weights are defined as, $w_{i, h, P}$, where $i$ is the index for supplementary function, $h$ is the harmonic number and $P$ is the converter operating power. The weights are ignored for the harmonic magnitude model, therefore, $w_{1, h, P}=1$. The optimization problem is the classical curve fit problem that minimizes the root mean square value of the supplementary functions in (7.5). The objective function for parameter identification is stated as:

$$
\gamma^{*}=\arg \min _{\gamma}\left\{\sqrt{\frac{\sum_{i=1}^{2} \sum_{h \in \mathcal{H}} \sum_{P \in \mathcal{P}_{L}} w_{i, h, P}\left(q_{i, h, P}(\gamma)\right)^{2}}{2 H P_{L}}}\right\}
$$

The optimization problem stated in 7.6, is solved using particle swarm optimization (PSO). PSO solves a problem by moving a population of candidate solutions in the search-space. The movement of the particles is governed by a mathematical formula for the particles velocity and position. PSO is a metaheuristic algorithm that lends itself to be highly effective in optimization problems involving high dimensionality and nonlinear objective functions [61].

PSO algorithm parameters are as follows: the search space for parameters was set at $[-5,5]$; swarm size was set at 1000 particles; particle inertia was set at 0.7 ; velocity scaling term was set at 1.3; maximum iterations (termination criteria) was set at 8000 . The optimization routine was run for nine devices. Most devices reached a best fit solution in under 5000 iterations with no significant change in fitness above 7000 iterations.

Table 7.1 lists the rms error from the characterization procedure for DBF harmonic magnitude and angle models. The devices under test are described in chapter 8.

Figure 7.3a shows the fitted harmonic magnitude model (surface plot) superimposed on the measurement data (black markers). The model is able to capture all the important features from the measurement data. Figure 7.3b shows the fitted harmonic angle model (surface plot) superimposed on the measurement data (black markers). Notice the surface plot shows deviation from the 
Table 7.1: Devices characterized in the experiment.

\begin{tabular}{lcc}
\hline Device alias & RMS error, $q_{1}$ & RMS error, $q_{2}$ \\
\hline LED Driver 1 & 0.041 & 0.0132 \\
LED Driver 2 & 0.036 & 0.0129 \\
LED Driver 3 & 0.028 & 0.0147 \\
Laptop Charger 3 & 0.161 & 0.0019 \\
Laptop Charger 4 & 0.098 & 0.0038 \\
Laptop Charger 5 & 0.122 & 0.0255 \\
Power Supply 1 & 0.480 & 0.0236 \\
Power Supply 2 & 0.719 & 0.0014 \\
Power Supply 3 & 0.914 & 0.0064 \\
\hline
\end{tabular}

measured data at higher harmonics and lower power levels. The weights used in the optimization routine ensures the extraneous harmonic angles are not modeled. It will be shown in the validation section that this does not effect accuracy of the model.

\subsection{Model Validation}

The parameter estimation procedure described in the previous section was validated in two ways. Firstly, the harmonic current magnitude and angles obtained from the DBFs and estimated parameters were used to construct time domain waveforms (frequency domain to time domain). The constructed waveforms were compared against time domain data obtained from device measurements.

Figure 7.4 shows the time domain waveform obtain frequency to time construction of the harmonic models for two converter power levels. The waveforms matched well with measured waveform. The waveforms align very well with the measurement data suggesting the model is able to correctly model the dominant harmonic angles. Reconstructed waveforms show slight mismatch at lower levels (top), which can be attributed to selection of weights for phase angle modeling. This should have minimal effect in a harmonic power flow simulation as the harmonic magnitude model is accurate. 


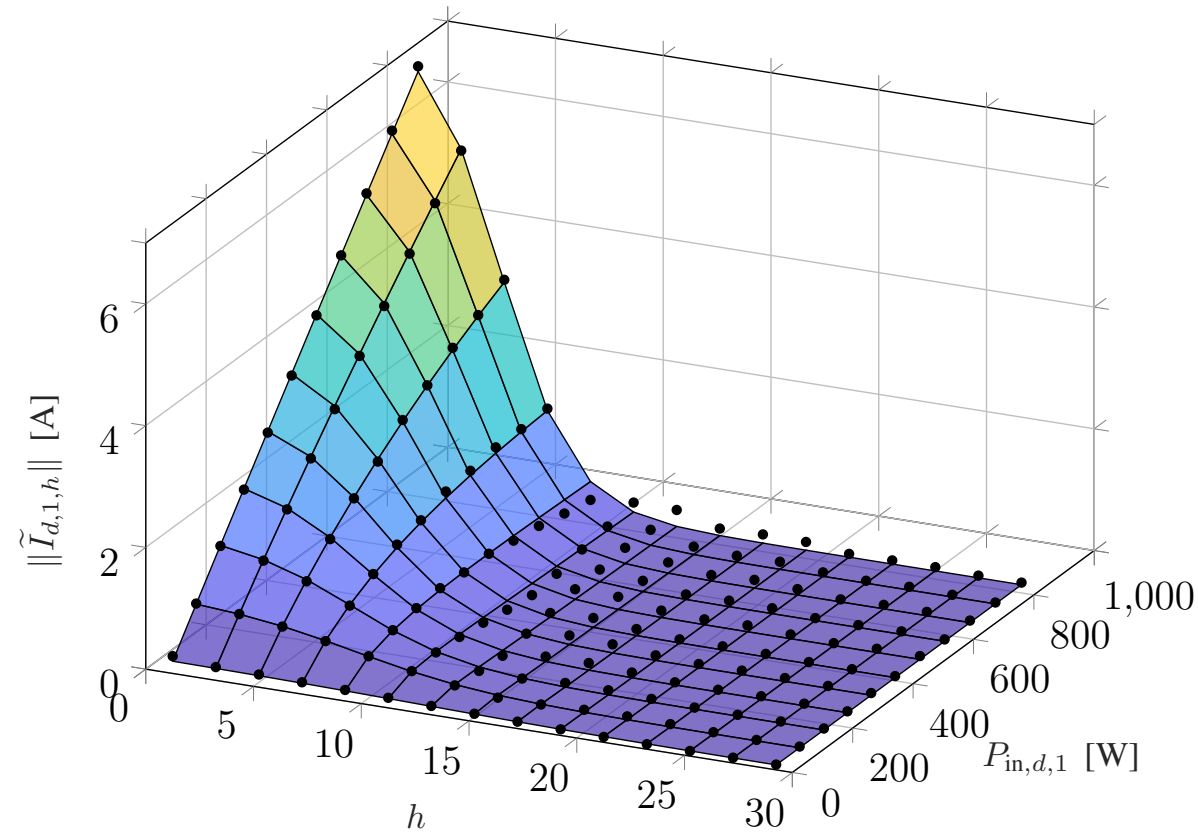

(a) Harmonic magnitude model fit (surface) along with measured data (black markers).

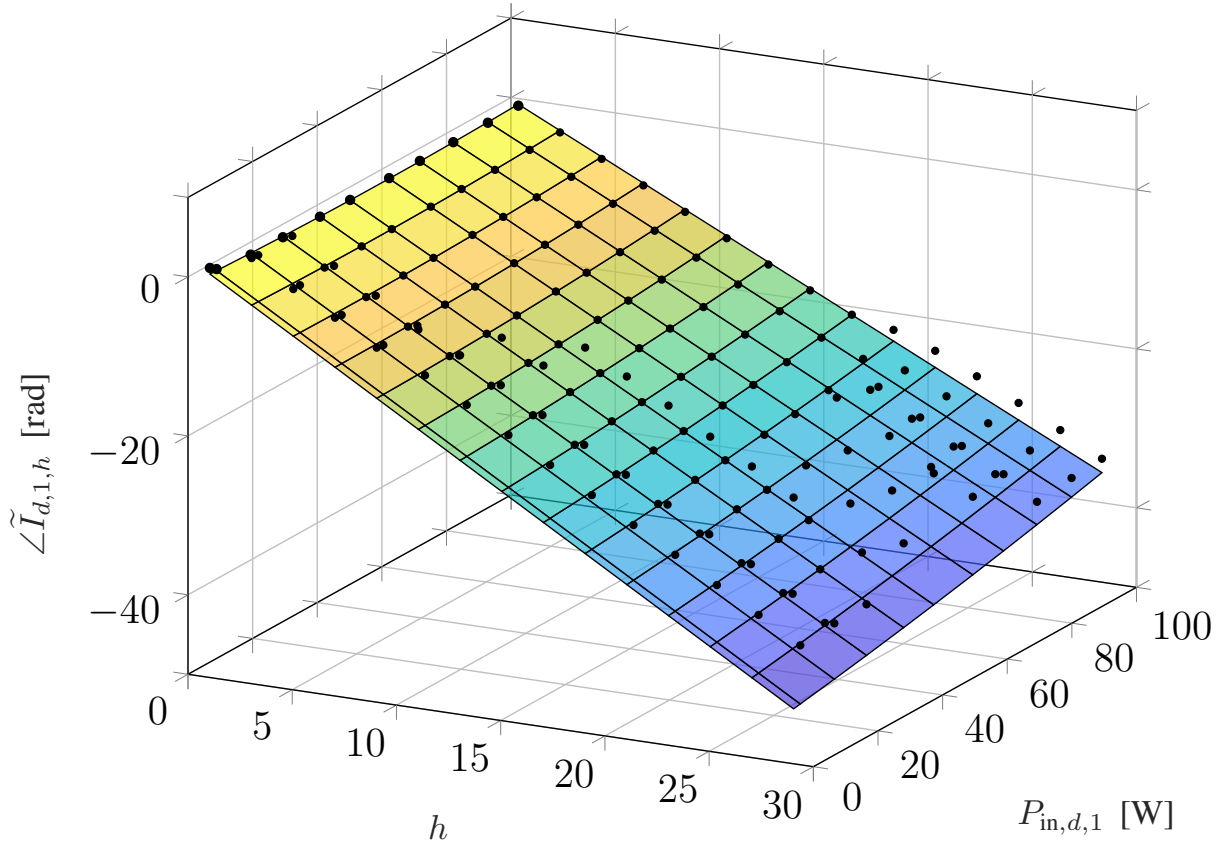

(b) Harmonic angle model fit (surface) along with measured data (black markers).

Figure 7.3: Device specific behavioral model (a) harmonic magnitude model fit (b) harmonic angle model fit, along with measured data (black markers)

Secondly, the proposed models and the estimated parameters were compared against empirical models (interpolation technique) for nonlinear converters in simulations using the BEEAM library. The proposed DBFs were able to correctly model converter harmonics and the converter power 

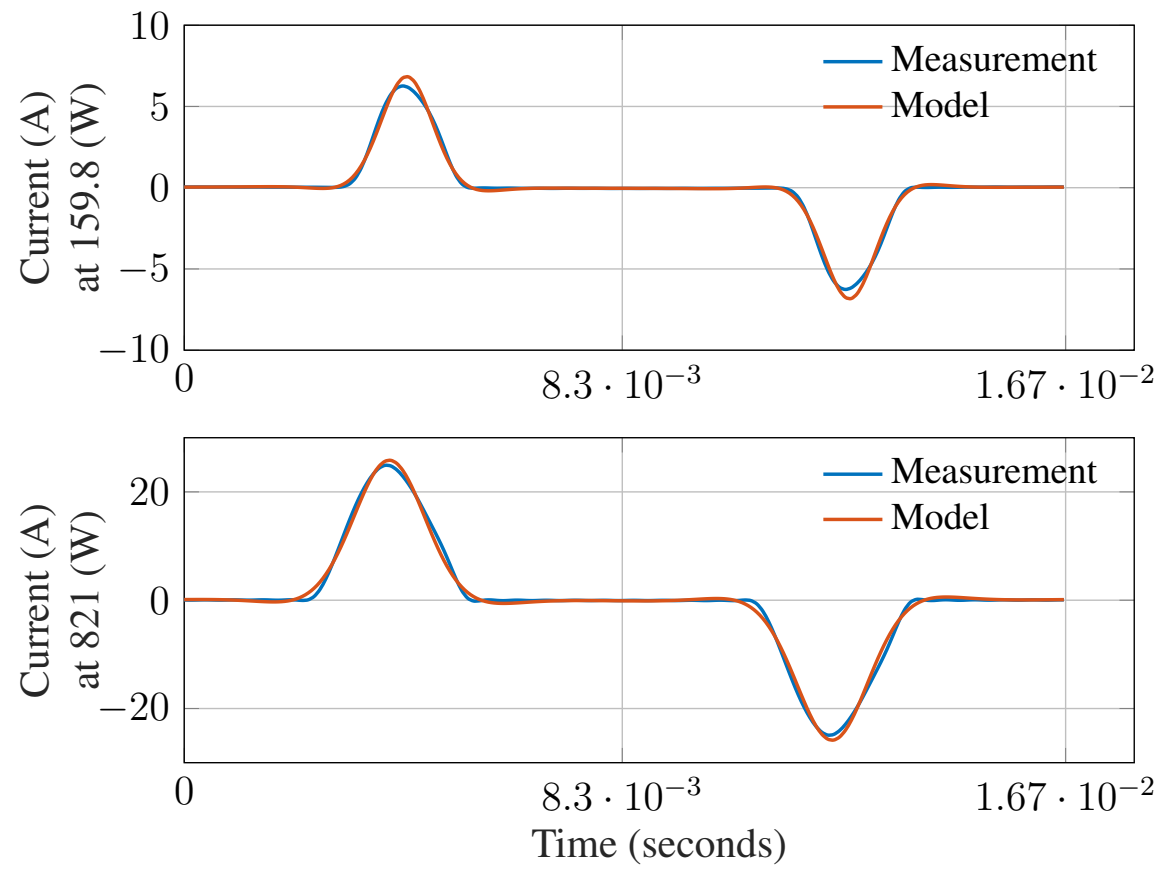

Figure 7.4: Validation result for device behavior model characterization for converter model operating at $160 \mathrm{~W}$ (top) and converter operating at $821 \mathrm{~W}$ (bottom).

consumption. Profiling simulation data form the two simulations yielded simulation time performance. Simulation using the empirical model took 0.135009 seconds, while simulation model using the proposed DBFs took 0.0475081 seconds. Therefore, a $64.8 \%$ improvement in simulation time performance. 


\section{Chapter 8}

\section{Toolkit Validation}

To evaluate the accuracy of the software developed in this work, simulations using the toolkit were compared to laboratory measurements on an experimental testbed constructed at the Powerhouse Energy Campus at Colorado State University. The following sections describe the laboratory experiments and comparison to simulation.

\subsection{Laboratory Experiments}

The experimental testbed was designed to emulate realistic loads found in a small office building, comprising laptop computer chargers, LED lighting systems, and miscellaneous DC and AC loads. The testbed also enabled both AC and DC configurations, under either balanced or unbalanced loading.

A notional diagram of the testbed is shown in Figure 8.1, 8.2 where Figure 8.1 represents the AC testbed connections and 8.2 DC distribution configuration. The testbed comprised a threephase $\Delta$-Y building transformer with three parallel load branches in both configurations; loads were connected to a single-phase or multiple phases. The primary side of the transformer, connected in $\Delta$, was tied to utility mains. Each load branch in the AC distribution case consisted of a parallel combination of one or more of the following devices: computer laptop AC/DC power supply with controllable loads, light emitting diode (LED) light fixture supplied by an AC/DC driver, AC/DC power supplies with controllable resistive loads, and resistive heating elements. In the DC configurations, the output voltage of the building transformer (the same used in the AC case) was converted to DC voltage with an AC/DC “central converter". Each DC load branch consisted of one or more of the following devices: computer laptop DC/DC power supply connected to a controllable load, light emitting diode (LED) light fixture, controllable load bank (CLB).

The physical testbed is shown in Figure 8.3. The testbed was constructed using industrial grade electrical equipment, mounted on a panel-board for convenience during configuration changes and 


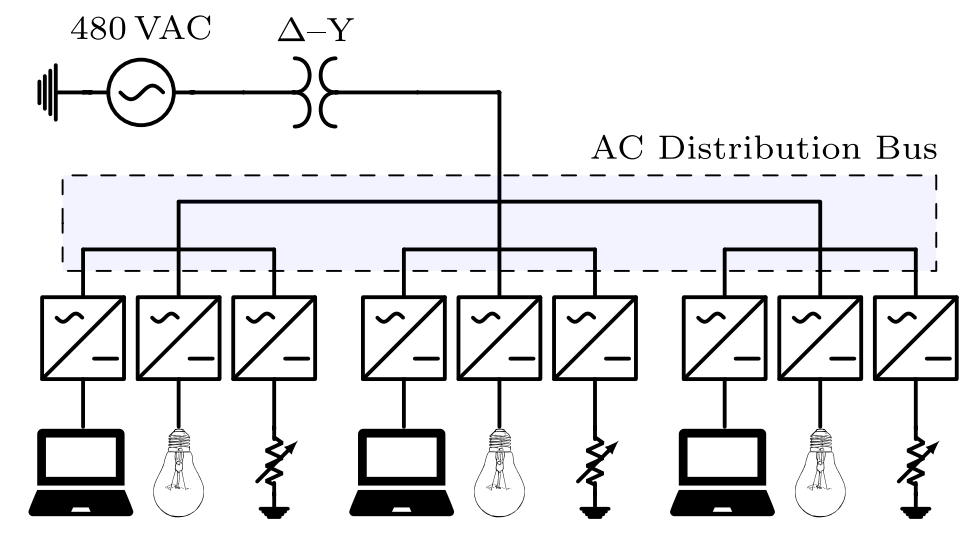

Figure 8.1: Notional diagram of testbed, AC configuration.

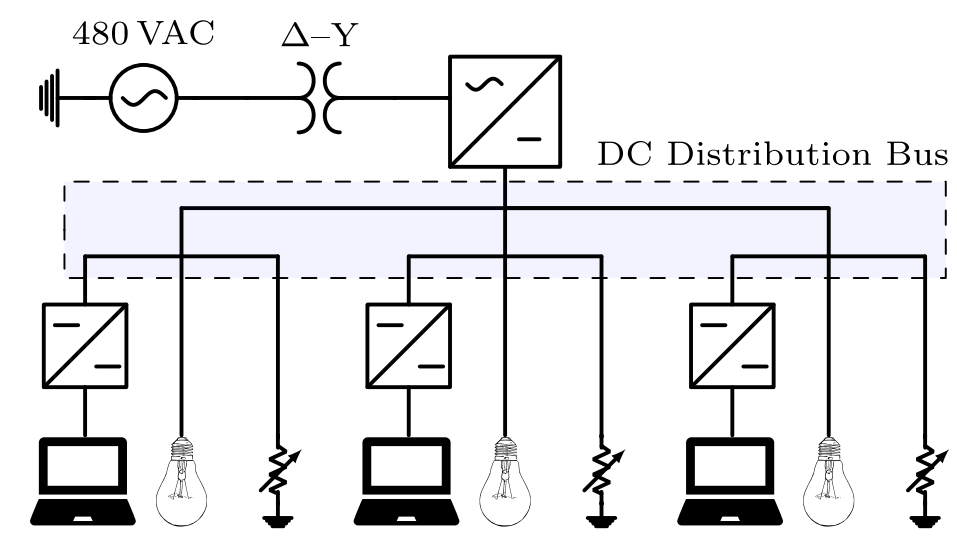

Figure 8.2: Notional diagram of testbed, DC configuration.

access to measurement points. To implement configuration changes and provide circuit protection, an electrical enclosure contained switches and fuses, supplying a total of six possible load branches.

The load center was connected to utility mains through a $3 \mathrm{kVA}, \Delta$-Y transformer (ACME, model T2A533081S), with a 480 V primary and 208/12 V secondary. Power devices in the testbed for the AC configuration consisted of: AC/DC converters, namely, Power Supply 1 (Mean Well, RSP-1000-48), Power Supply 2 (Xunbuma, T-1000-48V), Power Supply 3 (Mean Well, SE-100048), Laptop Charger 1 (HP, 391174-001), Laptop Charger 2 (HP, PA-1900-15C2), Laptop Charger 3 (HP, PA-1121-12H), LED Drivers 1-3 (Mean Well, APV-25-24). 


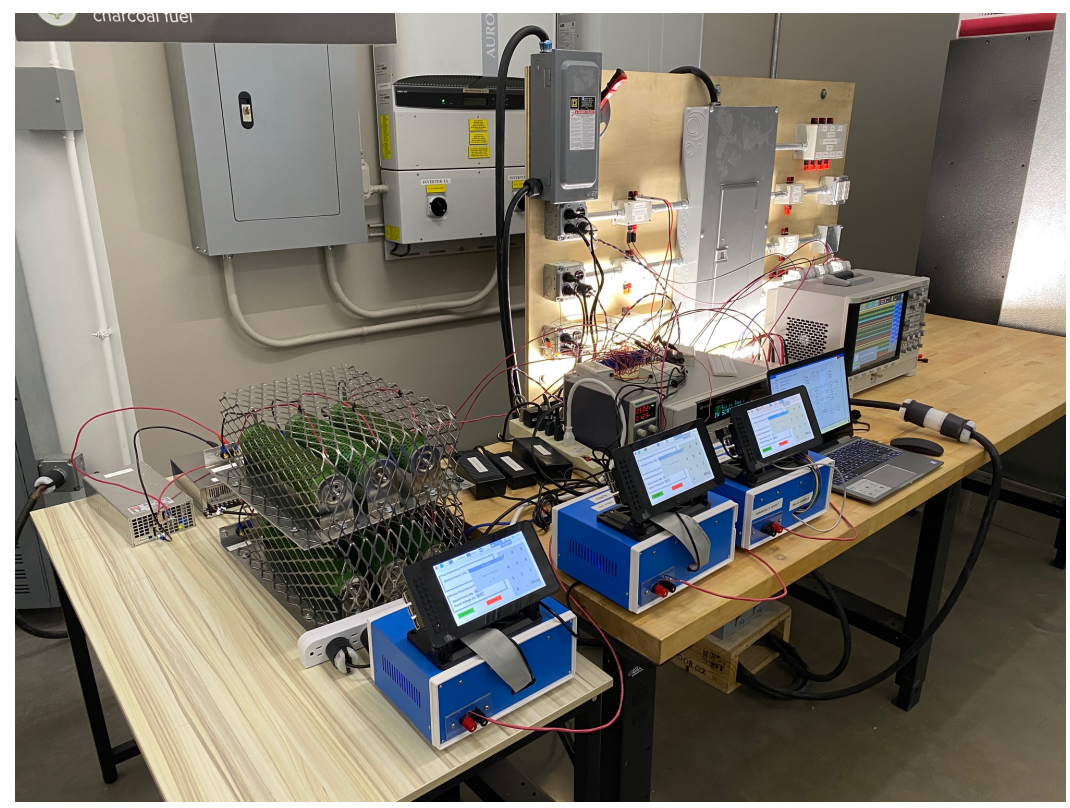

Figure 8.3: Testbed hardware with power analyzer, laptop, LED's and resistive loads banks.

For the DC configuration, the test setup consisted of a central converter that powers DC loads, model Nextek, PHD16-ACDC-DIM-P-24-6. It is a converter that is powered using Class 1 AC (90-305 V AC) or DC (127-431 V DC). The converter has 16 output ports rated at $24 \mathrm{~V} \mathrm{DC} \mathrm{(95}$ W). The DC loads consisted of, Laptop Chargers 3-6 (Bix Power, BX-DD90X-24V). End point electrical loads used in both configurations were, LEDs 1-3 (24 VDC, 22 W, 2500 lumens). 200$900 \mathrm{~W}$ (Lasko) heating elements, and three $400 \mathrm{~W}, 20 \mathrm{~V}$ (custom built) resistive controllable load banks.

Using six different wiring configurations and four loading scenarios, a set of 24 experimental scenarios were implemented and measured. Herein, each scenario is referred to by wiring configuration and load condition as "Scenario $w, l$ ", where $w \in\{1, \ldots, 6\}$ is the wiring configuration number and $l \in\{1, \ldots, 4\}$ is the loading configuration. When referring collectively to all loading conditions under a given winding configuration, the shorthand "Scenario w" is used. The AC configurations were used to obtain experimental measurements under 16 scenarios (Scenarios 1.1-1.4, 2.1-2.4, 3.1-3.4); the DC configuration was used for obtaining measurements under eight scenarios (Scenarios 5.1-5.4, 6.1-6.4). 
In Scenarios 1-2, power electronic loads on the secondary side of the transformer were connected as shown in Table 8.1. In these scenarios, the four loading values were applied on the converters as follows. The AC/DC power supplies were operated under loads of: no load, $10 \Omega$, $5.6 \Omega$, or $4 \Omega$. The laptop chargers were connected to controllable load banks, set to power levels specified below. The LED drivers were loaded with LEDs 1-3 or no load. Data was collected using Keysight multifunction switch measuring unit (MU) model 34980A with Keysight 34921T multiplexer and a Keysight PA2203A power analyzer.

Table 8.1: Device configurations for AC Scenarios 1-2.

\begin{tabular}{|l|c|c|c|}
\hline & Phase A & Phase B & Phase C \\
\hline Power Supply 1 & $\checkmark$ & & \\
\hline Power Supply 2 & & $\checkmark$ & \\
\hline Power Supply 3 & & & $\checkmark$ \\
\hline Laptop Charger 1 & $\checkmark$ & & \\
\hline Laptop Charger 2 & & $\checkmark$ & \\
\hline Laptop Charger 3 & & & $\checkmark$ \\
\hline LED Driver 1 & $\checkmark$ & & \\
\hline LED Driver 2 & & $\checkmark$ & \\
\hline LED Driver 3 & & & $\checkmark$ \\
\hline
\end{tabular}

Scenarios 3-4 were same as scenarios 1-2, except that the AC/DC power supplies and resistances were replaced by heaters at the same rated power level. The converter connections under these scenarios are shown in Table 8.2. Loads applied to the converters with respect to each phase in Scenarios 1-4 are shown in Table 8.3.

In the DC configuration for Scenarios 5-6, DC voltage was supplied by the external AC/DC laptop chargers, CLB 1-3 which were connected to DC/DC laptop charters 5-6, respectively, LEDs, and resistors. Scenario 5 was identical to Scenario 6 except that in Scenario 5, the central converter was supplied with $120 \mathrm{~V}$ on the AC side, while in Scenario 6 it was supplied with $208 \mathrm{~V}$, phase to phase connection. The four load power levels for each DC scenario are shown in Table 8.4. 
Table 8.2: Device configurations for AC Scenarios 3-4.

\begin{tabular}{|l|c|c|c|}
\hline & Phase A & Phase B & Phase C \\
\hline Laptop Charger 1 & $\checkmark$ & & \\
\hline Laptop Charger 2 & & $\checkmark$ & \\
\hline Laptop Charger 3 & & & $\checkmark$ \\
\hline LED Driver 1 & $\checkmark$ & & \\
\hline LED Driver 2 & & $\checkmark$ & \\
\hline LED Driver 3 & & & $\checkmark$ \\
\hline
\end{tabular}

Table 8.3: Load power settings for hardware verification tests, AC scenario 1-4.

\begin{tabular}{cccc}
\hline \multicolumn{5}{c}{ Loads for Scenario 1, 3 (Balanced) } \\
\hline Scenario & Phase A [W] & Phase B [W] & Phase C [W] \\
\hline $1.1,3.1$ & 0 & 0 & 0 \\
$1.2,3.2$ & 100 & 100 & 100 \\
$1.3,3.3$ & 300 & 300 & 300 \\
$1.4,3.4$ & 500 & 500 & 500 \\
\hline \multicolumn{5}{c}{ Loads for Scenario 2,4 (Unbalanced) } \\
\hline \multicolumn{5}{c}{ Scenario } & Phase A [W] & Phase B [W] & Phase C [W] \\
\hline $2.1,4.1$ & 0 & 0 & 0 \\
$2.2,4.2$ & 700 & 100 & 700 \\
$2.3,4.3$ & 300 & 500 & 700 \\
$2.4,4.4$ & 700 & 500 & 0 \\
\hline
\end{tabular}

\subsection{Comparison of Toolkit Results with Measurements}

To evaluate the accuracy of predicted electrical efficiency using the toolkit, simulations of the experimental scenarios described above were performed using the BEEAM toolkit and compared to experimental measurements. Predicted total system efficiencies accumulated across all devices in the network are compared to measured system efficiencies for Scenarios 1-2, 3-4, and 5-6. Figures 8.6-8.8, respectively. As shown in Figures 8.6-8.8, total system efficiency predicted by the toolkit matched well with efficiencies over all scenarios. Predicted total system power losses in the network in Scenarios 1-2, 3-4, and 5-6 are shown in Figures 8.9-8.11, respectively; the simulated 
Table 8.4: Load power settings for hardware verification tests, DC scenario 5-6.

\begin{tabular}{cc}
\hline Scenario & Power [W] \\
\hline $5.1 \& 6.1$ & 0 \\
$5.2 \& 6.2$ & 178 \\
$5.3 \& 6.3$ & 541 \\
$5.4 \& 6.4$ & 904 \\
\hline
\end{tabular}

total power losses show reasonably good agreement with the experimental measurements over all scenarios. Figure 8.4 shows the Modelica simulation model setup for the unbalanced AC scenario (Scenario 2.3).

Measurements are performed using transducers that exhibit uncertainty as a result of inherent physical properties. The error bars in the bar plots show the associated uncertainty resulting from measurements performed during the laboratory experiments. A detailed treatment of the uncertainty analysis can be found in [62].

Figure 8.5 shows the simulation model setup for the DC Scenario 5.4. Loss models for the DC/DC converters are passed on to the DC/DC converter models using Modelica record. The DC distribution setup consists of three DC/DC converters and other DC loads connected directly to the central converter. To simplify simulation setup, the DC loads connected directly to the central converter are lumped together in LumpedDCLoads model. Notice the red colored cross marks on the secondary of the transformer. These are terminal blocks for an unbalanced transformer connection with open circuited B and C phases. The central converter is connected on phase A.

Discussion: Comparison of predicted versus measured efficiencies and power losses in the initial toolkit implementation revealed that the system- and device-level efficiencies could be predicted with reasonable accuracy in AC and DC configurations in both AC and DC configurations in both balanced and unbalanced conditions. However, on an absolute wattage basis, further improvements to the underlying component models were identified.

Discrepancies between simulated and measured powers were likely attributable to simplifying assumptions made in the initial toolkit implementation. One simplification was in the building 


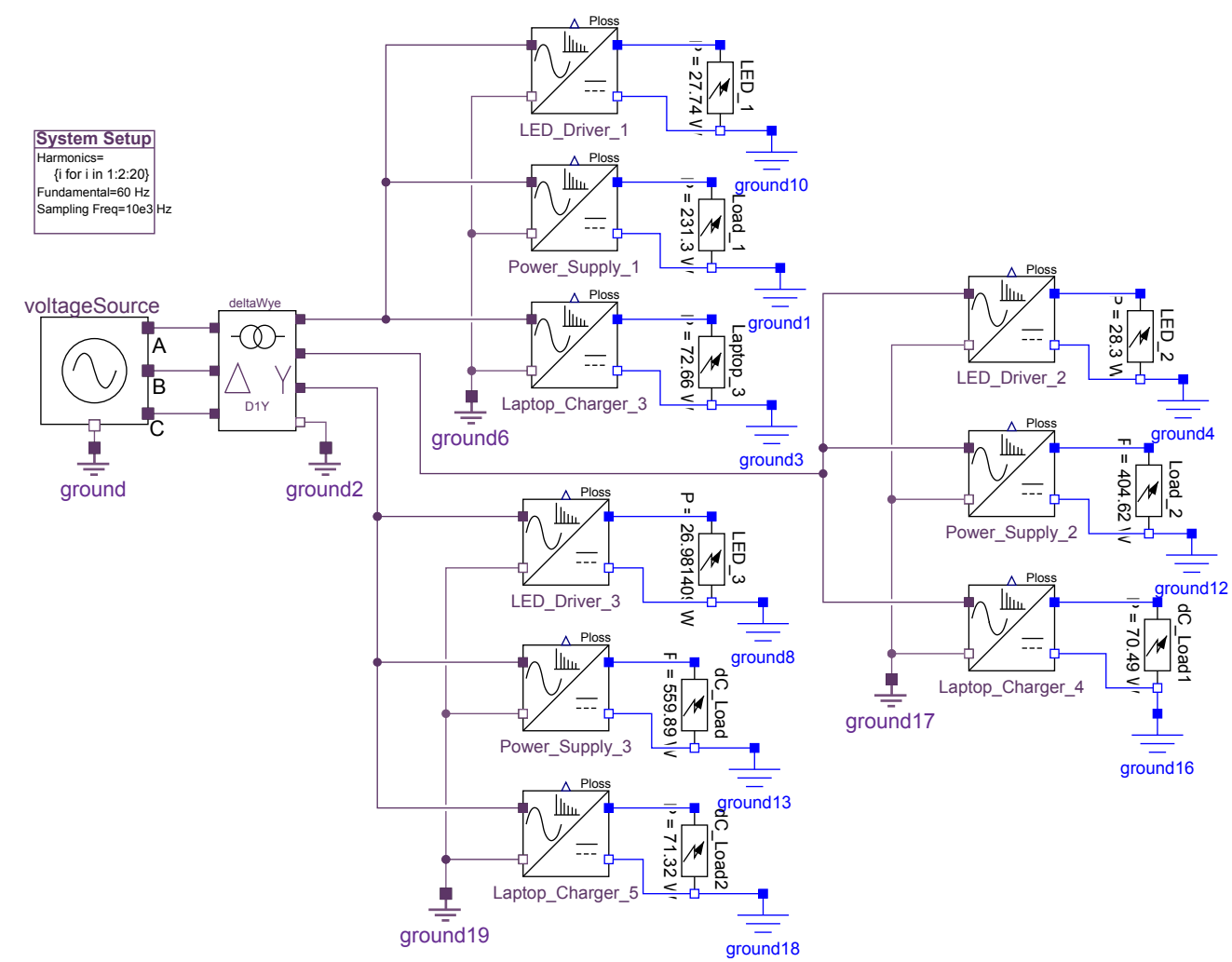

Figure 8.4: Graphical representation of example electrical network in BEEAM, corresponding to AC distribution Scenario 2.3.

transformer model used in the validation studies. In particular, the balanced linear three-phase transformer modeling approach depicted in Figure 4.4 was used for simplicity. This model assumes the secondary load is balanced and consequently, neglects potential coupling between phase currents and their harmonics. Second, it was assumed that the transformer was linear, with parameters extracted from measurements taken under purely sinusoidal, non-magnetically saturation conditions. In future work, the transformer model will be improved by accounting for load imbalance and phase coupling using a symmetrical components model. 


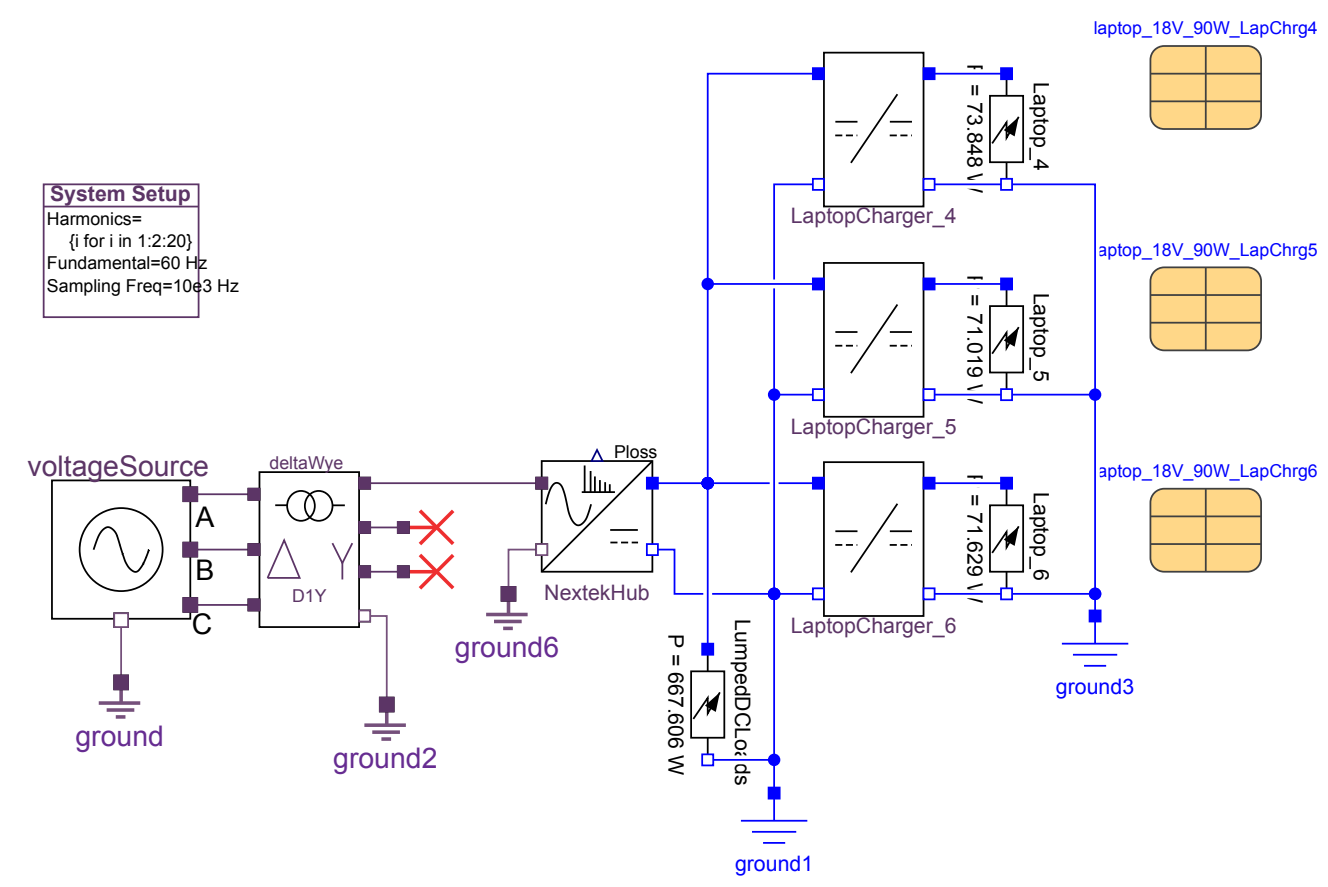

Figure 8.5: Graphical representation of example electrical network in BEEAM, corresponding to AC distribution Scenario 5.4.

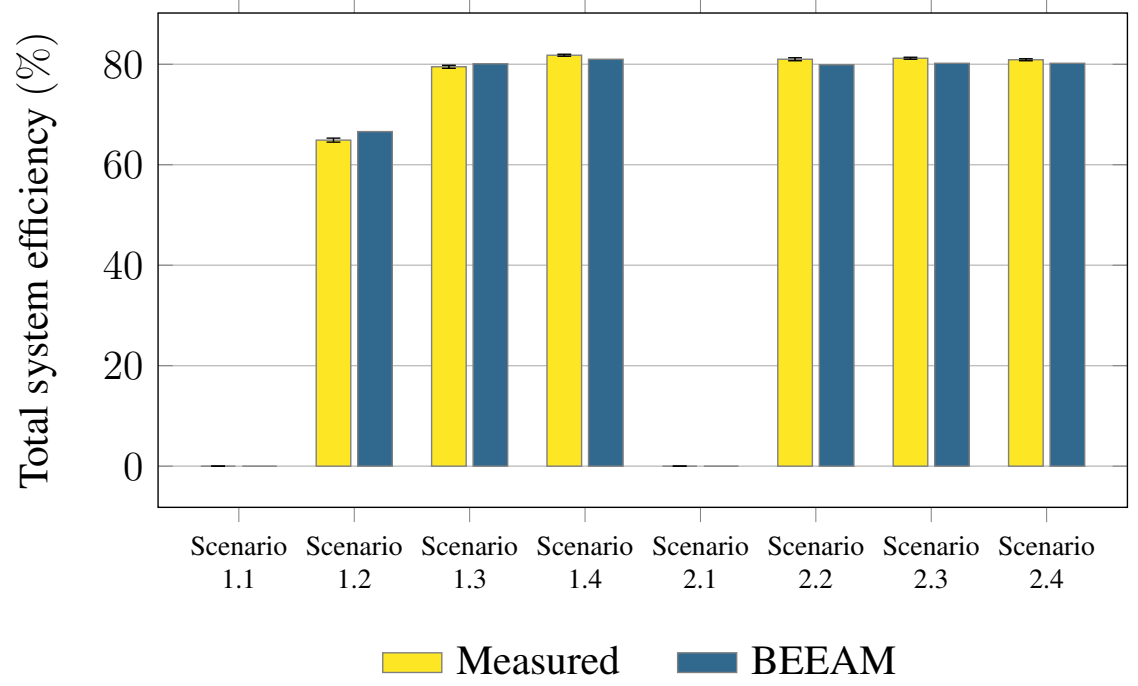

Figure 8.6: Predicted versus measured total system efficiencies, Scenarios 1-2. 


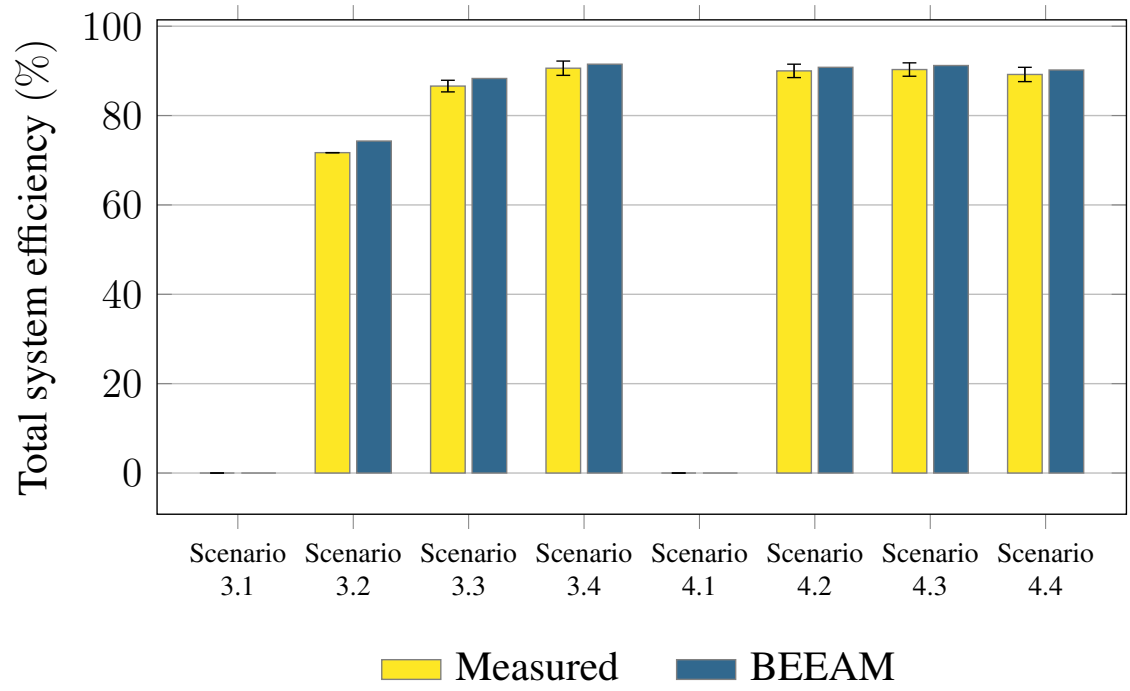

Figure 8.7: Predicted versus measured total system efficiencies, Scenarios 3-4.

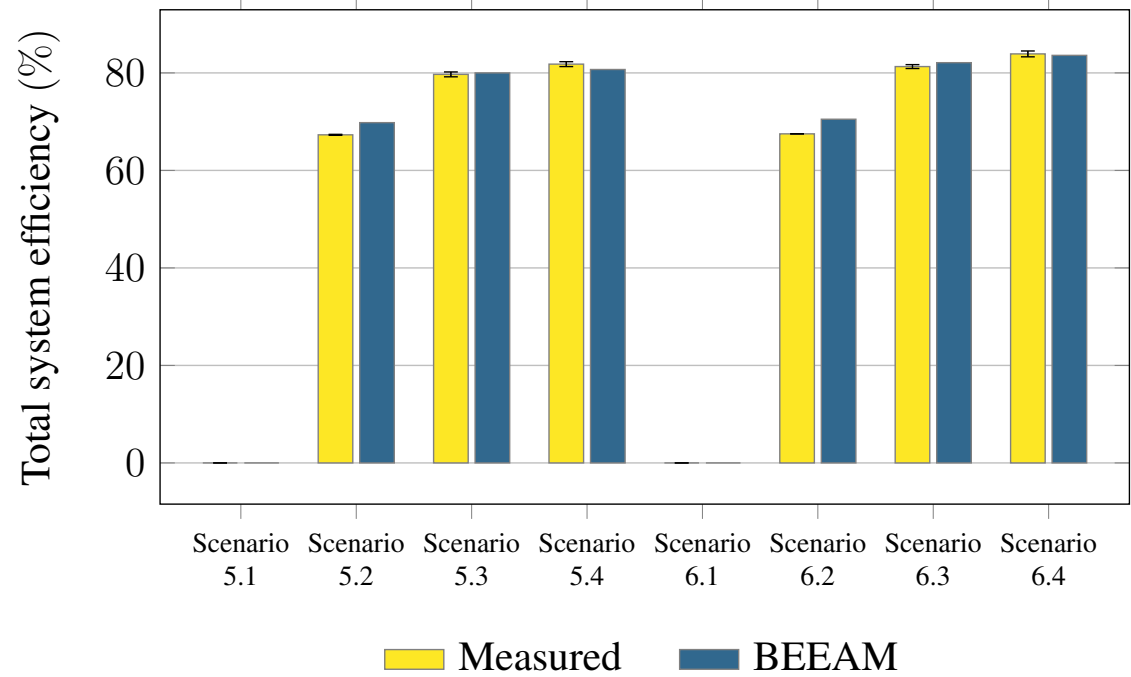

Figure 8.8: Predicted versus measured total system efficiencies, Scenarios 5-6 


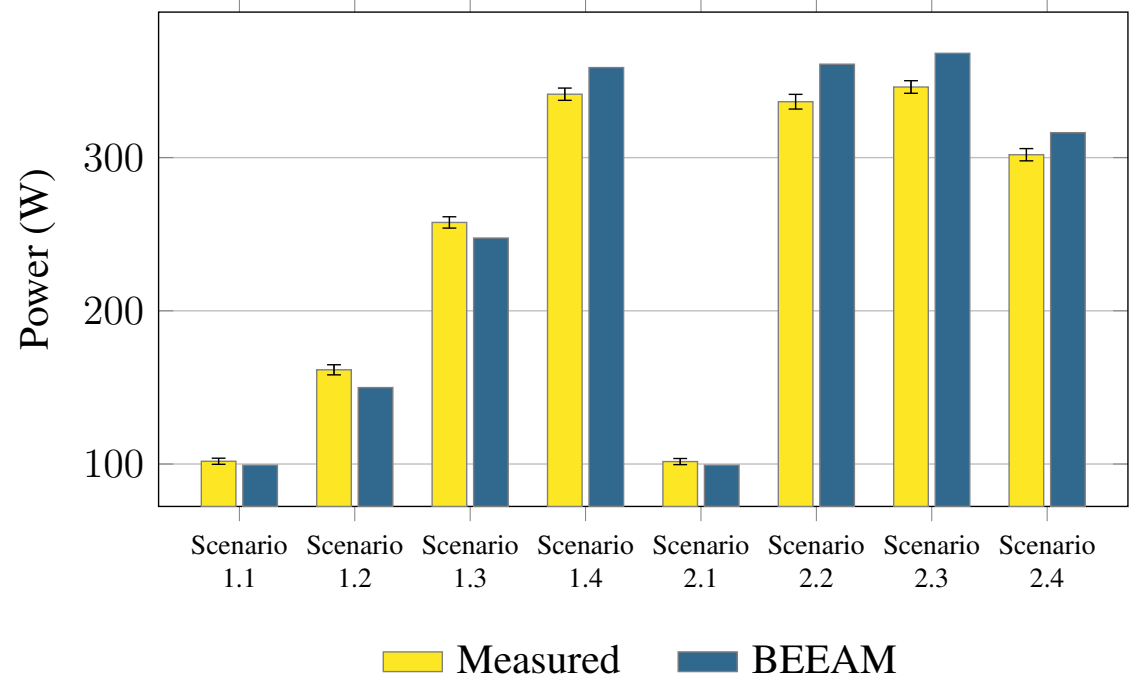

Figure 8.9: Predicted versus measured total system power losses, Scenarios 1-2.

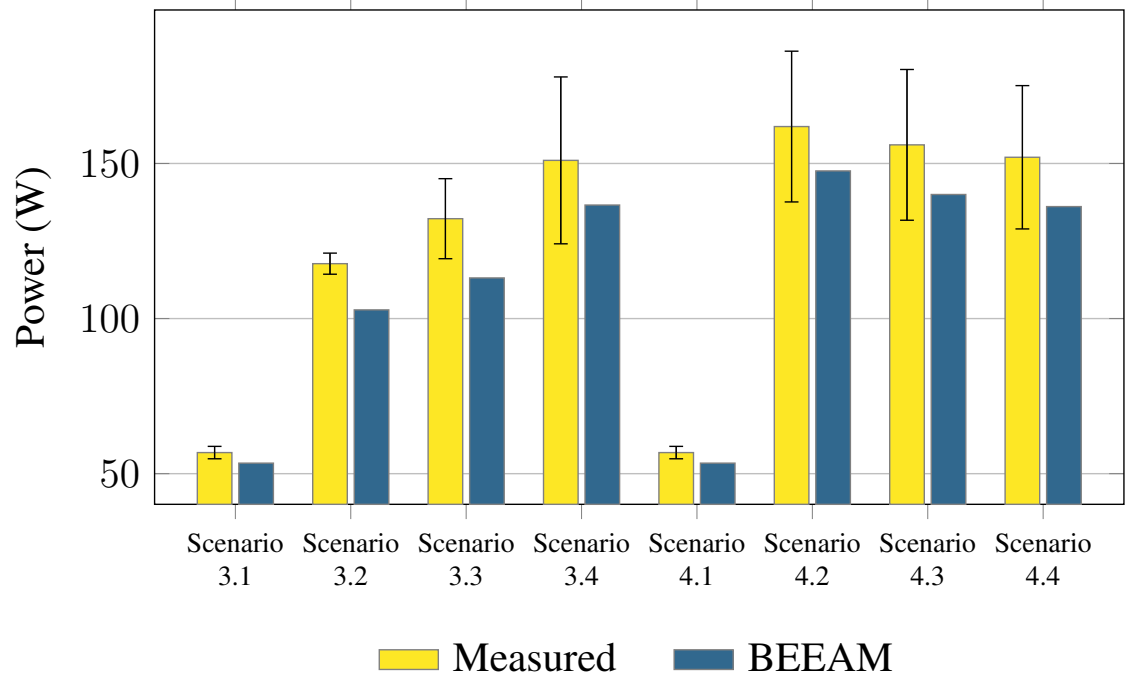

Figure 8.10: Predicted versus measured total system power losses, Scenarios 3-4. 


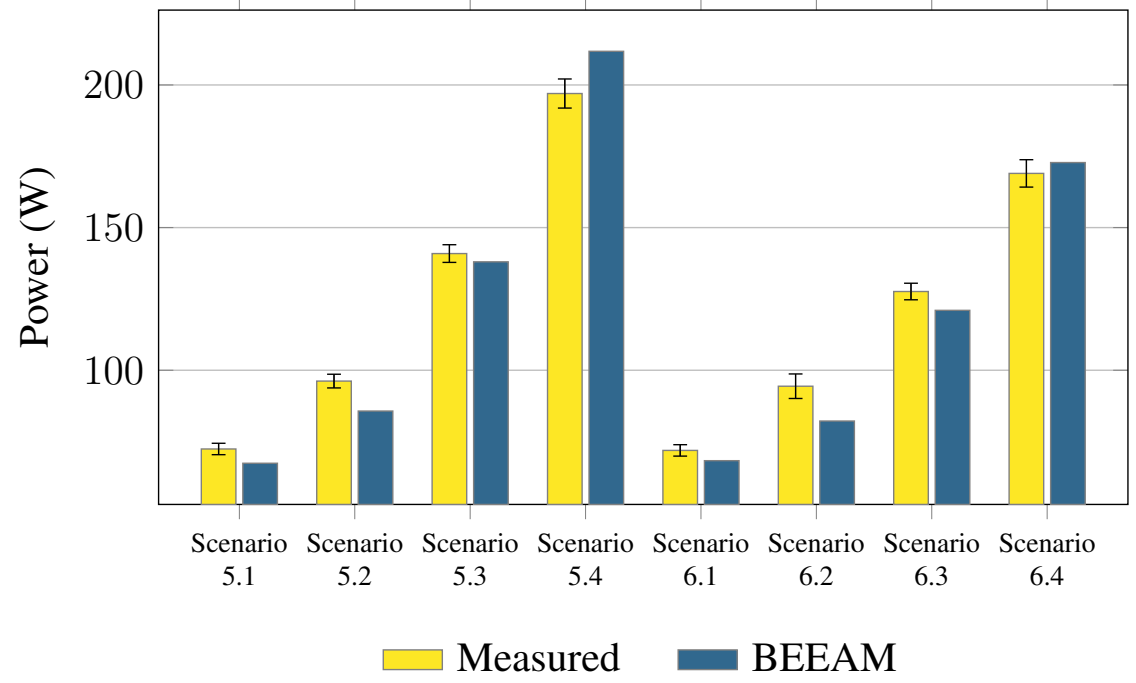

Figure 8.11: Predicted versus measured total system power losses, Scenarios 5-6. 


\section{Chapter 9}

\section{Summary and Suggestions for Future Work}

This dissertation described a modeling framework and an open source software toolkit for comparing AC, DC and hybrid AC/DC electrical distribution systems. Major contributions of this work are: description of a frequency domain modeling framework for distributions using one- and two-port network theory that accounts for harmonics in voltages and currents, software toolkit BEEAM [13] developed in an object-oriented multi-domain modeling language (Modelica), characterization of closed form device-specific behavioral functions for modeling nonlinear device harmonics.

Chapter 3 and 4 laid the foundation for the modeling framework and described the one- and two-port network framework. This was followed by application of the modeling framework using harmonic power flow on a distribution system containing nonlinear load. The example illustrated key concepts such as network formulation and solution using an iterative numerical solver. Chapter 6 described the modeling toolkit developed in Modelica. The chapter describes important Modelica language features incorporated in the library design, such as a new connector class for the AC distribution harmonics modeling.

Chapter 8 showed the experimental setup and validation studies by comparing predictions from the BEEAM library models with laboratory measurements. Comparison of predicted versus measured efficiencies and power losses in the initial toolkit implementation revealed that device- and system-level efficiencies could be predicted with reasonably good accuracy under both balanced and unbalanced AC scenarios. A detailed uncertainty analysis also demonstrated that the maximum estimated error for system efficiency across all scenarios was 3\% and that the measured and modeled system efficiency agreed with the experimental uncertainty in approximately half the scenarios.

The multi-domain simulation capabilities of the BEEAM library were demonstrated in a cosimulation setup with Energy+ [63] [64]. The toolkit provides the flexibility of a graphical user 
interface that results in small learning curve and easy adoption for new users, while at the same time allows for access to underlying Modelica code for more experienced users. The BEEAM library has been released under a permissive open source license. More information can be found in Appendix B.3.

Some limitations in the research were identified. Firstly, the initial validation studies were performed on a small-scale demonstration testbed that did not include some devices that could be found in modern buildings, such as distributed generator and electrical motor drives. Secondly, a balanced, linear transformer was used in the validation studies that neglected potential coupling and harmonics between electrical phases in the transformer or any nonlinearities.

The BEEAM library, with its modeling framework based on harmonic power flow, has been found to provide a suitable balance between simulation speed and accuracy when compared to conventional electrical distribution modeling techniques such as a time domain simulation and traditional power flow. This dissertation does not directly address the issue of scalability or numerical stability of the underlying BEEAM solver for systems larger than the studies performed in this research; scalability and stability of the toolkit are topics of suggested future research. However, BEEAM has been used to simulate a system of approximately 5,000 states, executing in $0.15 \mathrm{~s}$. These initial results indicate that the BEEAM toolkit can simulate systems of at least moderate size, capturing power flows and harmonic content, with increased computational efficiency compared to full time-domain simulation.

Lastly, though the closed form device-specific behavior function, described in chapter 7, was able to accurately model the converter harmonics, some limitations were observed. Power factor correction (PFC) control is an active control technique that corrects the power factor of a rectifier by actively shaping the input current into a sinusoidal waveform that is in phase with the input voltage. As was shown in the characterization studies, the proposed model for the harmonic current magnitude was able to correctly model harmonics at all harmonic frequencies and power levels. However, it was observed that certain converters, the PFC was operational only for higher power 
levels resulting in harmonic spectra with discontinuities. Such a converter cannot be modeled using the proposed DBF and one must revert to the interpolation method.

\subsection{Suggestions for Future work}

This dissertation provides a description of a modeling framework and the development of a software toolkit library for modeling AC and DC distributions using harmonic power flow. Focus of this dissertation was on modeling nonlinear AC/DC converters and efficiency comparison of AC versus DC electrical distributions using simulation models built using the toolkit library. While the dissertation has covered all the research goals, the presented work can be improved further and expanded. This includes some of the tasks outlined in the preliminary dissertation that were not accomplished as a result of project time constraints. These tasks were, improved transformer modeling and building efficiency studies. Suggestions for future work are as follows:

- Utilize the library toolkit in efficiency studies of buildings with applications in lighting loads modeling.

- Improved transformer model that allow the removal of the assumption of a balanced, linear transformer by using a full symmetrical component representation. More research is needed into nonlinear harmonic models of the transformer, taking into account the effect of transformer core saturation and nonlinearities.

- Develop distribution equipment models, such as, harmonic cable models, capacitor banks, HVAC systems, and electrical machines.

- Latest addition to the library is the grouped converter model. Multiple converters connected in parallel are modeled using a single converter that scales the converter currents. Potential limitation of the model would be an over estimation of the system losses as the model does not account for harmonic cancellation as a result of variations in converter harmonic spectra [46]. An improved grouped converter model would implement effects from diversity and attenuation. 
Availability of a modeling platform, especially in a standardized language, is highly effective in furthering research and knowledge exchange. The SPICE program is a good example of such standardized simulation platform. The amount of research in electrical device models using the SPICE can be gauged by a search on the IEEE database for 'spice model' that shows more than ten thousand search result hits. The author hopes that the BEEAM library serves as a similar platform that spurs further research into the field of harmonic analysis and device harmonic models. 


\section{Bibliography}

[1] Vagelis Vossos, Karina Garbesi, and Hongxia Shen. Energy savings from direct-DC in U.S. residential buildings. Energy and Buildings, 68:223-231, Jan. 2014.

[2] Daniel L Gerber, Vagelis Vossos, Wei Feng, Chris Marnay, Bruce Nordman, and Richard Brown. A simulation-based efficiency comparison of AC and DC power distribution networks in commercial buildings. Applied Energy, 210:1167-1187, Jan. 2018.

[3] Stephen M Frank and Steffen Rebennack. Optimal design of mixed AC-DC distribution systems for commercial buildings: A Nonconvex Generalized Benders Decomposition approach. European Journal of Operational Research, 242(3):710-729, Feb. 2015.

[4] Brock Glasgo, Inês Lima Azevedo, and Chris Hendrickson. How much electricity can we save by using direct current circuits in homes? Understanding the potential for electricity savings and assessing feasibility of a transition towards DC powered buildings. Applied Energy, 180:66-75, Jan. 2016.

[5] Laurens Mackay, Nils H van der Blij, Laura Ramirez-Elizondo, and Pavol Bauer. Toward the Universal DC Distribution System. Electric Power Components and Systems, 45(10):10321042, Jan. 2017.

[6] R Weiss, L Ott, and U Boeke. Energy efficient low-voltage DC-grids for commercial buildings. In 2015 IEEE First International Conference on DC Microgrids (ICDCM), pages 154$158,2015$.

[7] U Boeke and M Wendt. DC power grids for buildings. In 2015 IEEE First International Conference on DC Microgrids (ICDCM), pages 210-214, 2015.

[8] K Shenai and K Shah. Smart DC micro-grid for efficient utilization of distributed renewable energy. In IEEE 2011 EnergyTech, pages 1-6, 2011. 
[9] Brinda A Thomas, Inês L Azevedo, and Granger Morgan. Edison Revisited: Should we use DC circuits for lighting in commercial buildings? Energy Policy, 45:399-411, Jan. 2012.

[10] Karina Garbesi, Vagelis Vossos, and Shen Hongzia. Catalog of DC Appliances and Power Systems. Technical report, Lawrence Berkeley National Laboratories, Mar. 2011.

[11] Frank, Stephen. Optimal design of mixed AC-DC distribution systems for commercial buildings. Dissertation, Colorado School of Mines, Golden, CO, 2013.

[12] F Zhang, C Meng, Y Yang, C Sun, C Ji, Y Chen, W Wei, H Qiu, and G Yang. Advantages and challenges of DC microgrid for commercial building a case study from Xiamen university DC microgrid. In 2015 IEEE First International Conference on DC Microgrids (ICDCM), pages $355-358,2015$.

[13] National Renewable Energy Laboratory (NREL) Github code repository. Building electrical efficiency analysis model (BEEAM). [Online] Available: https://github.com/NREL/BEEAM.

[14] Robert W. Erickson. Fundamentals of power electronics. Kluwer Academic, Norwell, Massachusetts, second edition. edition, 2001.

[15] James Frederick Lazar. Analysis of single phase rectifier circuits. Phd dissertation, California Institute of Technology, 1997.

[16] Karen George. Dc power production, delivery and utilization. Electric Power Research Institute White Paper, 2006.

[17] Paul Savage, Robert R Nordhaus, and Sean P Jamieson. DC Microgrids: Benefits and Barriers. Technical report, Yale School of Forestry \& Environmental Studies, Jan. 2010.

[18] Guy AlLee and William Tschudi. Edison redux: $380 \mathrm{vdc}$ brings reliability and efficiency to sustainable data centers. 10(6):50-59. Conference Name: IEEE Power and Energy Magazine.

[19] J. Duncan. Glover. Power system analysis \& design. Cengage Learning, Boston, MA, 2015. 
[20] Luigi Gentile Polese, Stephen Frank, Mahmoud Alahmad, Keun Lee, and Clarence Waters. Modeling and power efficiency analysis of building electrical distribution systems. Technical Report TP-5500-52657, National Renewable Energy Laboratory, Golden, CO, USA, September 2011.

[21] A. Santos, J. Cale, A. Othee, D. Gerber, S. Frank, G. Duggan, and D. Zimmerle. Comparison of load models for estimating electrical efficiency in dc microgrids. In Proceedings of the $3 r d$ IEEE International Conference on DC Microgrids, 2019, Matsue, Japan, 2019.

[22] Waldemar Rebizant, Janusz Szafran, and Andrzej Wiszniewski. Digital Signal Processing in Power System Protection and Control. Signals and Communication Technology. SpringerVerlag.

[23] Alan V. Oppenheim. Signals \& systems. Prentice-Hall signal processing series. Prentice Hall, Upper Saddle River, N.J, 2nd ed. edition, 1997.

[24] Daozhi Xia and G. T. Heydt. Harmonic Power Flow Studies, Part I Formulation and Solution. IEEE Power Engineering Review, PER-2(6):17-17, June 1982. Conference Name: IEEE Power Engineering Review.

[25] Daozhi Xia and G. T. Heydt. Harmonic Power Flow Studies - Part II Implementation and Practical Application. IEEE Transactions on Power Apparatus and Systems, PAS101(6):1266-1270, June 1982. Conference Name: IEEE Transactions on Power Apparatus and Systems.

[26] W. Xu, J. R. Marti, and H. W. Dommel. A multiphase harmonic load flow solution technique. IEEE Transactions on Power Systems, 6(1):174-182, February 1991.

[27] L. Sainz, J. Clua, and O. Jordi. Load modeling for unbalanced harmonic power flow studies. In 8th International Conference on Harmonics and Quality of Power. Proceedings (Cat. No.98EX227), volume 2, pages 665-671 vol.2, October 1998. 
[28] A. Ulinuha, M.A.S. Masoum, and S.M. Islam. Harmonic power flow calculations for a large power system with multiple nonlinear loads using decoupled approach. In 2007 Australasian Universities Power Engineering Conference, pages 1-6, December 2007.

[29] C. N. Bathurst, B. C. Smith, N. R. Watson, and J. Arrillaga. A modular approach to the solution of the three-phase harmonic power-flow. 15(3):984-989.

[30] Ewald Fuchs and Mohammad A. S. Masoum. Power Quality in Power Systems and Electrical Machines. Academic Press, July 2015.

[31] Marko Jereminov, Amritanshu Pandey, David M. Bromberg, Xin Li, Gabriela Hug, and Larry Pileggi. Steady-state analysis of power system harmonics using equivalent split-circuit models. In 2016 IEEE PES Innovative Smart Grid Technologies Conference Europe (ISGTEurope), pages 1-6, October 2016. ISSN: null.

[32] G. T. Heydt and W. M. Grady. The HARMFLO code: Version 4. 0: User's guide. Technical Report EPRI-EL-4920-CCM, Purdue Univ., Lafayette, IN (USA). Dept. of Electrical Engineering; Electric Power Research Inst., Palo Alto, CA (USA), November 1986.

[33] William Mack Grady. Harmonic power flow studies. PhD thesis, Purdue University, January 1983.

[34] Harmonic Analysis Software I Power Quality Software I Harmonics. Available: https://etap. com/product/harmonic-analysis-software.

[35] Functional mock-up interface. Available: https://fmi-standard.org/.

[36] Roger C. Dugan and Thomas E. McDermott. An open source platform for collaborating on smart grid research. In 2011 IEEE Power and Energy Society General Meeting, pages 1-7, July 2011. ISSN: 1944-9925.

[37] EPRI I Smart Grid Resource Center > Simulation Tool - OpenDSS. Available: https: //smartgrid.epri.com/SimulationTool.aspx. 
[38] W Grady and A. Mansoor. The potential effects of single-phase power electronic-based loads on power system distortion and losses, volume 1: Current harmonics produced by distributed single-phase power electronic loads.

[39] B.C. Smith, N.R. Watson, A.R. Wood, and J. Arrillaga. Newton solution for the steady-state interaction of AC/DC systems. Transmission and Distribution IEE Proceedings - Generation, 143(2):200-210, March 1996. Conference Name: Transmission and Distribution IEE Proceedings - Generation.

[40] M. Fauri. Harmonic modelling of non-linear load by means of crossed frequency admittance matrix. IEEE Transactions on Power Systems, 12(4):1632-1638, November 1997.

[41] Yuanyuan Sun, Guibin Zhang, Wilsun Xu, and Julio G. Mayordomo. A Harmonically Coupled Admittance Matrix Model for AC/DC Converters. IEEE Transactions on Power Systems, 22(4):1574-1582, November 2007. Conference Name: IEEE Transactions on Power Systems.

[42] E. Thunberg and L. Soder. A Norton approach to distribution network modeling for harmonic studies. IEEE Transactions on Power Delivery, 14(1):272-277, January 1999.

[43] C. F. M. Almeida and N. Kagan. Harmonic coupled norton equivalent model for modeling harmonic-producing loads. In Proceedings of 14th International Conference on Harmonics and Quality of Power - ICHQP 2010, pages 1-9, September 2010.

[44] M. E. Balci, D. Ozturk, O. Karacasu, and M. H. Hocaoglu. Experimental verification of harmonic load models. In 2008 43rd International Universities Power Engineering Conference, pages $1-4$, September 2008 .

[45] Duk-Gyoo Kim, Tatsuhito Nakajima, and Eisuke Masada. Harmonic analysis of a capacitorfiltered rectifier with line impedance. Electronics and Communications in Japan (Part I: Communications), 72(4):1-9, 1989. 
[46] A. Mansoor, W. M. Grady, A. H. Chowdhury, and M. J. Samotyi. An investigation of harmonics attenuation and diversity among distributed single-phase power electronic loads. IEEE Transactions on Power Delivery, 10(1):467-473, January 1995.

[47] Roland E. Thomas and Albert J. Rosa. Network Topology: A Supplement to Accompany the 3rd Edition of the Analysis and Design of Linear Circuits. John Wiley \& Sons Inc.

[48] Cale, J. and Sudhoff, S. and Chan, R. A field-extrema hysteresis model for ferrimagnetic materials. IEEE Transactions on Magnetics, 44(7):1728-1736, 2008.

[49] D Fregosi, S Ravula, D Brhlik, J Saussele, S Frank, E Bonnema, J Scheib, and E Wilson. A comparative study of DC and AC microgrids in commercial buildings across different climates and operating profiles. In 2015 IEEE First International Conference on DC Microgrids (ICDCM), pages 159-164, 2015.

[50] D.L. King, G. Gonzalez, M. Galbraith, and W.E. Boyson. Performance Model for GridConnected Photovoltaic Inverters. Technical report, Sandia National Laboratories, Sep. 2007.

[51] Torsten Blochwitz, Martin Otter, Martin Arnold, Constanze Bausch, Christoph Clauß, Hilding Elmqvist, Andreas Junghanns, Jakob Mauss, Manuel Monteiro, Thomas Neidhold, Dietmar Neumerkel, Hans Olsson, Jörg-Volker Peetz, and Susann Wolf. The functional mockup interface for tool independent exchange of simulation models. In Christoph Clauß, editor, 8th International Modelica Conference, Linköping Electronic Conference Proceedings, pages 105-114. Linköping University Press, March 2011.

[52] Modelica Association. Functional Mock-up Interface for Model Exchange and CoSimulation, 2021. [Online] Available: http://fmi-standard.org/.

[53] Modelica ${ }^{\circledR}$ - A Unified Object-Oriented Language for Systems ModelingLanguage Specification Version 3.4. Available: https://specification.modelica.org/v3.4/MLS.html.

[54] Modelica tools — modelica association. Available: https://www.modelica.org/tools. 
[55] Hilding Elmqvist. A Structured Model Language for Large Continuous Systems. PhD Thesis, Department of Automatic Control, Lund Institute of Technology (LTH), 1978. ISSN: 03465500 Series: PhD Thesis TFRT-1015.

[56] A Haumer, C Kral, JV Gragg, and H Kapeller. Quasi-Static Modeling and Simulation of Electrical Circuits using Complex Phasors. Bielefeld, March 2008.

[57] Rüdiger Franke and Hansjürg Wiesmann. Flexible modeling of electrical power systems the Modelica PowerSystems library. pages 515-522, March 2014.

[58] Marco Bonvini, Michael Wetter, and TS Noudui. A Modelica package for building-toelectrical grid integration. In Proc. of Fifth German-Austrian IBPSA Conference, volume 1, pages 6-13, 2014.

[59] Daniel L. Gerber, Vagelis Vossos, Wei Feng, Chris Marnay, Bruce Nordman, and Richard Brown. A simulation-based efficiency comparison of $\mathrm{AC}$ and DC power distribution networks in commercial buildings. Applied Energy, 210:1167-1187, 2018.

[60] Achim Zielesny. From curve fitting to machine learning an illustrative guide to scientific data analysis and computational intelligence. Intelligent systems reference library, v. 18. Springer.

[61] K.-L. Du. Search and optimization by metaheuristics: techniques and algorithms inspired by nature. Springer eBook collection. Birkhäuser.

[62] A. Othee, J. Cale, A. Santos, S. Frank, D. Zimmerle, O. Ghatpande, G. Duggan, D. Gerber. Modeling toolkit for comparing ac vs. dc electrical distribution efficiency in buildings. submitted for publication, 2021.

[63] EnergyPlus. Available: https://energyplus.net/. 
[64] S. Frank, B. Ball, D. Gerber, K. Cu, A. Othee, J. Shackelford, R. Brown, and J. Cale. A new co-simulation capability for detailed electrical and whole building energy performance. submitted for publication, 2021. 


\section{Appendix A}

\section{Nonlinear load equations}

Defining power flow equation for the fundamental harmonic and writing it in terms of injected current,

$$
\begin{aligned}
\widetilde{S}_{\mathrm{in}, 4,1} & =\widetilde{V}_{4,1,1} \widetilde{I}_{4,1,1}^{*}=\widetilde{V}_{4,1,1} \cdot g_{4}(1, \cdots) \\
\text { or } g_{4}(1,, \cdots) & =\frac{\widetilde{S}_{\mathrm{in}, 4,1}^{*}}{\widetilde{I}_{4,1,1}^{*}}
\end{aligned}
$$

where $\widetilde{S}_{\mathrm{in}, 4,1}$ is the input apparent power at $h=1$ and similarly, $\widetilde{V}_{4,1,1}$ and $\widetilde{I}_{4,1,1}$ are the voltage and current for device 4 . In the above equation $\widetilde{V}_{4,1, h 1}$ is an unknown state variable. Besides the node voltage, the other unknowns are $\left|\widetilde{S}_{\mathrm{in}, 4,1}\right|$ and $\angle \widetilde{S}_{\mathrm{in}, 4,1}$. The angle can be determined using the converter phase angle model as described below.

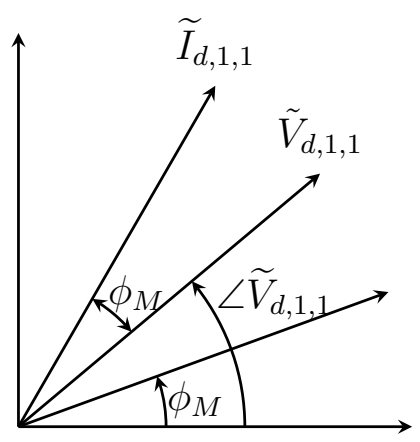

Figure A.1: Phase shift in nonlinear load voltage and current phasor angle.

A load connected to a voltage source via a line impedance will result in phase shift at the load input port. For a harmonic nonlinear load based on current inject spectra model, the phase shift in the voltage would result in the phase shift for the current injection spectra. Figure A.1 shows the voltage phasor angle for a converter with no phase shift (bottom of the figure) and the corresponding phase angle of the current. A line impedance would result in a phase shift in the 
voltage. This phase shift in the fundamental harmonic voltage phasor $\widetilde{V}_{4,1, h}$ is an unknown state variable and is determined by solving the nonlinear system of equations. Using the fact that the phase shift in the current is always relative to the voltage, one can therefore write the new current angle as, $\angle \widetilde{I}_{4,1, h}=\angle \widetilde{V}_{4,1, h}+\phi_{M}$, where $\phi_{M}$ is the phase angle from the device specific behavior model for the nonlinear load at the fundamental harmonic frequency $h=1\left(\phi_{M}=g_{\angle \cdot, d}\left(1, \gamma_{\angle \text {. }}\right)\right)$. Therefore, the phase angle for the apparent power can be written as:

$$
\begin{aligned}
\angle \widetilde{S}_{\mathrm{in}, 4,1} & =\angle \widetilde{V}_{4,1, h}-\angle \widetilde{I}_{4,1, h} \\
& \left.=\angle \widetilde{V}_{4,1, h}-\left(\angle \widetilde{V}_{4,1, h}+\phi_{M}\right)\right) \\
& =-\phi_{M}
\end{aligned}
$$

The other unknown is $\left\|\widetilde{S}_{\text {in, } 4,1}\right\|$ that can obtained from the real power at the fundamental harmonic, $P_{\mathrm{in}, 4,1}=\left\|\widetilde{S}_{\mathrm{in}, 4,1}\right\| \cos \left(\angle \widetilde{S}_{\mathrm{in}, 4,1}\right)$.

Using the notation, $r \operatorname{cis}(\theta)$ as a shorthand for, $r \operatorname{cis}(\theta)=r(\cos (\theta)+i \sin (\theta))$, where $r$ is the magnitude of the complex number and $\theta$ is the angle (polar notation). The device-specific behavioral function can therefore be written as:

$$
g_{4}\left(h, P_{\mathrm{in}, 4,1}, \widetilde{V}_{4,1, h}, \gamma_{4}\right)= \begin{cases}\frac{P_{\mathrm{in}, 4,1}}{\left\|\widetilde{V}_{4,1, h}\right\| \cos \left(\phi_{M}\right)}\left(\operatorname{cis}\left(\angle \widetilde{V}_{4,1, h}+\phi_{M}\right)\right), & h=1 \\ g_{\|\cdot\|, 4}\left(h, P_{\mathrm{in}, 4,1}, \gamma_{\|\cdot\|}\right)\left(\operatorname{cis}\left(g_{\angle \cdot, 4}\left(h, P_{\mathrm{in}, 4,1}, \gamma_{L \cdot}\right)+h \angle \widetilde{I}_{4,1,1}\right)\right), & h>1\end{cases}
$$

Notice the phase angle adjustment in injected converter harmonic currents for higher harmonics $(h>1)$. 


\section{Appendix B}

\section{Code}

\section{B.1 MATLAB code - Example Circuit}

The script implements a harmonic power flow solution for the example nonlinear system in chapter 5 . The script initializes the system by setting start values for the state variables. Network solution is computed using iterative Newton-Raphson root finding algorithm. System Jacobian is obtained numerically, implemented in function numJacobian. System equations are implemented in function handle $f$.

Supporting functions getIndices.m (auxiliary function for state variable indexing) and numJacobian.m are not included and will be provided on request.

\section{B.1.1 Main script}

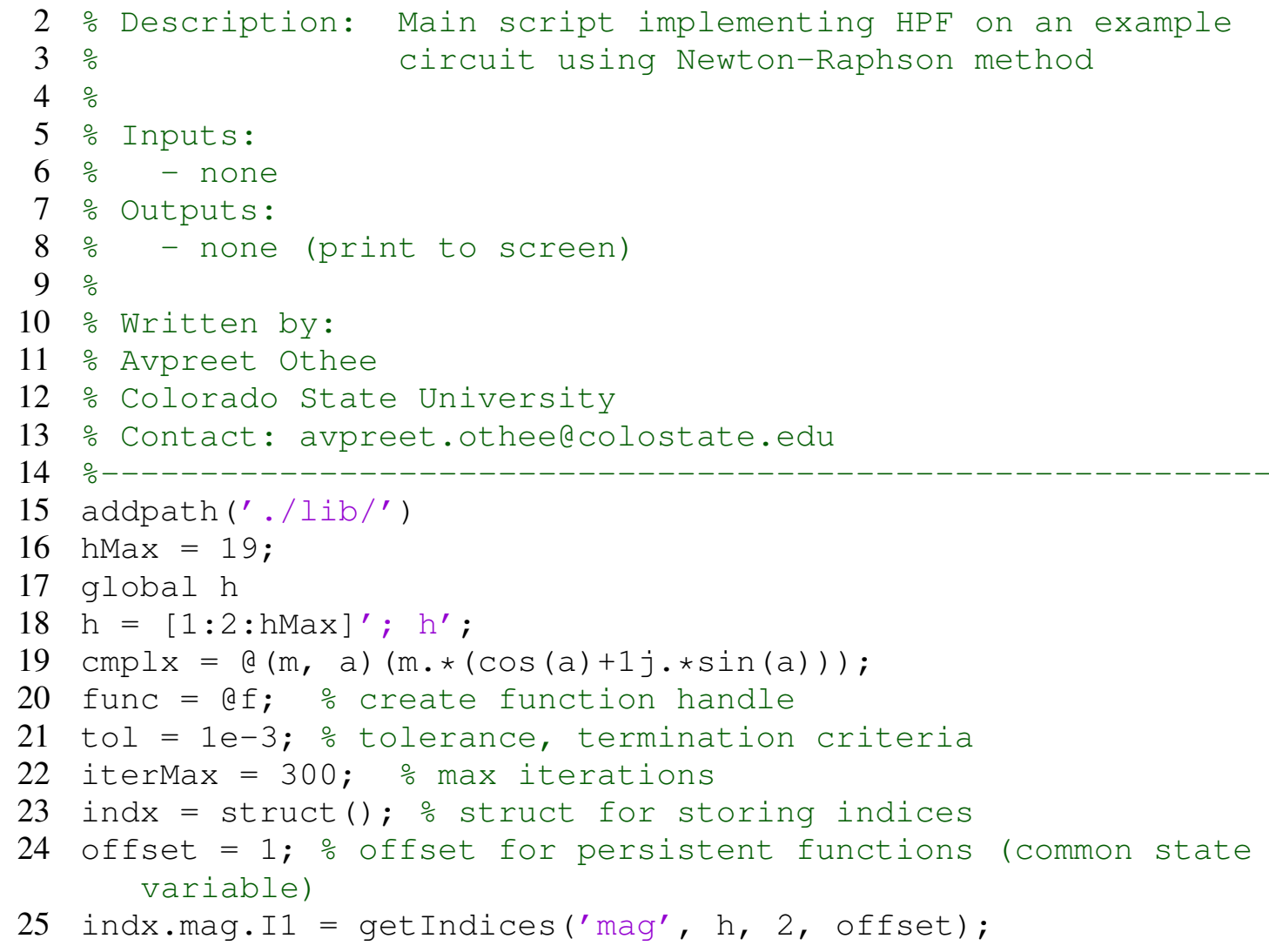




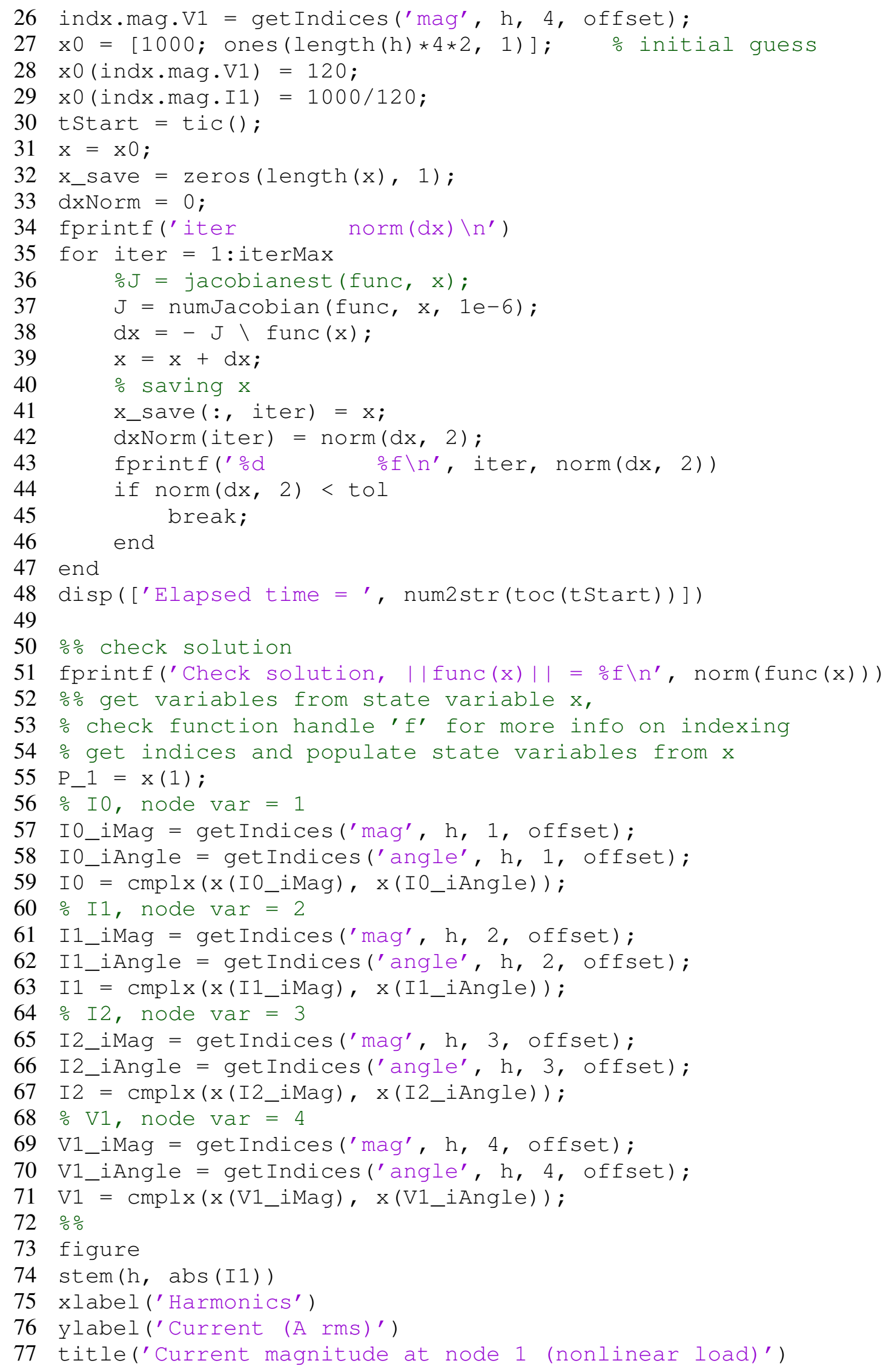


78 figure

79 stem(h, abs (V1))

80 xlabel ('Harmonics')

81 ylabel ('Voltage (V rms)')

82 title('Voltage magnitude at node 1 (nonlinear load)')

\section{B.1.2 Function handle containing system equations}

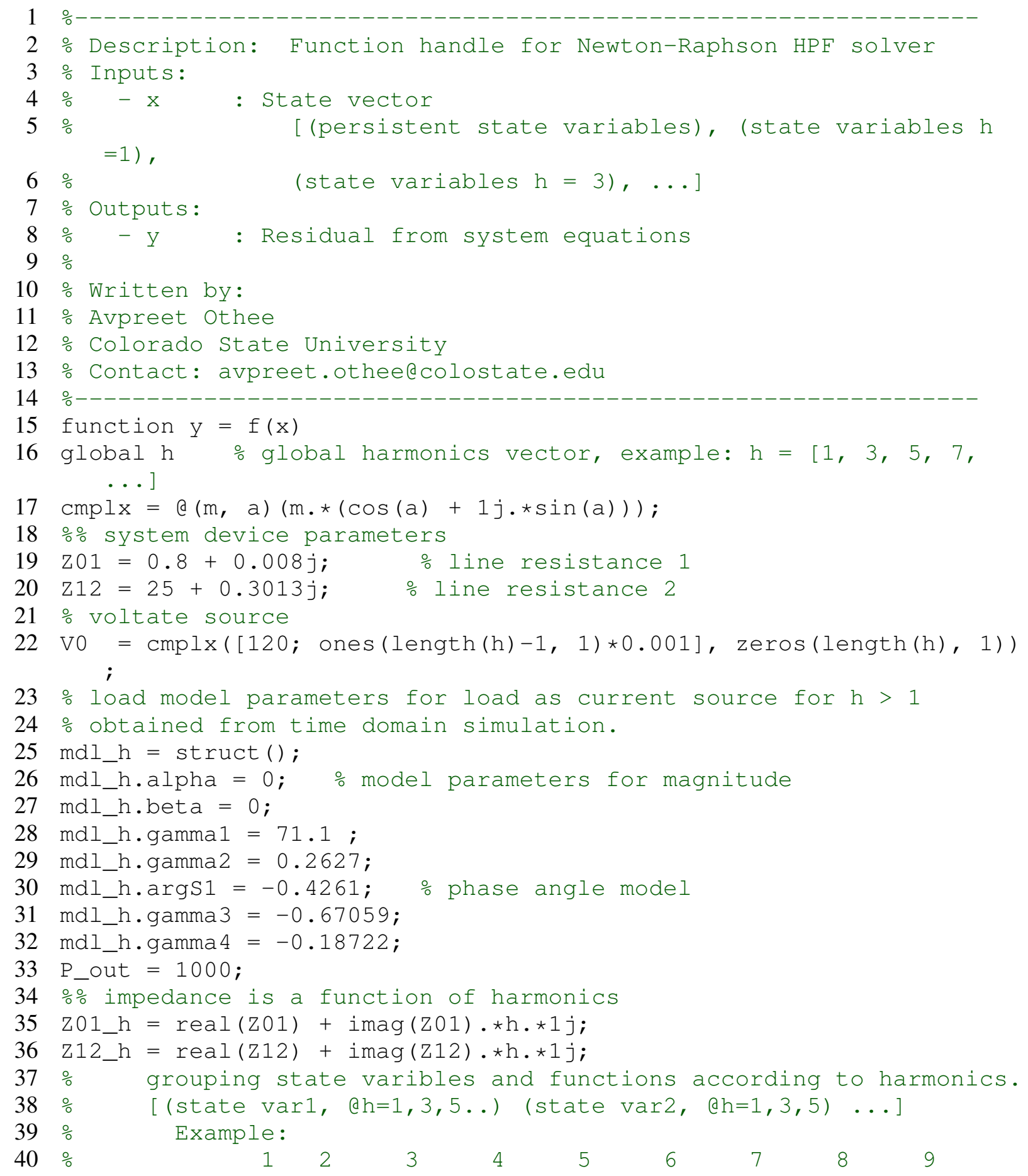




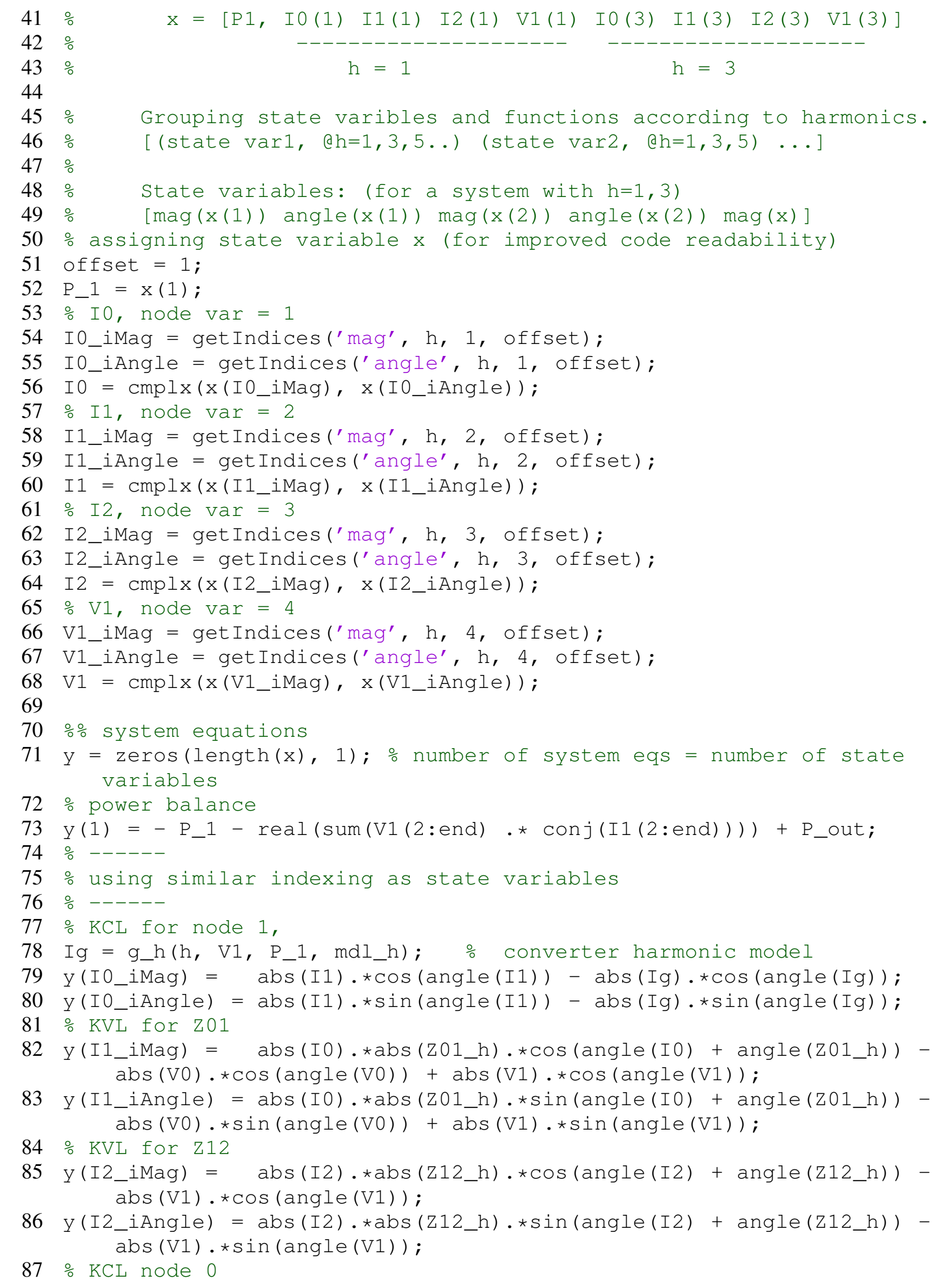


$88 \mathrm{y}(\mathrm{V} 1$ _iMag $)=\operatorname{abs}(\mathrm{I} 0) . \star \cos (\operatorname{angle}(\mathrm{I} 0))-\operatorname{abs}(\mathrm{I} 1) . \star \cos (\operatorname{angle}(\mathrm{I} 1))-$ abs (I2) **cos (angle (I2));

89 y $(V 1$ ViAngle $)=\operatorname{abs}($ IO $) . * \sin (\operatorname{angle}(\mathrm{I} 0))-\operatorname{abs}(\mathrm{I} 1) . * \sin (\operatorname{angle}(\mathrm{I} 1))-$ abs (I2) .*sin (angle (I2));

90 end

\section{B.1.3 Nonlinear load model}

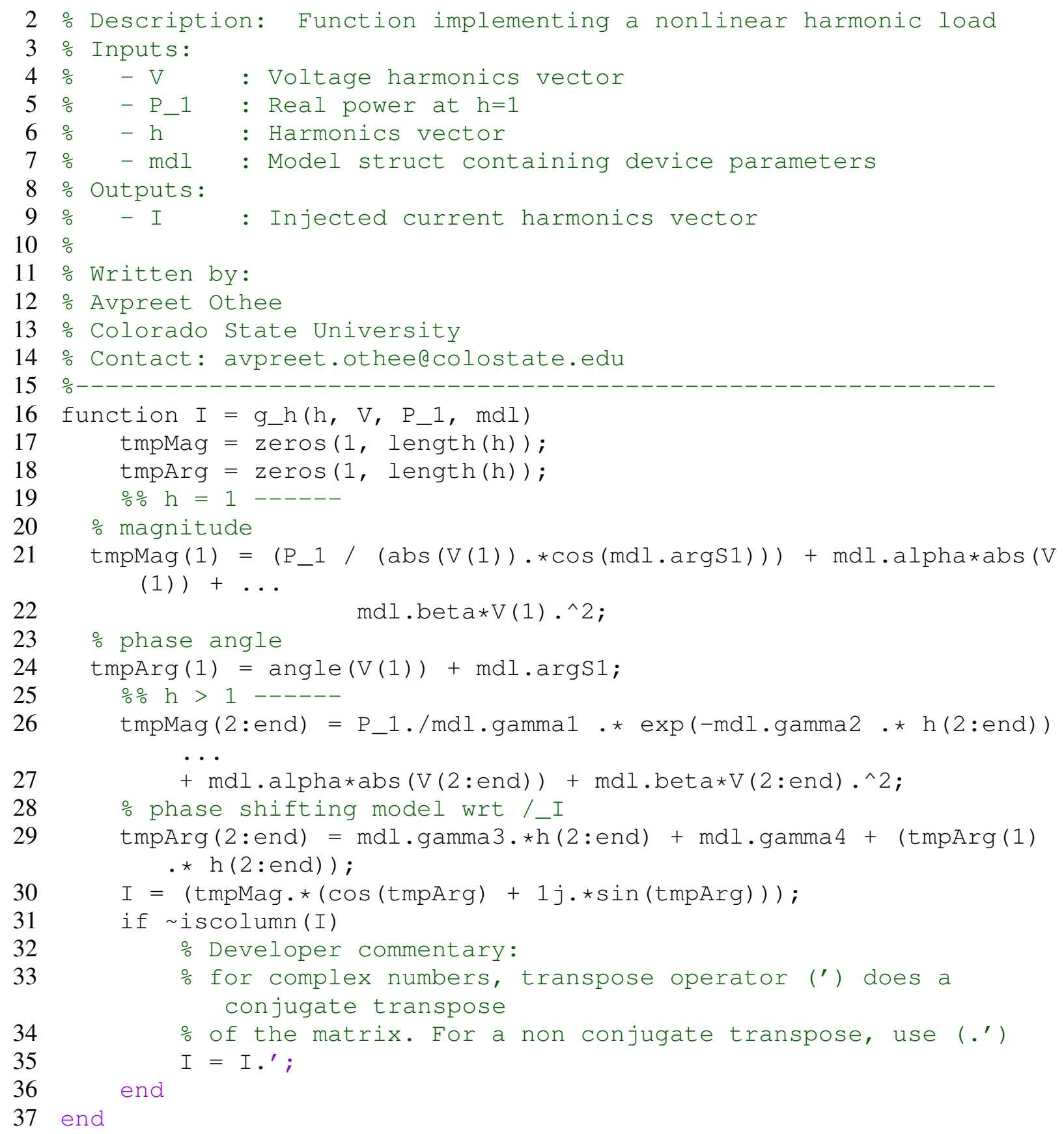




\title{
B.2 Modelica Code - Example Circuit
}

\author{
B.2.1 Simulation model \\ model ExampleSystem \\ import Modelica.ComplexMath.j; \\ inner HPF. SystemDef systemDef(hrms $=\{i$ for $i$ in $1: 2: 25\})$; \\ HarmonicLoad load (start_v_re $=\operatorname{cat}(1,\{120\},\{0.001$ for $i$ in 1 : \\ systemDef.numHrm - 1\})); \\ HPF.SinglePhase.Components. Impedance $\mathrm{Zl}(\mathrm{z}=0.8+0.008 * j)$; \\ HPF.SinglePhase.Components. Impedance $\mathrm{Z} 2(\mathrm{z}=25+0.3013 * j$, \\ start_v_re $=\operatorname{cat}(1,\{120\},\{0.0$ for $i$ in 1 :systemDef.numHrm - \\ 1\})); \\ 7 HPF.SinglePhase.Components.Ground ground; \\ 8 HPF.SinglePhase.Sources.VoltageSource VO (vArg $=\{0$ for $i$ in 1 : \\ systemDef.numHrm $\}, \operatorname{vMag}=\operatorname{cat}(1,\{120\},\{0$ for $i$ in 2 : \\ equation \\ systemDef.numHrm\}));

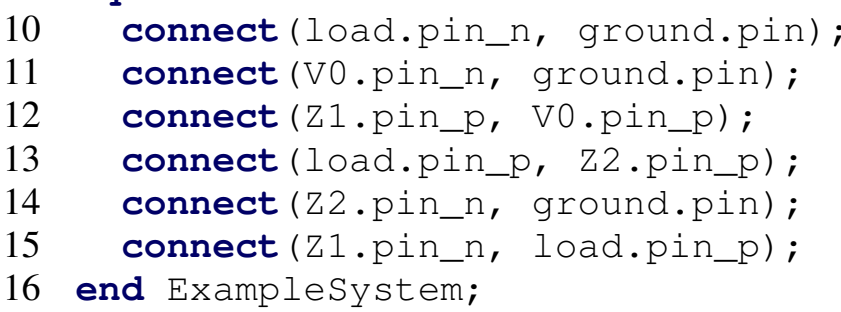

\section{B.2.2 Nonlinear load}

model HarmonicLoad "Harmonic source"

extends HPF.SinglePhase.Interface.TwoPinBase;

parameter Real $\mathrm{P}=1000$ "Real power drawn by DC side";

parameter Real alpha $=3 e-2$;

parameter Real beta $=3 e-4$;

parameter Real gamma $=0.2627$;

parameter Real nu $=71.1$;

parameter Real $m=-0.67059$ "Phase model coef $1 "$;

parameter Real $\mathrm{c}=-0.18722$ "Phase model coef 2";

10 parameter Real argS1 $=-0.4261$ "Phase angle for fundamental

apparent power";

11 Real P1 (start $=$ P, fixed = false) "Real power at fundamental";

12 Real tmp_Ph[systemDef.numHrm - 1] "tmp variable for sum of power $\mathrm{h}>1 "$;

13 Real tmpArg "phase angle for I \& $\mathrm{h}=1$ ";

14 Real vMag[systemDef.numHrm] = Modelica.ComplexMath.'abs' (v[:]);

15 Real iMag[systemDef.numHrm] = Modelica.ComplexMath.'abs' (i[:]);

16 Real iArg[systemDef.numHrm] = Modelica.ComplexMath.arg(i[:]);

17 Real vArg[systemDef.numHrm] = Modelica.ComplexMath.arg(v[:]);

18 equation

19 tmp_Ph[:] = v[2:systemDef.numHrm].re .* i[2:systemDef.numHrm].re .+ $v[2$ :systemDef.numHrm].im .* i[2:systemDef.numHrm].im; 


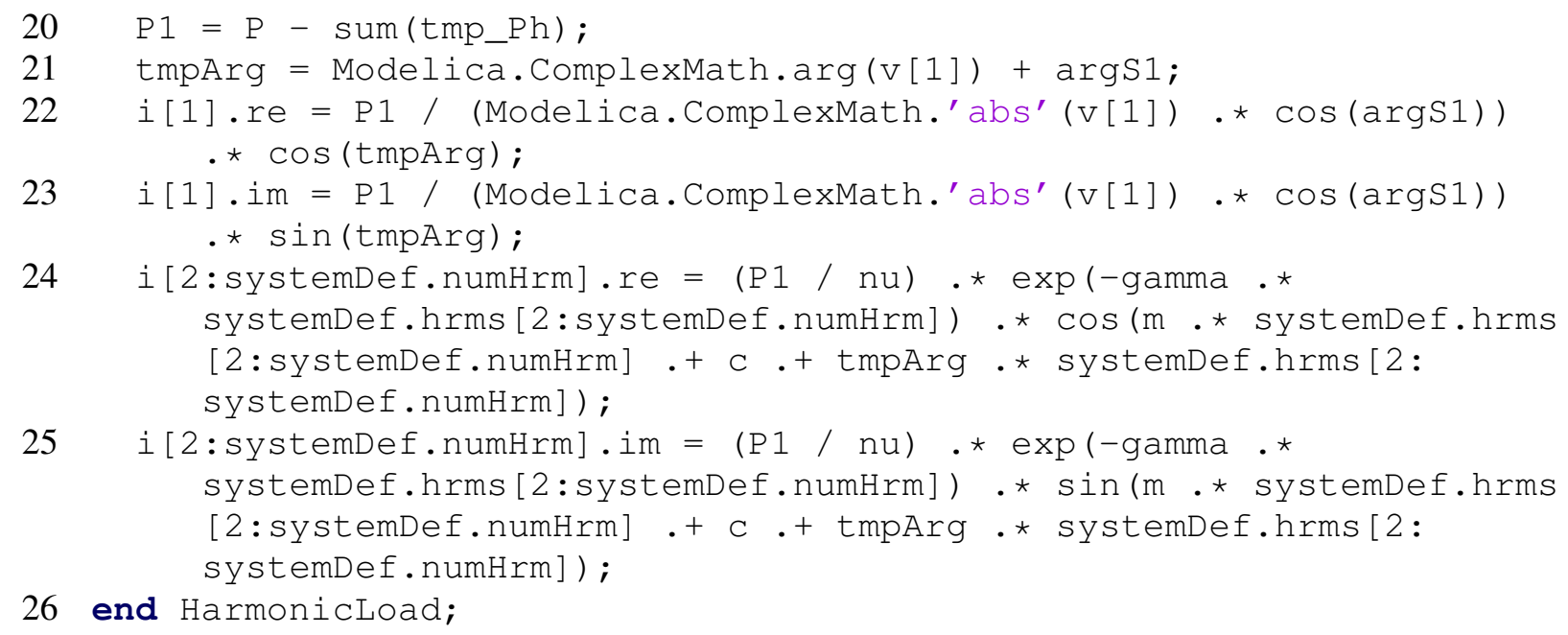

\section{B.3 BEEAM Library Source Code}

As of writing of this dissertation, the source of the library has been released under a permissive open source license (BSD-3). The source code can be accessed at: https://github.com/NREL/ BEEAM 


\section{Appendix C}

\section{Simulation Results}

For all scenarios, the average values of the measured device powers were used as the input to the associated power flow model. Modeled values for input power $P_{\mathrm{In}}$, transformer secondary power $P_{\text {Sec }}$, transformer loss $P_{\text {LossTran }}$, converter loss $P_{\text {LossConv }}$, total system loss $P_{\text {Loss }}$, and system efficiency $\eta$ were extracted from the simulation results.

Tables C.1-C.3 compare the measured and modeled values of these quantities. Figures C.1C.3 show the predicted and measured total system input power for all scenarios. Figures C.4-C.6 show the predicted and measured transformer power at the secondary. Figures C.7-C.9 show the predicted and measured transformer losses. And finally, Figures C.10-C.12 show the predicted and measured converter losses. 
Table C.1: Measured and modeled values for system input power and transformer secondary power.

\begin{tabular}{|c|r|r|r|r|}
\hline & System Input Power $P_{\text {In }}$ & \multicolumn{2}{|c|}{ Transformer Secondary Power $P_{\text {Sec }}$} \\
\hline Scenario & Measured & Modeled & Measured & Modeled \\
\hline 1.1 & $101.8(20) \mathrm{W}$ & $99.2 \mathrm{~W}$ & $50.3(17) \mathrm{W}$ & $51.3 \mathrm{~W}$ \\
\hline 1.2 & $460.7(24) \mathrm{W}$ & $449.2 \mathrm{~W}$ & $403.4(22) \mathrm{W}$ & $394.8 \mathrm{~W}$ \\
\hline 1.3 & $1254.9(32) \mathrm{W}$ & $1243.7 \mathrm{~W}$ & $1172.2(26) \mathrm{W}$ & $1161.4 \mathrm{~W}$ \\
\hline 1.4 & $1870.9(30) \mathrm{W}$ & $1887.9 \mathrm{~W}$ & $1750.2(28) \mathrm{W}$ & $1749.9 \mathrm{~W}$ \\
\hline 2.1 & $101.6(20) \mathrm{W}$ & $99.2 \mathrm{~W}$ & $50.3(17) \mathrm{W}$ & $51.3 \mathrm{~W}$ \\
\hline 2.2 & $1772.1(28) \mathrm{W}$ & $1796.9 \mathrm{~W}$ & $1651.1(38) \mathrm{W}$ & $1654.9 \mathrm{~W}$ \\
\hline 2.3 & $1839.2(30) \mathrm{W}$ & $1861.4 \mathrm{~W}$ & $1709.4(27) \mathrm{W}$ & $1711 \mathrm{~W}$ \\
\hline 2.4 & $1583.9(30) \mathrm{W}$ & $1599.2 \mathrm{~W}$ & $1482.3(33) \mathrm{W}$ & $1483.9 \mathrm{~W}$ \\
\hline 3.1 & $56.8(20) \mathrm{W}$ & $53.4 \mathrm{~W}$ & $5.3(16) \mathrm{W}$ & $5.3 \mathrm{~W}$ \\
\hline 3.2 & $415.3(25) \mathrm{W}$ & $400.4 \mathrm{~W}$ & $357.6(22) \mathrm{W}$ & $346.8 \mathrm{~W}$ \\
\hline 3.3 & $985.3(15) \mathrm{W}$ & $966.2 \mathrm{~W}$ & $919.3(13) \mathrm{W}$ & $901.9 \mathrm{~W}$ \\
\hline 3.4 & $1613.2(36) \mathrm{W}$ & $1598.8 \mathrm{~W}$ & $1529.2(36) \mathrm{W}$ & $1511.1 \mathrm{~W}$ \\
\hline 4.1 & $56.8(20) \mathrm{W}$ & $53.4 \mathrm{~W}$ & $5.3(16) \mathrm{W}$ & $5.3 \mathrm{~W}$ \\
\hline 4.2 & $1616.7(29) \mathrm{W}$ & $1602.4 \mathrm{~W}$ & $1522.8(27) \mathrm{W}$ & $1503.6 \mathrm{~W}$ \\
\hline 4.3 & $1612.9(19) \mathrm{W}$ & $1596.9 \mathrm{~W}$ & $1523.4(18) \mathrm{W}$ & $1505.6 \mathrm{~W}$ \\
\hline 4.4 & $1407.0(31) \mathrm{W}$ & $1391.1 \mathrm{~W}$ & $1322.5(36) \mathrm{W}$ & $1303.9 \mathrm{~W}$ \\
\hline 5.1 & $72.4(20) \mathrm{W}$ & $67.4 \mathrm{~W}$ & $19.8(7) \mathrm{W}$ & $19.5 \mathrm{~W}$ \\
\hline 5.2 & $294.2(15) \mathrm{W}$ & $283.7 \mathrm{~W}$ & $239.7(19) \mathrm{W}$ & $233.3 \mathrm{~W}$ \\
\hline 5.3 & $693.0(16) \mathrm{W}$ & $690.1 \mathrm{~W}$ & $627.5(14) \mathrm{W}$ & $623.6 \mathrm{~W}$ \\
\hline 5.4 & $1081.1(40) \mathrm{W}$ & $1095.9 \mathrm{~W}$ & $992.9(53) \mathrm{W}$ & $998.5 \mathrm{~W}$ \\
\hline 6.1 & $71.9(20) \mathrm{W}$ & $68.3 \mathrm{~W}$ & $19.4(8) \mathrm{W}$ & $20.5 \mathrm{~W}$ \\
\hline 6.2 & $290.5(33) \mathrm{W}$ & $278.3 \mathrm{~W}$ & $236.6(31) \mathrm{W}$ & $228.7 \mathrm{~W}$ \\
\hline 6.3 & $680.7(14) \mathrm{W}$ & $674.1 \mathrm{~W}$ & $619.2(10) \mathrm{W}$ & $613.8 \mathrm{~W}$ \\
\hline 6.4 & $1051.7(32) \mathrm{W}$ & $1055.6 \mathrm{~W}$ & $975.2(48) \mathrm{W}$ & $976.4 \mathrm{~W}$ \\
\hline
\end{tabular}


Table C.2: Measured and modeled values for transformer and converter losses.

\begin{tabular}{|c|r|r|r|r|}
\hline & Transformer Loss $P_{\text {LossTran }}$ & \multicolumn{2}{|c|}{ Converter Loss $P_{\text {LossConv }}$} \\
\hline Scenario & Measured & Modeled & Measured & Modeled \\
\hline 1.1 & $51.5(26) \mathrm{W}$ & $47.9 \mathrm{~W}$ & $50.3(17) \mathrm{W}$ & $51.3 \mathrm{~W}$ \\
\hline 1.2 & $57.3(33) \mathrm{W}$ & $54.4 \mathrm{~W}$ & $104.2(31) \mathrm{W}$ & $95.4 \mathrm{~W}$ \\
\hline 1.3 & $82.7(42) \mathrm{W}$ & $82.3 \mathrm{~W}$ & $175.0(32) \mathrm{W}$ & $165.2 \mathrm{~W}$ \\
\hline 1.4 & $120.6(41) \mathrm{W}$ & $138 \mathrm{~W}$ & $220.8(38) \mathrm{W}$ & $220.8 \mathrm{~W}$ \\
\hline 2.1 & $51.3(26) \mathrm{W}$ & $47.9 \mathrm{~W}$ & $50.3(17) \mathrm{W}$ & $51.3 \mathrm{~W}$ \\
\hline 2.2 & $121.0(47) \mathrm{W}$ & $142 \mathrm{~W}$ & $215.5(54) \mathrm{W}$ & $219 \mathrm{~W}$ \\
\hline 2.3 & $129.8(40) \mathrm{W}$ & $150.3 \mathrm{~W}$ & $216.3(39) \mathrm{W}$ & $217.7 \mathrm{~W}$ \\
\hline 2.4 & $101.6(45) \mathrm{W}$ & $115.3 \mathrm{~W}$ & $200.3(43) \mathrm{W}$ & $201 \mathrm{~W}$ \\
\hline 3.1 & $51.5(26) \mathrm{W}$ & $48 \mathrm{~W}$ & $5.3(16) \mathrm{W}$ & $5.3 \mathrm{~W}$ \\
\hline 3.2 & $57.7(33) \mathrm{W}$ & $53.7 \mathrm{~W}$ & $60.0(32) \mathrm{W}$ & $49.2 \mathrm{~W}$ \\
\hline 3.3 & $66(2) \mathrm{W}$ & $64.3 \mathrm{~W}$ & $66.2(128) \mathrm{W}$ & $48.8 \mathrm{~W}$ \\
\hline 3.4 & $84(5) \mathrm{W}$ & $87.7 \mathrm{~W}$ & $67.0(269) \mathrm{W}$ & $48.9 \mathrm{~W}$ \\
\hline 4.1 & $51.5(26) \mathrm{W}$ & $48 \mathrm{~W}$ & $5.3(16) \mathrm{W}$ & $5.3 \mathrm{~W}$ \\
\hline 4.2 & $93.9(39) \mathrm{W}$ & $98.8 \mathrm{~W}$ & $68.1(243) \mathrm{W}$ & $48.8 \mathrm{~W}$ \\
\hline 4.3 & $89.6(26) \mathrm{W}$ & $91.3 \mathrm{~W}$ & $66.5(243) \mathrm{W}$ & $48.7 \mathrm{~W}$ \\
\hline 4.4 & $84.6(48) \mathrm{W}$ & $87.3 \mathrm{~W}$ & $67.4(231) \mathrm{W}$ & $48.8 \mathrm{~W}$ \\
\hline 5.1 & $52.6(21) \mathrm{W}$ & $47.9 \mathrm{~W}$ & $19.8(7) \mathrm{W}$ & $19.5 \mathrm{~W}$ \\
\hline 5.2 & $54.5(25) \mathrm{W}$ & $50.4 \mathrm{~W}$ & $41.7(26) \mathrm{W}$ & $35.3 \mathrm{~W}$ \\
\hline 5.3 & $65.5(21) \mathrm{W}$ & $66.6 \mathrm{~W}$ & $75.4(30) \mathrm{W}$ & $71.4 \mathrm{~W}$ \\
\hline 5.4 & $88.3(67) \mathrm{W}$ & $97.4 \mathrm{~W}$ & $108.7(62) \mathrm{W}$ & $114.4 \mathrm{~W}$ \\
\hline 6.1 & $52.5(22) \mathrm{W}$ & $47.8 \mathrm{~W}$ & $19.4(8) \mathrm{W}$ & $20.5 \mathrm{~W}$ \\
\hline 6.2 & $53.9(45) \mathrm{W}$ & $49.5 \mathrm{~W}$ & $40.5(41) \mathrm{W}$ & $32.6 \mathrm{~W}$ \\
\hline 6.3 & $61.5(17) \mathrm{W}$ & $60.3 \mathrm{~W}$ & $66.1(27) \mathrm{W}$ & $93.7 \mathrm{~W}$ \\
\hline 6.4 & $76.5(58) \mathrm{W}$ & $79.1 \mathrm{~W}$ & $92.4(60) \mathrm{W}$ & \\
\hline
\end{tabular}


Table C.3: Measured and modeled values for total system losses and efficiency.

\begin{tabular}{|c|c|c|c|c|}
\hline & \multicolumn{2}{|c|}{ Total System Loss $P_{\text {Loss }}$} & \multicolumn{2}{|c|}{ Efficiency $\eta$} \\
\hline Scenario & Measured & Modeled & Measured & Modeled \\
\hline 1.1 & $101.8(20) \mathrm{W}$ & $99.2 \mathrm{~W}$ & - & - \\
\hline 1.2 & $161.5(33) \mathrm{W}$ & $149.9 \mathrm{~W}$ & $64.9(4) \%$ & $66.6 \%$ \\
\hline 1.3 & $257.7(37) \mathrm{W}$ & $247.5 \mathrm{~W}$ & $79.5(3) \%$ & $80.1 \%$ \\
\hline 1.4 & $341.4(40) \mathrm{W}$ & $358.8 \mathrm{~W}$ & $81.8(2) \%$ & $81 \%$ \\
\hline 2.1 & $101.6(20) \mathrm{W}$ & $99.2 \mathrm{~W}$ & - & - \\
\hline 2.2 & $336.5(48) \mathrm{W}$ & $361 \mathrm{~W}$ & $81.0(3) \%$ & $79.9 \%$ \\
\hline 2.3 & $346.1(41) \mathrm{W}$ & $368.1 \mathrm{~W}$ & $81.2(2) \%$ & $80.2 \%$ \\
\hline 2.4 & $301.9(40) \mathrm{W}$ & $316.3 \mathrm{~W}$ & $80.9(2) \%$ & $80.2 \%$ \\
\hline 3.1 & $56.8(20) \mathrm{W}$ & $53.4 \mathrm{~W}$ & - & - \\
\hline 3.2 & $117.7(34) \mathrm{W}$ & $102.8 \mathrm{~W}$ & $71.7 \% \dagger$ & $74.3 \%$ \\
\hline 3.3 & $132.2(129) \mathrm{W}$ & $113.1 \mathrm{~W}$ & $86.6(13) \%$ & $88.3 \%$ \\
\hline 3.4 & $151.0(269) \mathrm{W}$ & $136.6 \mathrm{~W}$ & $90.6(16) \%$ & $91.5 \%$ \\
\hline 4.1 & $56.8(20) \mathrm{W}$ & $53.4 \mathrm{~W}$ & - & - \\
\hline 4.2 & $161.9(243) \mathrm{W}$ & $147.6 \mathrm{~W}$ & $90.0(15) \%$ & $90.8 \%$ \\
\hline 4.3 & $156.0(243) \mathrm{W}$ & $140 \mathrm{~W}$ & $90.3(15) \%$ & $91.2 \%$ \\
\hline 4.4 & $152.0(231) \mathrm{W}$ & $136.1 \mathrm{~W}$ & $89.2(16) \%$ & $90.2 \%$ \\
\hline 5.1 & $72.4(20) \mathrm{W}$ & $67.4 \mathrm{~W}$ & - & - \\
\hline 5.2 & $96.2(24) \mathrm{W}$ & $85.7 \mathrm{~W}$ & $67.3(1) \%$ & $69.8 \%$ \\
\hline 5.3 & $140.9(31) \mathrm{W}$ & $138 \mathrm{~W}$ & $79.7(5) \%$ & $80 \%$ \\
\hline 5.4 & $197.0(51) W$ & $211.8 \mathrm{~W}$ & $81.8(5) \%$ & $80.7 \%$ \\
\hline 6.1 & $71.9(20) \mathrm{W}$ & $68.3 \mathrm{~W}$ & - & - \\
\hline 6.2 & $94.4(43) \mathrm{W}$ & $82.2 \mathrm{~W}$ & $67.5 \% \dagger$ & $70.5 \%$ \\
\hline 6.3 & 127.6(29)W & $121 \mathrm{~W}$ & $81.3(4) \%$ & $82.1 \%$ \\
\hline 6.4 & $169.0(48) W$ & $172.8 \mathrm{~W}$ & $83.9(6) \%$ & $83.6 \%$ \\
\hline
\end{tabular}

$\dagger$ Uncertainty value could not be calculated due to large estimate of covariance. 


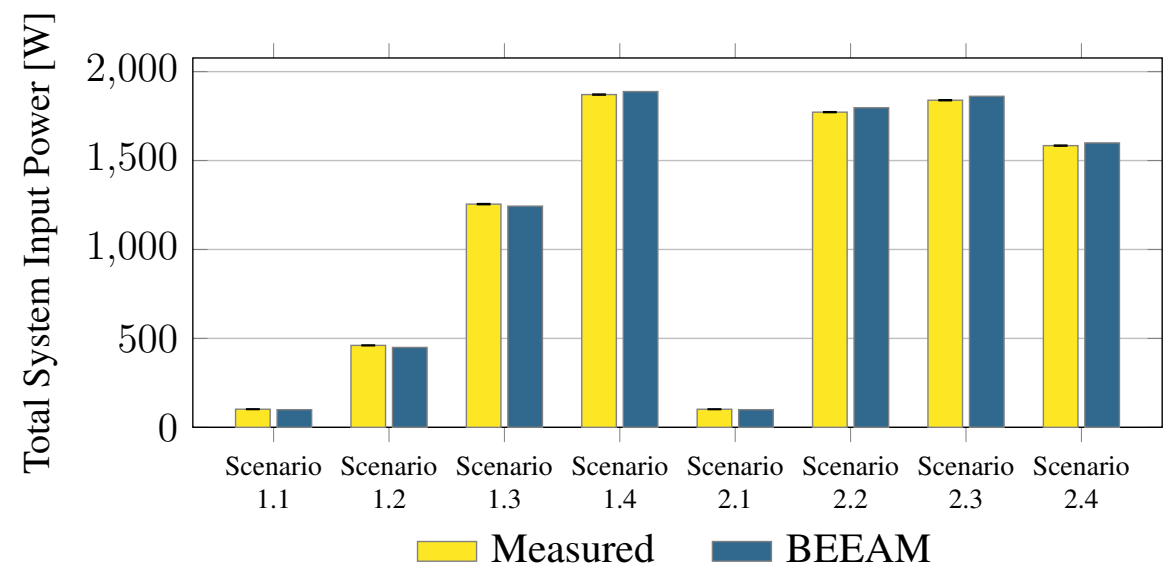

Figure C.1: Predicted versus measured total system input power, Scenario 1-2.

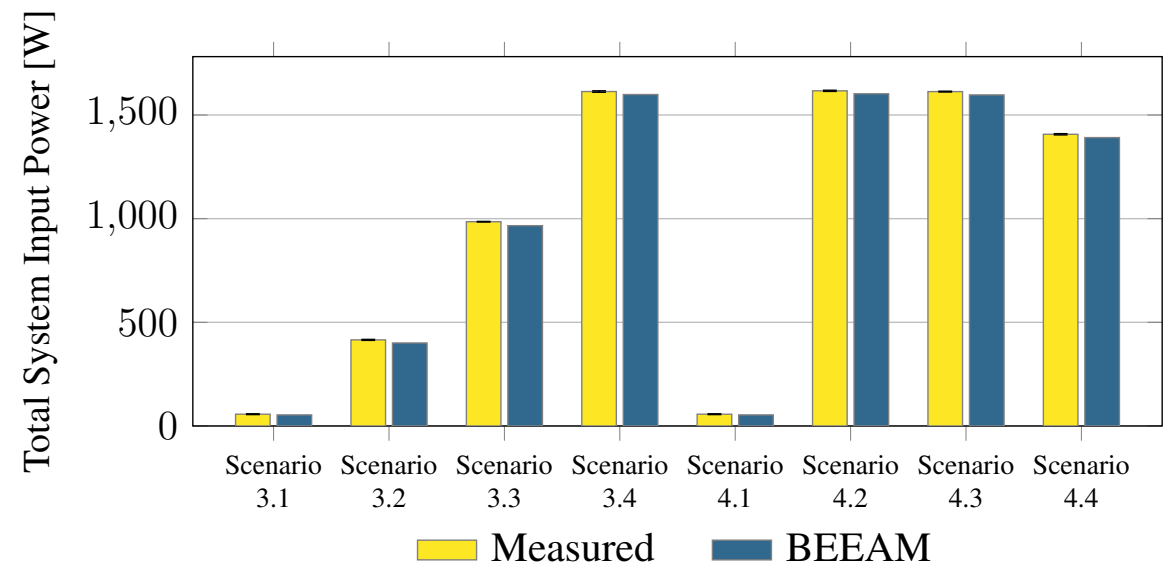

Figure C.2: Predicted versus measured total system input power, Scenario 3-4. 


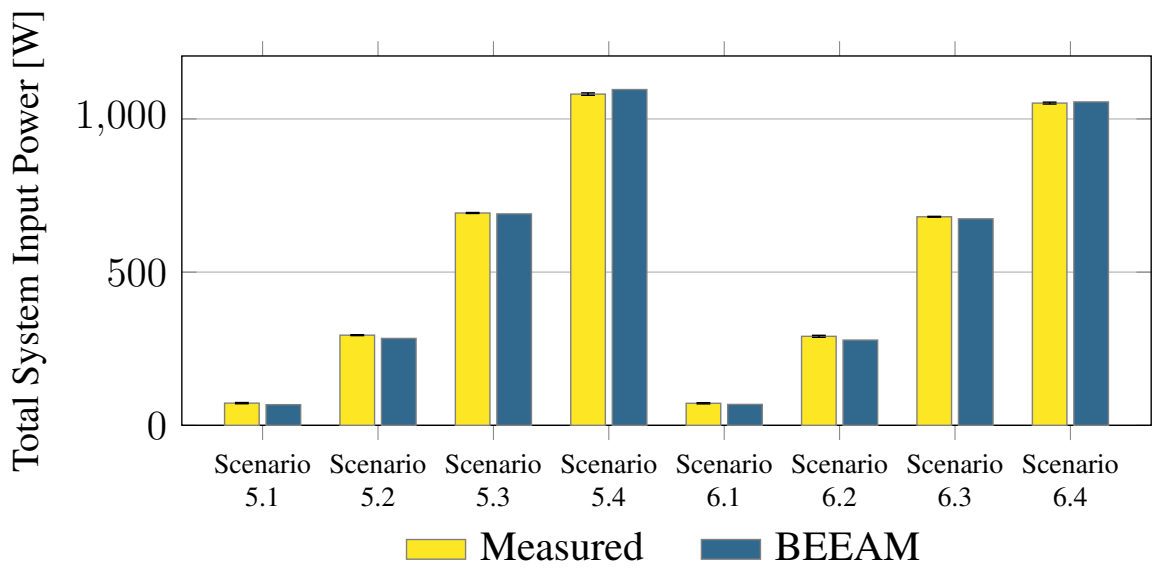

Figure C.3: Predicted versus measured total system input power, Scenario 5-6.

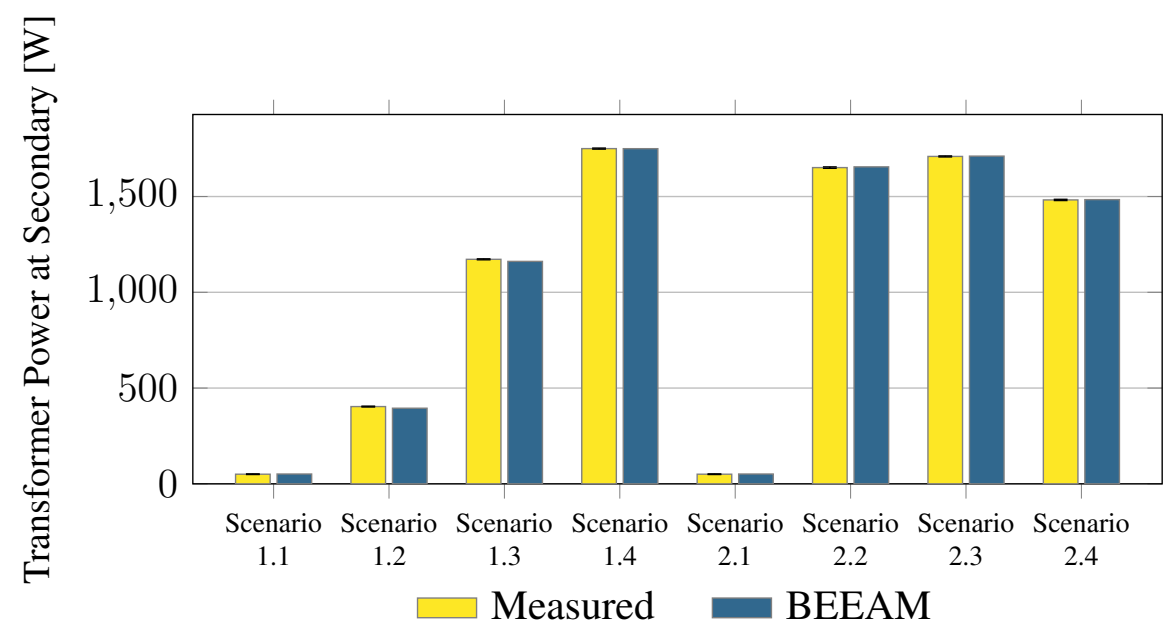

Figure C.4: Predicted versus measured transformer power at secondary, Scenario 1-2.

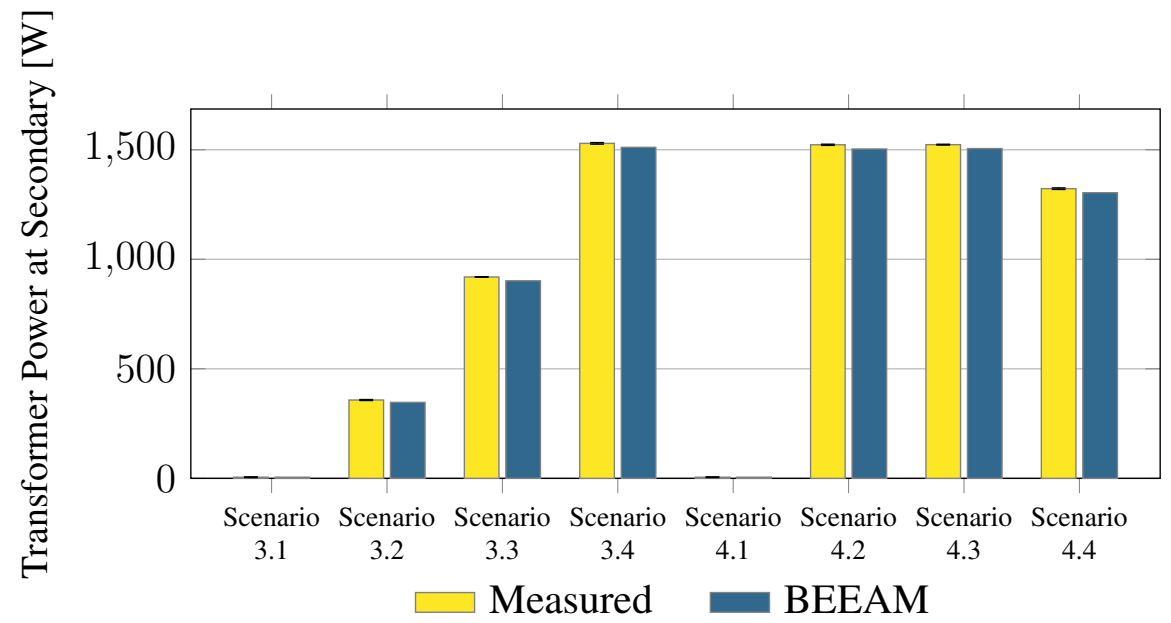

Figure C.5: Predicted versus measured transformer power at secondary, Scenario 3-4. 


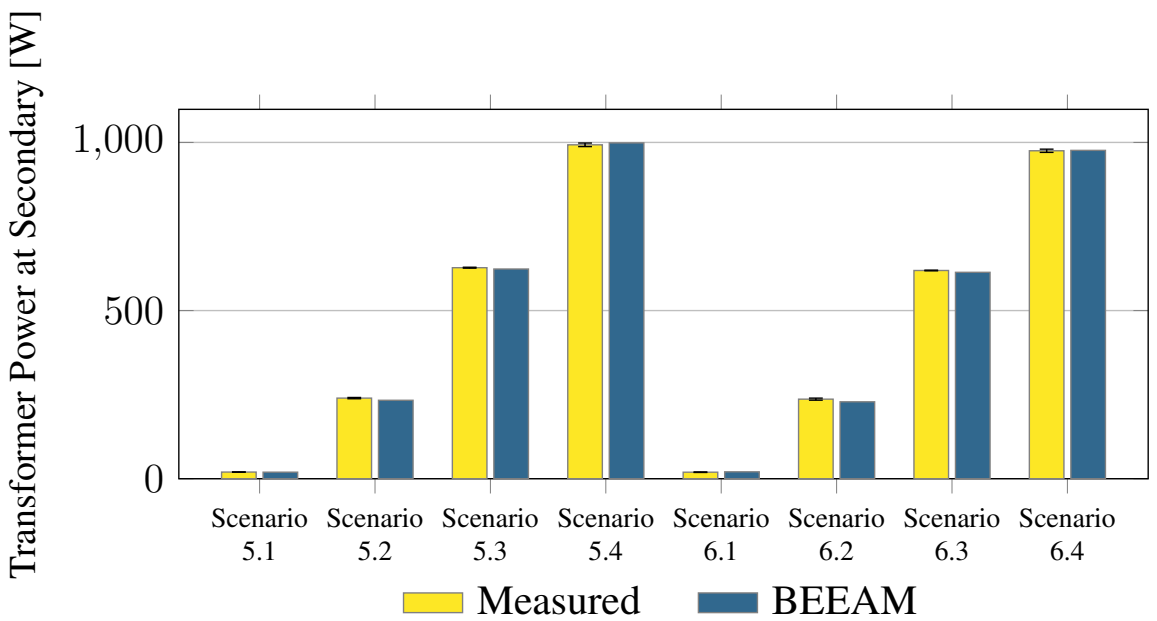

Figure C.6: Predicted versus measured transformer power at secondary, Scenario 5-6.

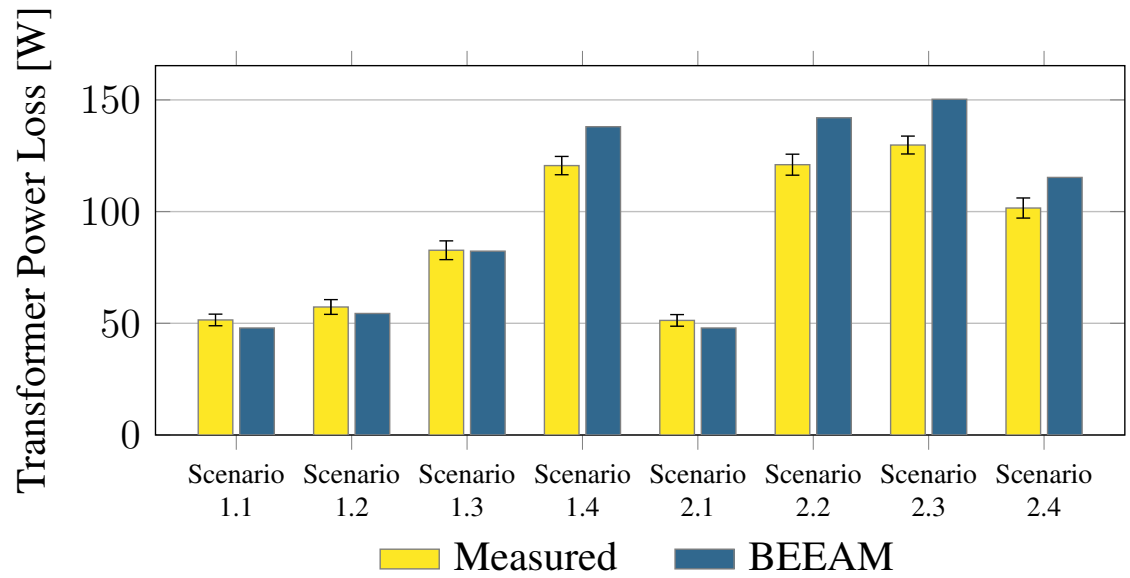

Figure C.7: Predicted versus measured transformer loss, Scenario 1-2.

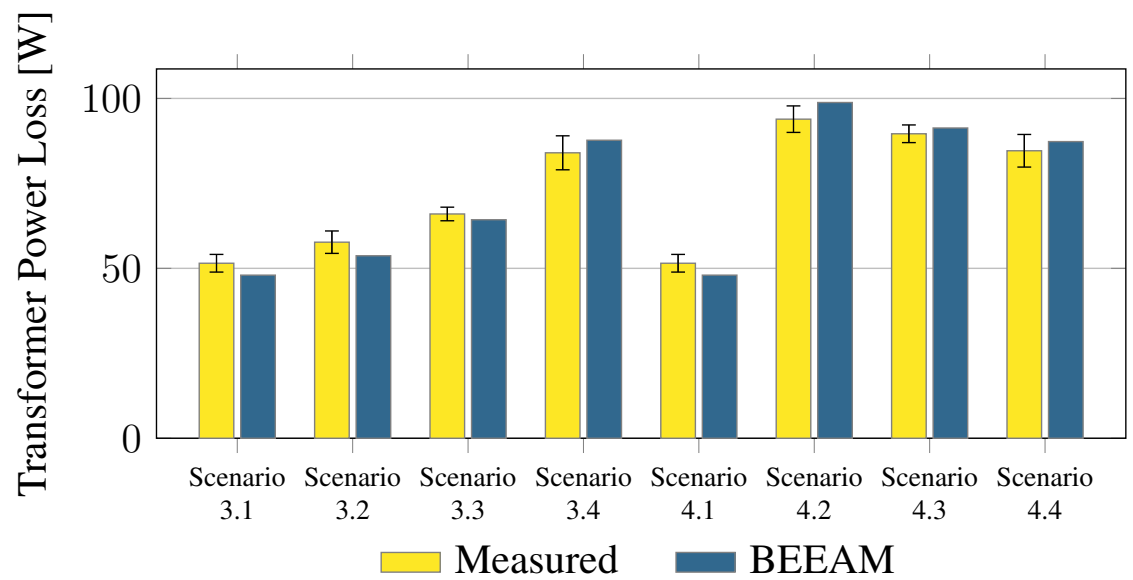

Figure C.8: Predicted versus measured transformer loss, Scenario 3-4. 


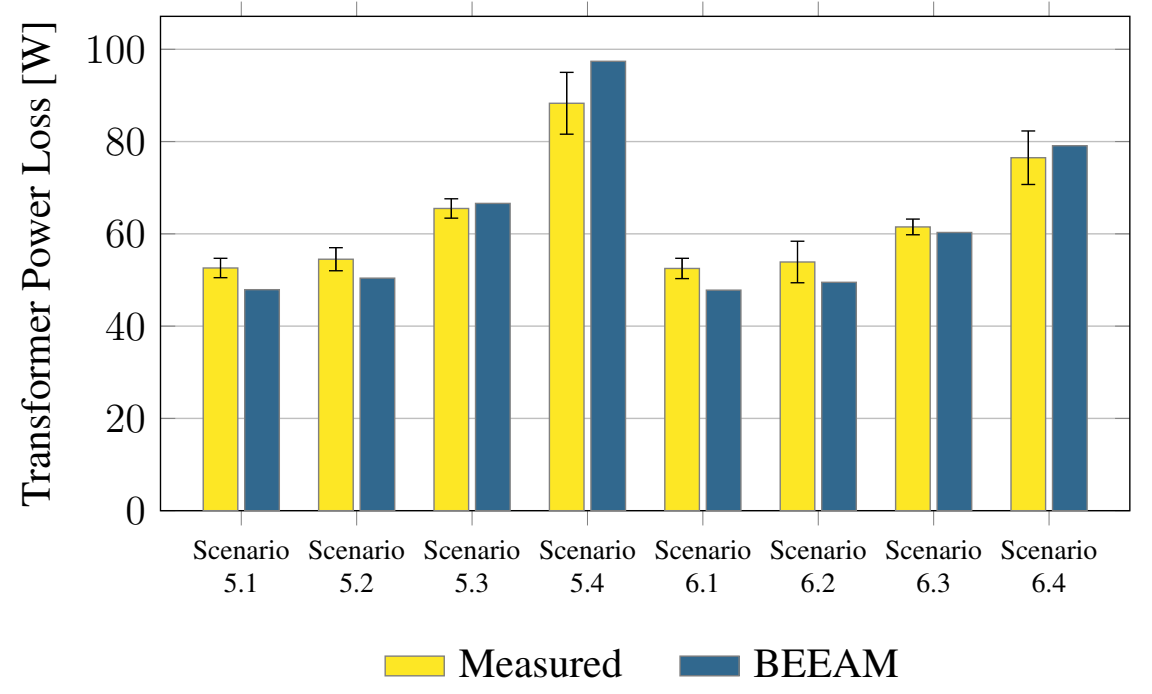

Figure C.9: Predicted versus measured transformer loss, Scenario 5-6.

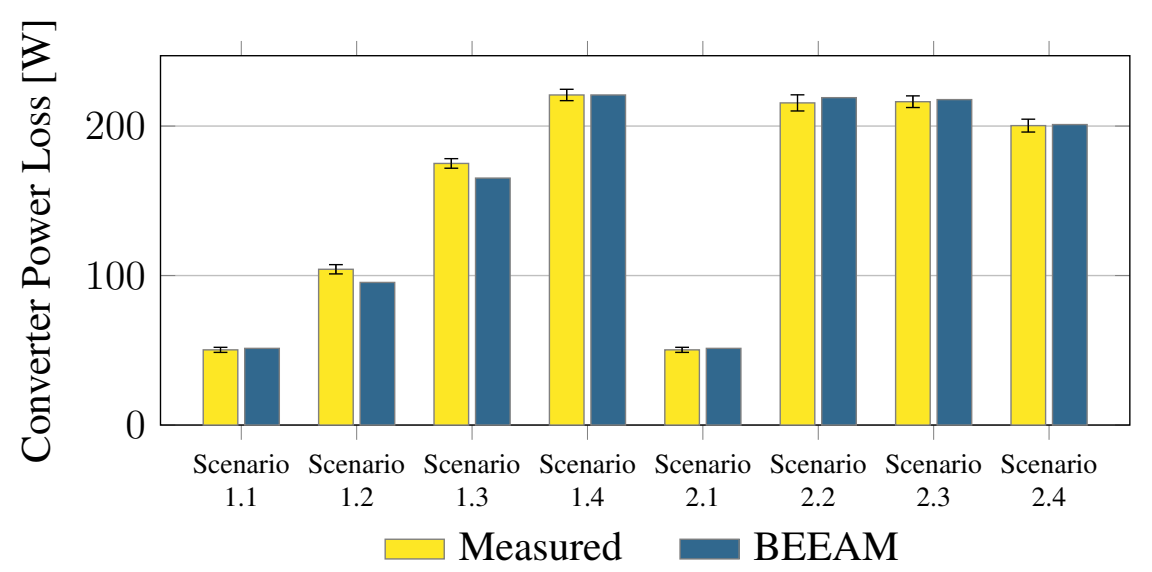

Figure C.10: Predicted versus measured converter loss, Scenario 1-2.

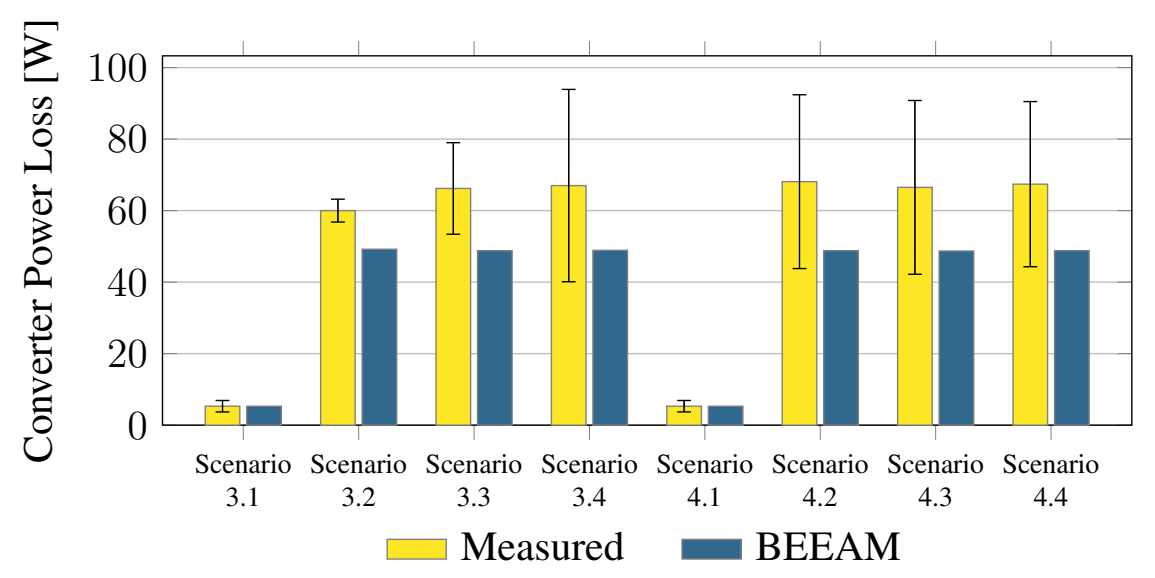

Figure C.11: Predicted versus measured converter loss, Scenario 3-4. 


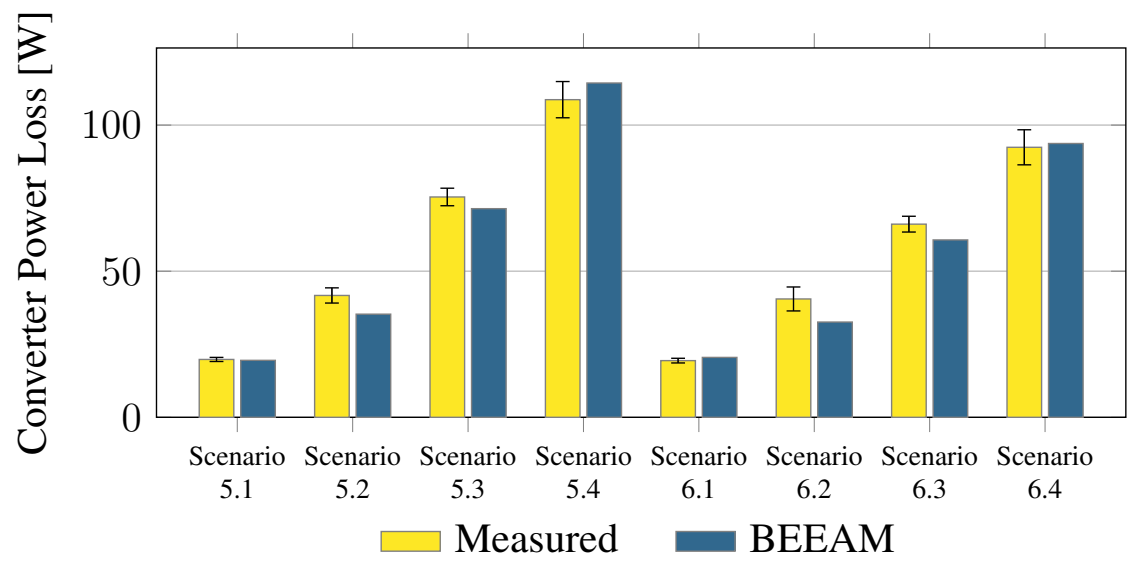

Figure C.12: Predicted versus measured converter loss, Scenario 5-6. 